\title{
WOMEN MIDLEVEL STUDENT AFFAIRS PRACTITIONERS: \\ EXPLORING THROUGH NARRATIVE INQUIRY THE SKILLS, ABILITIES, AND EXPERTISE NEEDED FOR CAREER SUCCESS
}

\author{
A Dissertation \\ presented to \\ the Faculty of the Graduate School \\ at the University of Missouri-Columbia \\ In Partial Fulfillment \\ of the Requirements for the Degree \\ Doctor of Philosophy \\ by \\ LAURA ALEXANDER PAGE \\ Dr. Casandra Harper Morris, Dissertation Supervisor \\ July 2017
}


The undersigned, appointed by the dean of the Graduate School, have examined the dissertation entitled

WOMEN MIDLEVEL STUDENT AFFAIRS PRACTITIONERS: EXPLORING THROUGH NARRATIVE INQUIRY THE SKILLS, ABILITIES, AND EXPERTISE NEEDED FOR CAREER SUCCESS

presented by Laura Alexander Page, a candidate for the degree of doctor of philosophy, and hereby certify that, in their opinion, it is worthy of acceptance.

Professor Casandra Harper Morris

Professor Jeni Hart

Dr. Cathy Scroggs

Professor Crystal Gateley 


\section{DEDICATION}

This dissertation is dedicated to my true partner in life and greatest encourager,

Dr. Tyler James Page, the most authentic person I know.

Also to my three brilliant, kind, and inspirational little women and self-proclaimed feminists,

Kate Elizabeth, Harper James, and Madeline Alexander, who call me Mama Ph.D. 


\section{ACKNOWLEDGEMENTS}

As I complete my Ph.D. journey, there are numerous individuals who deserve my sincere appreciation for their continuous support. To all who offered encouraging words, hugs, handwritten notes, scripture written on post-it notes, surprises on my front porch, coffee chats, and emails and texts that seemed to appear at just the perfect moment, I can never say thank you enough. This is indeed a degree in perseverance, and I owe my success to many.

I remain inspired by the four women on my committee. Dr. Casandra Harper Morris, my dissertation chair, I kept your e-mail confirming my acceptance into the program. Thank you for advising me through coursework and the dissertation; your editing skills are beyond impressive, and I appreciate you asking me challenging questions that pushed me to do my best work. You included me in work that resulted in my first publication, and together, we navigated co-teaching a course during an especially challenging campus climate.

Dr. Jeni Hart, you have become an important mentor in my life. I have personally benefitted from your expertise as a feminist scholar, your teaching and leadership in ELPA, your knowledge of higher education and student affairs, and your friendship. Your support of our family began as we started at Mizzou, through Tyler's doctoral journey, and mine. I am proud that our girls know and respect you. Our conversations lead me to consider new perspectives, and I always count on your honest insight. I look forward to our continued work together.

Dr. Cathy Scroggs, you took a chance in hiring me a decade ago when I literally showed up on your doorstep. You have always been open to my ideas for new initiatives, and our work together is a highlight of my career. Your leadership as a working mother who successfully 
navigated this profession is an example to me, but what I appreciate most is the advice you share in our candid conversations. I look forward to our coffee mornings and poolside chats.

Dr. Crystal Gateley, few people would have been receptive to an unknown student reaching out for dissertation assistance, but your enthusiastic welcome was just what I needed. Jeni knew what she was doing when she suggested us working together. Thank you for listening intently, asking important questions, and sharing your personal and professional experiences. Your successes as a Mama Ph.D. and a clinical faculty member remind me that we can do it all.

Dr. Jennifer Fellabaum-Toston, you are the consistent source of support woven through my doctoral story. You advised, supervised, challenged, and encouraged me every semester. Thank you for including me in opportunities that utilized my strengths, yet challenged me to expand my skillset. Our shared identity as practitioner-scholars has laid the foundation for many collaborations in the future.

Central to this dissertation are the six women who so graciously shared their stories. Your trust in me made this project possible, and I greatly appreciate your time and honesty. I have learned so much from each of you, and am inspired by your authenticity.

To the faculty and students of ELPA, it was a big decision to return to the classroom, but I have never looked back. I enjoyed our class discussions, sharing stories, and learning more than I ever anticipated. Dr. Sarah Diem, Dr. Brad Curs, Dr. James Sebastian, Dr. Lisa Dorner, Dr. Ty Douglas, Betty Kissane, Jude Sommerjones, Sara Cochran, Van Nguyen, and my "unofficial" cohort, I am a better scholar, teacher, professional, and leader because of the community we created.

To the PhD Prayer Group that emerged from our doctoral program - Rhodesia McMillian, Mike Kateman, Jason McKinney, Dena Lane-Bonds, Ransford Pinto, Jude Kyoore, 
and Kim Starks Berglund - your faith is inspiring and your prayers always authentic. I intend to remain part of our monthly gatherings. To Rho and Mike, special appreciation for pushing me to complete my dissertation, the writing days, and the texts. Let's cross the stage together.

To my Student Affairs colleagues at Clemson University, the University of South Carolina, the University of Missouri, and NASPA, thank you for inspiring me to do this work; I believe it is our calling. Special appreciation to Dr. Tony Cawthon, Dr. Pam Havice, Robert Barkley, Jerry Brewer, Dr. Dennis Pruitt, Dr. Jeanine Ward-Roof, and my NASPA IV-West family. There are too many others to list, but you all know who you are.

To my village - the friends who understand this process and have stuck by me from day one, Dr. Anna Edwards and Dr. Sara Hartley; Mizzou colleagues who became lifelong friends, Julie Drury, Ashli Grabau, Iris Calloway, Leslie Gale, Mollie Landers, Nicole Phillips, Michelle Marsden, Sean Joy, Shannon Breske, and Terri Gray; my fellow Southern transplants, Jac Jorgensen and Holly Elarbee; my Grant Elementary moms who checked it with me daily, Kari Gingrich, Dr. Claire Syler, and Lisa Stockman; our amazing small group from The Crossing; and true friends, Litsa Rivers, Bridget Kevin-Myers, Beth Stubbs, and Andrew and Jessica Bach. Many more I could list and whom I plan to thank in person.

To Stan and Jean, who welcomed me with open arms, thank you for placing a high value on education, for your encouragement during this process, and for driving down to spend time with the girls so that I could focus on writing. I am grateful to be a Page.

To my own sweet parents, Jimmy and Barbara, my biggest fans. You taught by example to put others first, and I am indebted to the sacrifices you made so that I could have so many opportunities. You have always believed in me, and I share this milestone with you. 
To Tyler, it is my greatest honor to share this life with you. It has been quite the successful decade - two Ph.Ds. and three amazing daughters. Few would embark on such a journey, but we kept the end goal in mind, and we made it. Our partnership is the central ingredient to the Team Page we have built, and I truly thank you for talking me through my ideas, being a solo parent while I finished papers, making my coffee and lunch, calming my stress, and reminding me that I am capable. One of us has been in school for the majority of our marriage. The hard work has paid off and I cannot wait to start the next chapter with you.

Oh, my sweet girls, Kate, Harper, and Madeline. Your sincere interest in my studies was always welcome, and I am amazed at how much you understand. It was difficult to drive back to campus for evening classes and see you standing on the porch, but I knew the fun Daddy had planned in my absence. You never complained; instead, you left drawings on my pillow, and in my class notes, and told your teachers and classmates of my progress. We always say that "girls can do anything" and I hope you will reflect on this time with pride, and that it serves as motivation as you work towards your own goals. YOU are my greatest achievement.

I thank God for giving me an inquisitive mind, the motivation to accomplish my life goals, and an unwavering support system. Philippians 1:6 And I am sure of this, that he who began a good work in you will bring it to completion at the day of Jesus Christ. May this part of my journey, and everything I do moving forward, glorify Him. 


\section{TABLE OF CONTENTS}

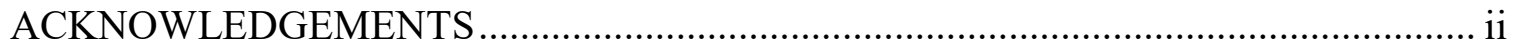

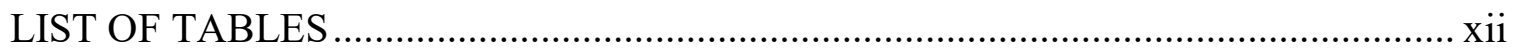

LIST OF FIGURES ….................................................................................. xiii

\section{Chapters}

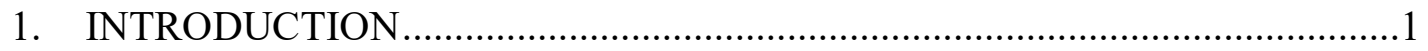

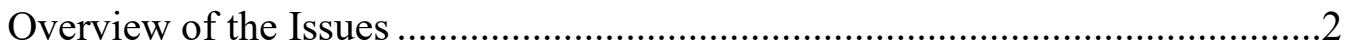

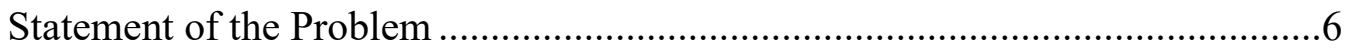

Purpose of the Study and Research Questions .............................................

Overview of the Theoretical Framework ....................................................... 9

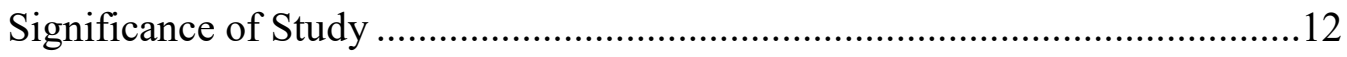

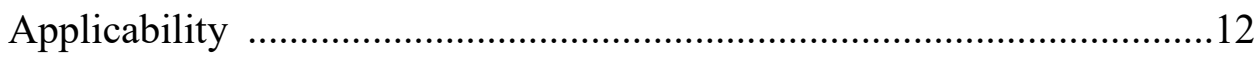

Methodological choice ...............................................................13

Power of the meta-narrative ........................................................... 13

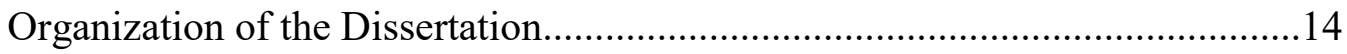

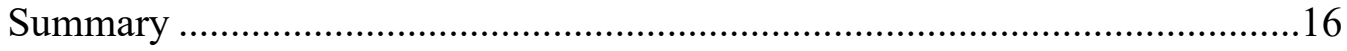

2. REVIEW OF THE LITERATURE .......................................................... 18

The Student Affairs Profession ................................................................. 18

Knowledge acquisition and investment ............................................20

Involvement f.......................................................................... 21

Competence and Stages of Career Development ........................................22

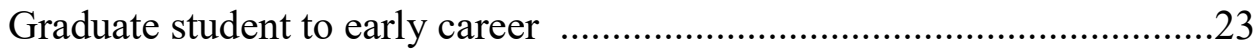

Insights from midlevel and SSAO professionals ...............................24

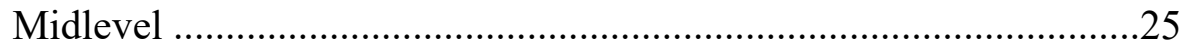

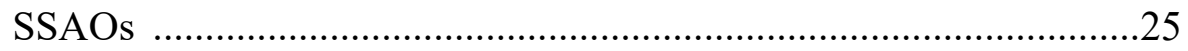


Professional competency areas for student affairs educators

Creation 26

Implementation .................................................................29

Women Midlevel Student Affairs Practitioners .............................................30

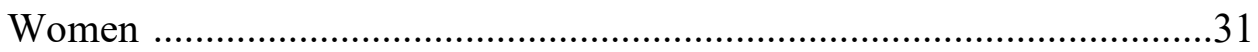

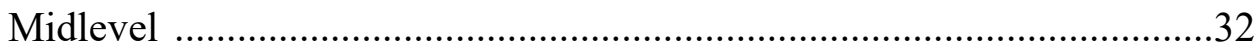

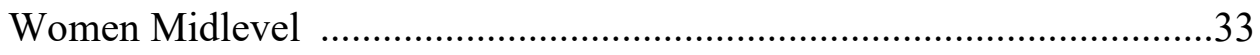

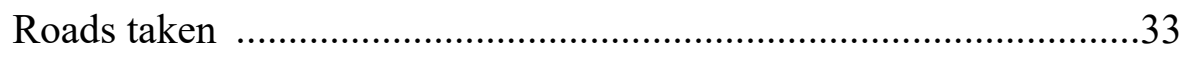

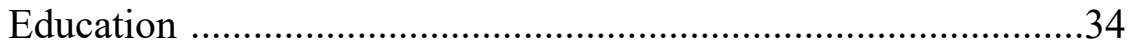

Personal and professional life balance ....................................34

Career development ......................................................... 35

Importance of additional research .............................................36

Overview of the Theoretical Framework: The Kaleidoscope Career Model ....37

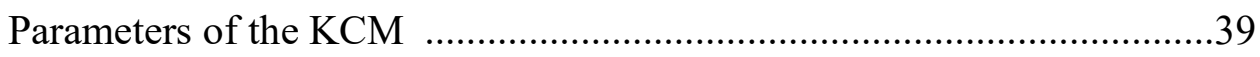

Applying the $\mathrm{KCM}$ to research ........................................................41

KCM and student affairs .........................................................4

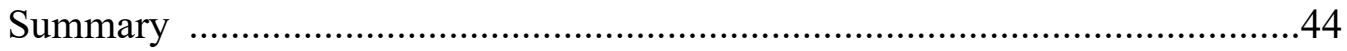

3. METHODOLOGY AND RESEARCH DESIGN ......................................45

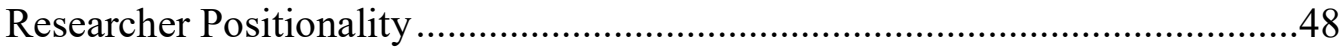

Participants and Sampling Procedure ......................................................50 


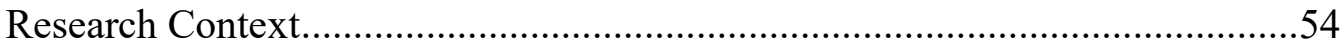

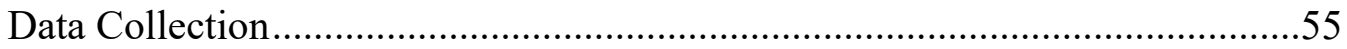

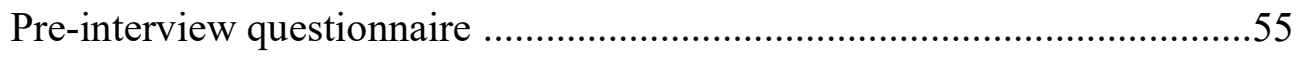

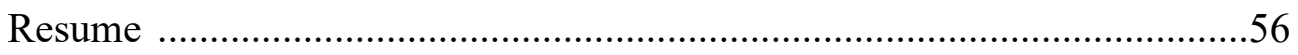

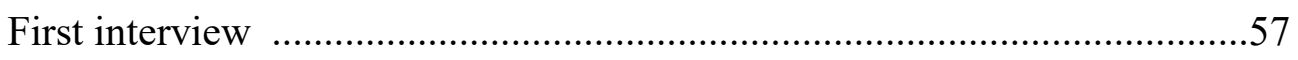

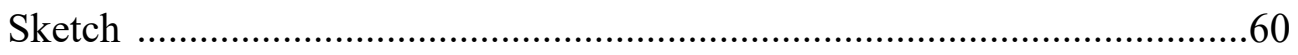

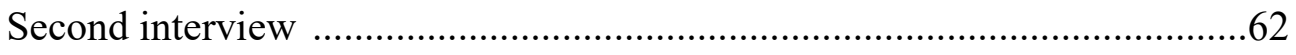

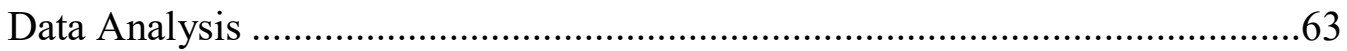

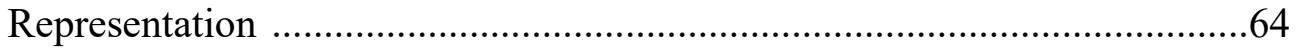

Demographic and academic background information .............................65

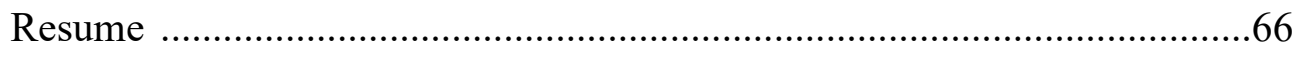

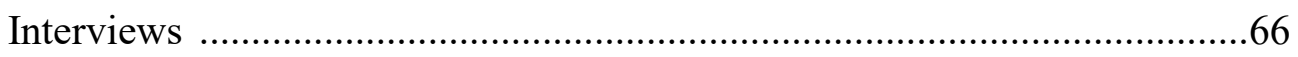

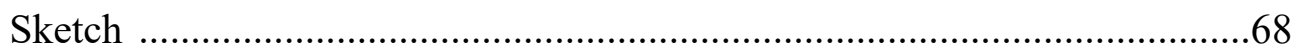

Thematic narrative analysis .............................................................6

Use of Theoretical Framework in the Research Process ..................................70

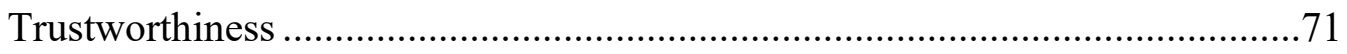

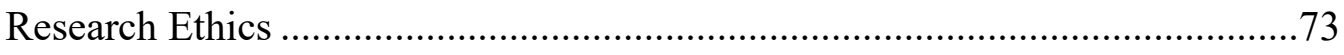

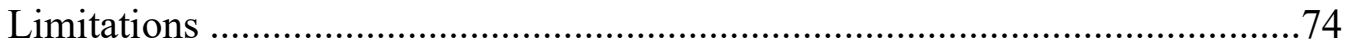

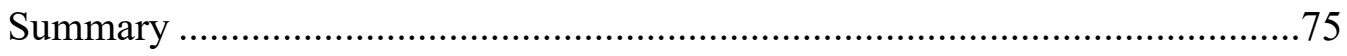

4. PRESENTATION OF INDIVIDUAL NARRATIVES ................................77

Differences and Commonalities ........................................................ 78

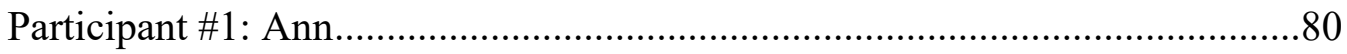




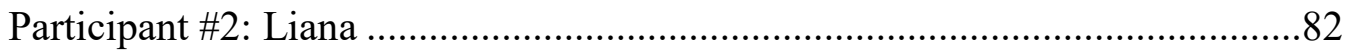

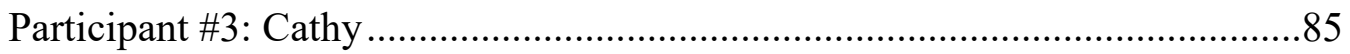

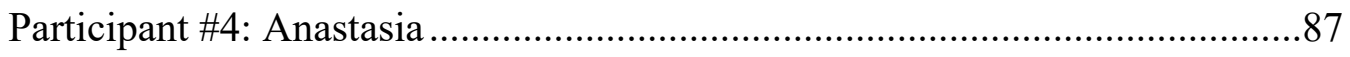

Participant \#5: Betty ........................................................................ 90

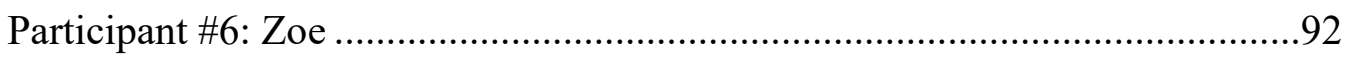

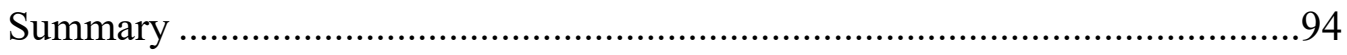

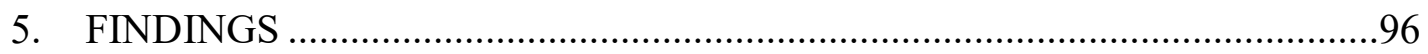

Theme 1: Reluctance to take full credit for career success .............................98

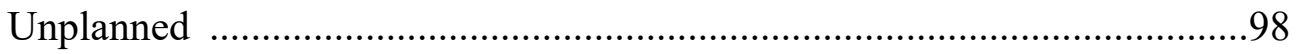

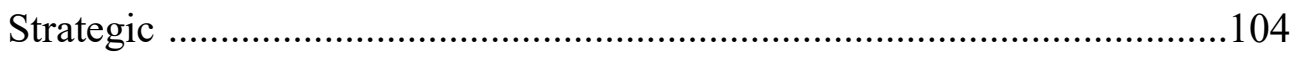

Theme 2: Defining success through feelings ............................................109

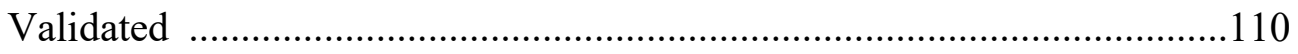

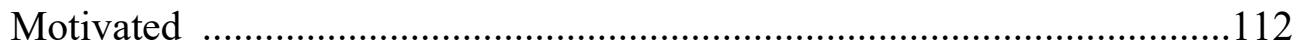

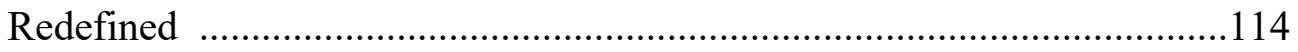

Theme 3: Misalignment between supervisor philosophy and actions.............115

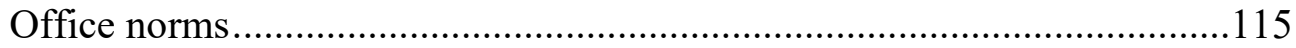

Impact on supervisory style ...................................................... 121

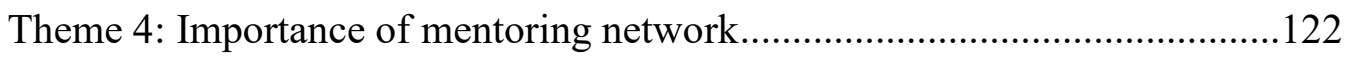

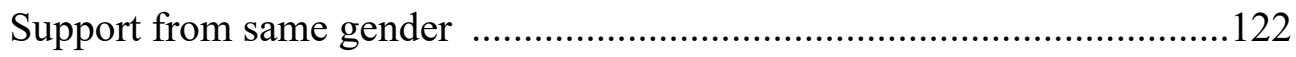

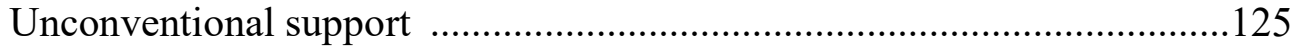

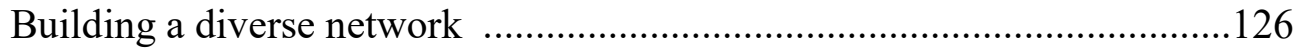

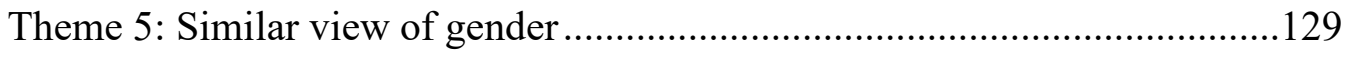

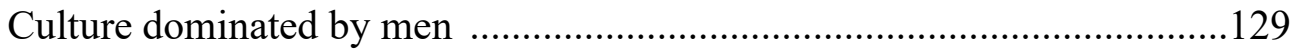

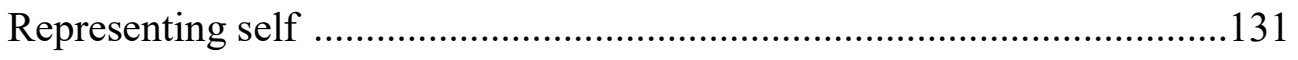

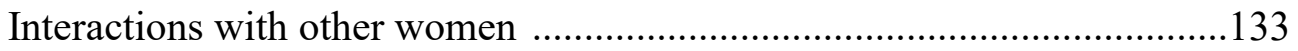


Gender as a strength

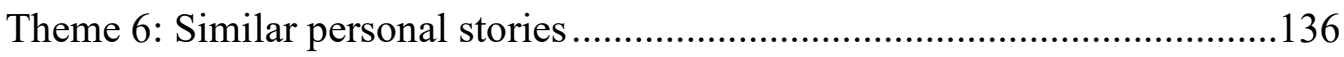

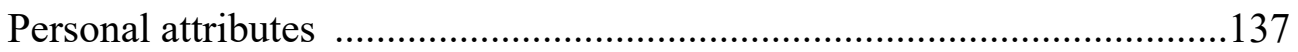

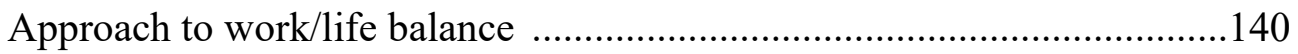

Approach to professional development ................................................ 143

Theme 7: Advice for the future of the field ..............................................144

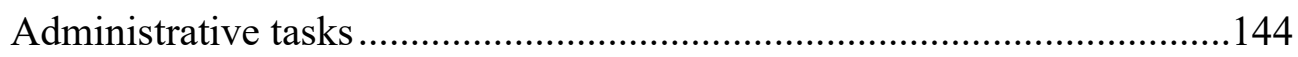

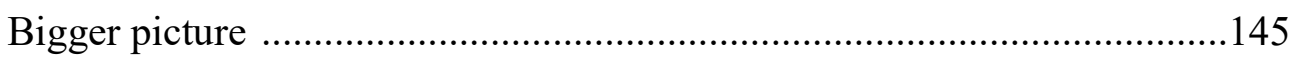

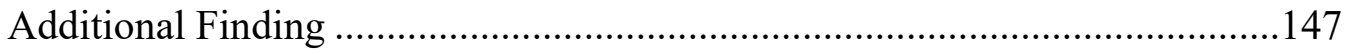

Sharing their personal narrative included emotion $\ldots \ldots \ldots \ldots \ldots \ldots \ldots \ldots 147$

Interpretation of Themes through Theoretical Framework ...........................148

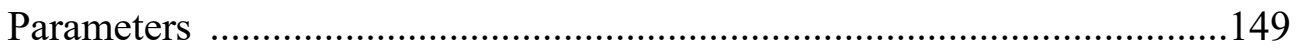

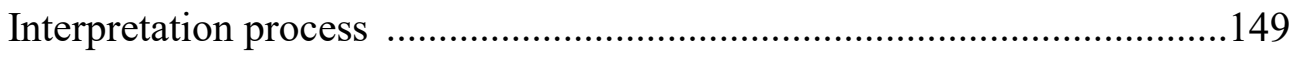

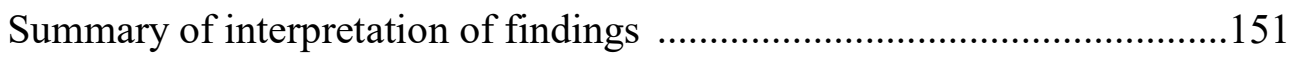

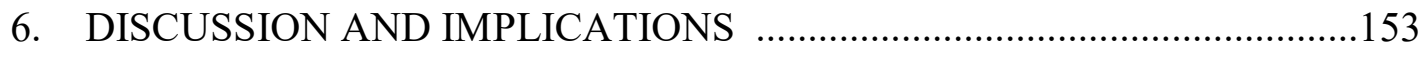

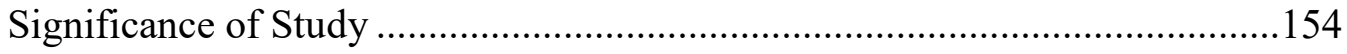

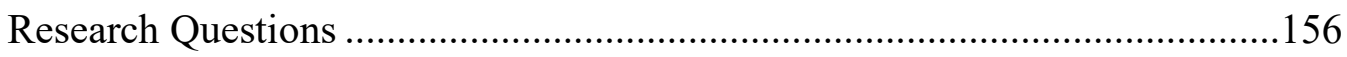

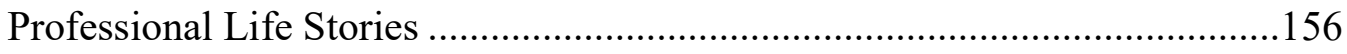

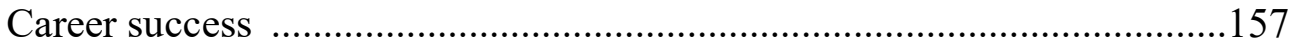

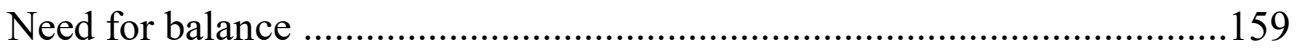

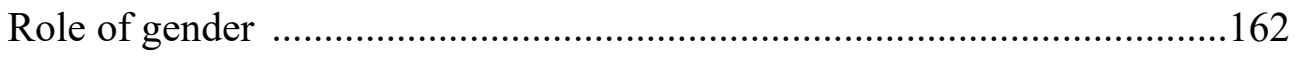

Conceptualization of Competency Development........................................164

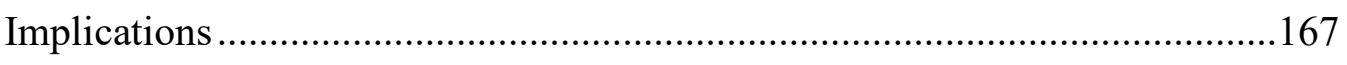

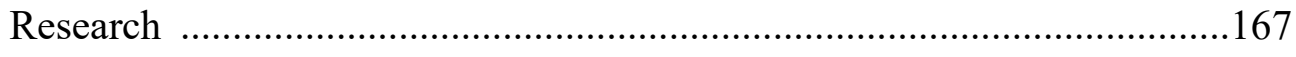

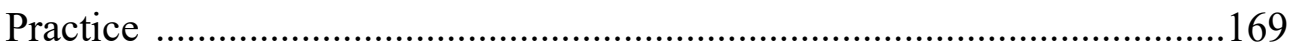


APPENDIX
A. E-MAIL INVITATION PROTOCOL

B. PREPARING FOR FIRST INTERVIEW

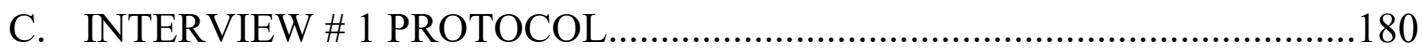

D. MEMBER CHECKING \& SECOND INTERVIEW SCHEDULING .............181

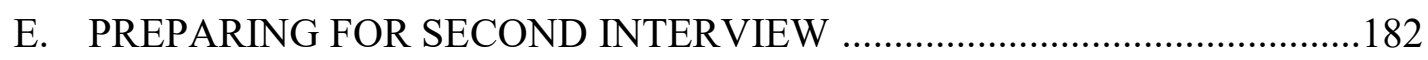

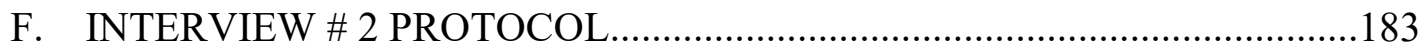

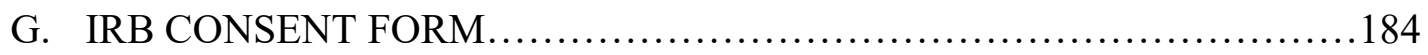

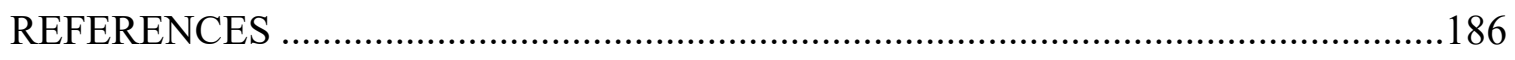

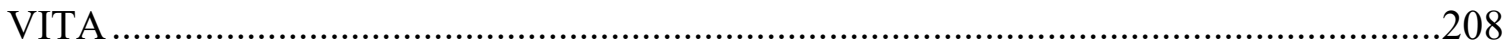




\section{LIST OF TABLES}

Table

Page

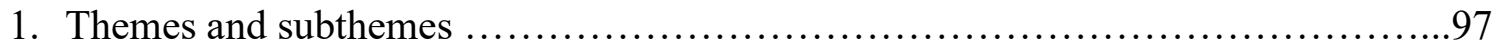

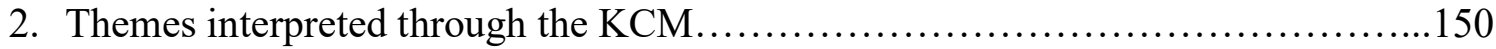




\section{LIST OF FIGURES}

Figure $\quad$ Page

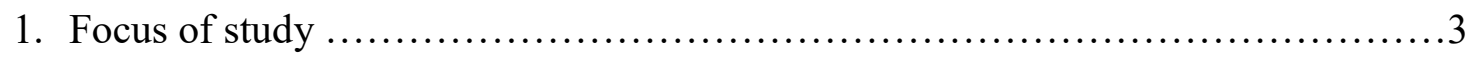

2. Unplanned career turns from Cathy's sketch ................................................100

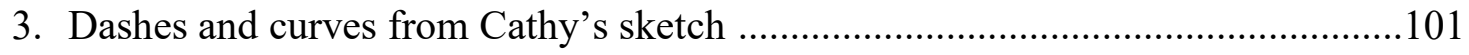

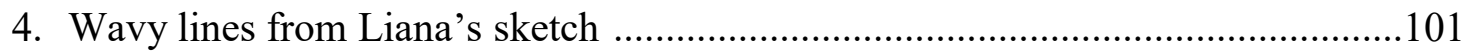

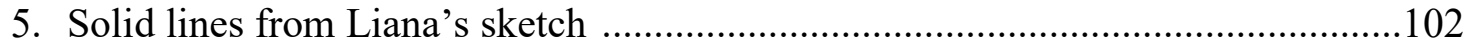

6. Detour from Anastasia's sketch ........................................................................... 103

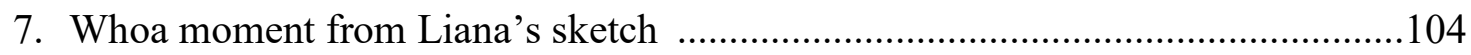

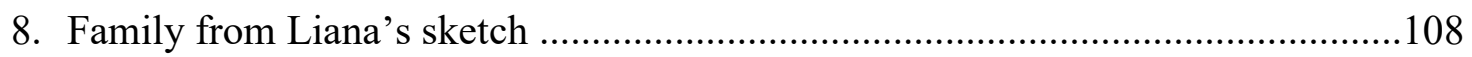

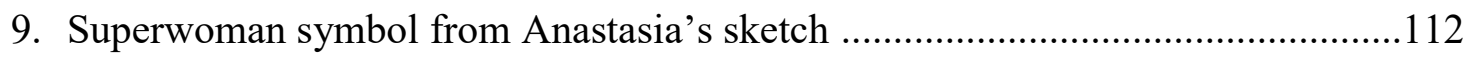

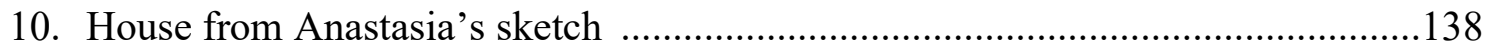

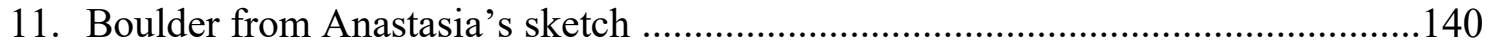




\section{Chapter 1: Introduction}

What contributes to a successful career in the student affairs field, particularly for women at the midpoint? What can be learned from understanding an individual's professional story and how they describe the opportunities and challenges, personal decisions, advancements and setbacks, and influences of gender that contribute to where their story has led them? Since little research exists on the careers of women midlevel practitioners, we are unable to determine what contributes to a successful career. Such questions serve as the motivation for this study.

As a higher education professional for over 15 years, I embrace the identities of woman and midlevel regarding my roles as a student affairs practitioner and scholar. I have built an extensive network of colleagues from colleges and universities across the United States, and internationally, and we communicate frequently about the challenges associated with career advancement. Specifically, we contemplate the competencies needed for career success and how they may be obtained, methods for navigating the field as a woman, and our attempts to devote necessary attention to our professional and personal life. Adding family responsibilities to the latter item makes the situation more difficult as we work to redefine what it means to be successful.

Since success looks different for everyone, it is important to pay attention to variances between career stories. However, the field of student affairs is collaborative, where sharing insight and best practices is common. Therefore, collecting individual stories to build a metanarrative is conducive to better supporting this population. Furthermore, this research advances the field's understanding of career development and how the field conceptualizes the competency development associated with career advancement and success. 
This study explores the professional stories of women midlevel student affairs practitioners. Through narrative inquiry, participants share their professional life stories, including details regarding what skills, experiences, abilities, and expertise they believe have contributed to their individual career development, as well as the role gender has played. Using the Kaleidoscope Career Model (KCM) (Mainiero \& Sullivan, 2005, 2006) as a guiding framework for the study, themes were identified from the findings and then interpreted through the KCM's components of authenticity, balance, and challenge. Findings from this study serve as the impetus for subsequent studies focused on exploring individual career development, competency development, and gender. Practical implications from this study reveal key aspects of professional development applicable to all aspects of the student affairs profession, including individual practitioners, departments and divisions at higher education institutions, and the professional associations that provide professional development and guidance to the field.

\section{Overview of the Issues}

A recent poll (Jones, 2016) revealed that $70 \%$ of Americans believe postsecondary education is important, and the National Center for Education Statistics (2016) reported that over 7,000 postsecondary institutions currently exist in our country. Within these institutions, the field of student affairs is large and diverse with a long history of supporting students as they pursue a college degree. Student affairs staff work in a multitude of student service roles on every campus. To give a sense of the size of the field, the United States Department of Labor reported in 2014 that over 175,000 postsecondary education administrators, the most closely related title that would include student affairs staff, were employed by United States institutions of higher education. The significant number of staff working in student affairs roles merits further study of the various aspects of these careers. 
This study began with a broad interest in learning more about the various aspects of career development, particularly the ingredients needed for success. Since no singular definition exists for the term success, the study relies heavily on two sets of interpretations of what it means to be successful; first, from the perspective of the author in my selection of participants, and second, from the perspectives of the women who shared their narratives. Given the numerous directions one could take in studying this topic, it was imperative to narrow the focus. Figure 1 provides a visual depiction of the aspects of career development explored, the population to be examined, the methodological choice, and goals for using the findings to inform the field of student affairs.

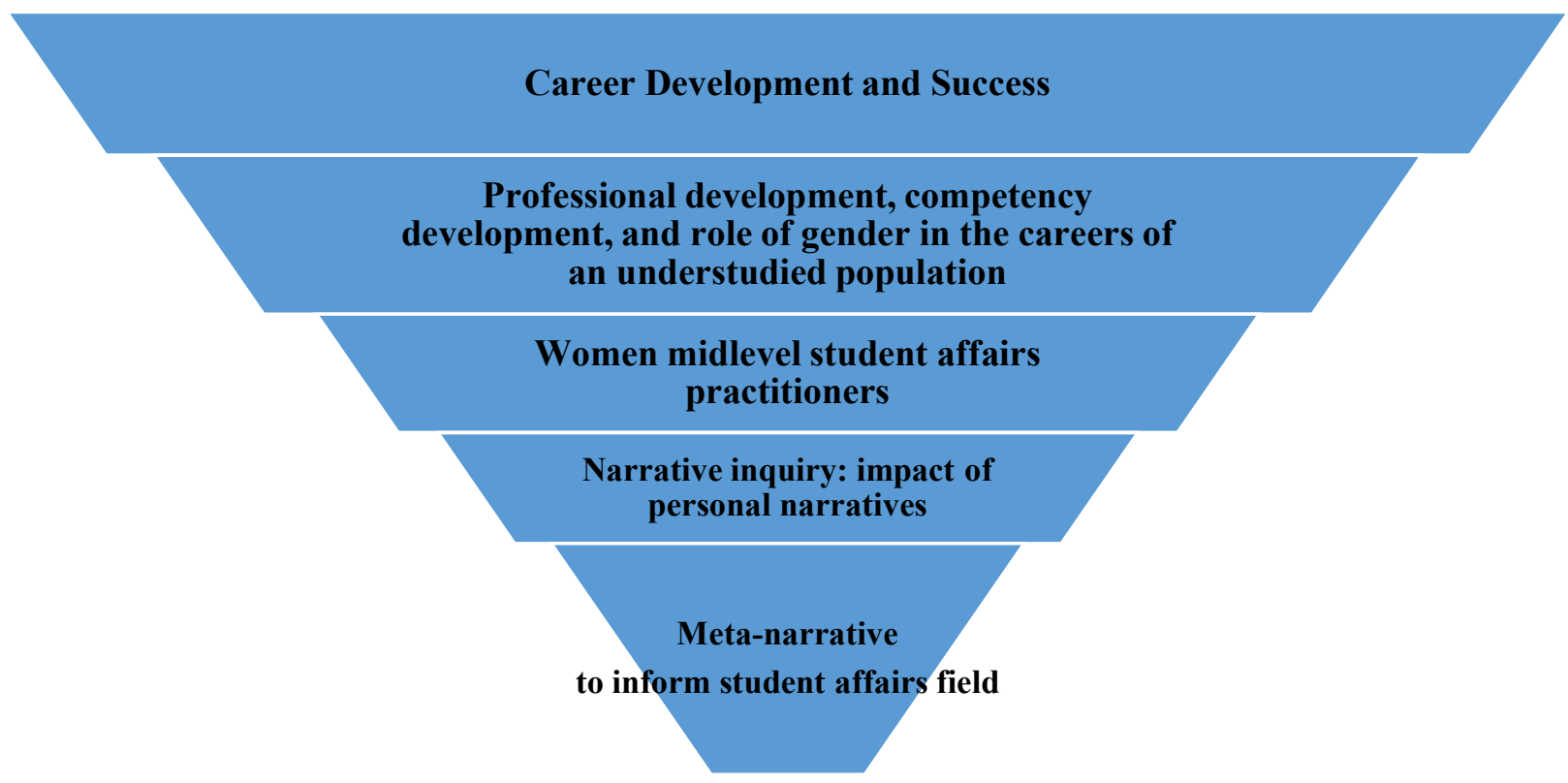

Figure 1. Focus of study

Through each step of the research process, referencing this depiction of the focus of the study ensured the project remained on track to accomplish its goals.

Starting with the broader interest areas of this study is a look at career development and success, specifically professional development and competency development. The educational community is consistently exploring innovative approaches for involving individuals in their 
own professional development (Burbank \& Kauchak, 2003). Included in these efforts within higher education, the field of student affairs has long prided itself in developing practitioners, and there exists a linear structure in opportunities for career advancement. Current discourse in student affairs regarding career development on both the campus level, and within national professional associations, has focused primarily on the idea of competencies, specifically guided by the two leading professional associations for the field, College Student Educators International (ACPA) and Student Affairs Administrators in Higher Education (NASPA). However, the overall concept of competence attainment is broad. Additionally, the role competency development plays within a student affairs career likely varies greatly between individual practitioners based on factors such as educational background, job responsibilities, culture of the institution where they are employed, and both short-term and long-term career goals.

While the competency discourse in student affairs has recently experienced renewed focus in the literature (Eaton, 2016; Gansemer-Topf \& Ryder, 2017; Herdlein, Riefler, \& Mrowka, 2013) and conversations amongst practitioners, little empirical evidence exists to understand the competency and career development phenomenon. For example, within the field of student affairs, questions remain regarding the utility of the Professional Competency Areas for Student Affairs Educators (ACPA/NASPA, 2015). Although there appears to be general interest in the competencies and buy-in by practitioners, there is scant empiric evidence related to the creation of the competencies. The competency document was created by a task force of members from the two largest student affairs professional associations: ACPA and NASPA. The process did provide multiple opportunities for feedback from association members 
(ACPA/NASPA, 2015), but clear recommendations for the implementation, use, and benefit of incorporating the competencies into professional practice have yet to be released.

Regarding perspectives on individual career development in student affairs, some research exists on the mid-manager position in student affairs, although this group is typically referred to as midlevel in the field. Though a formal definition for midlevel student affairs does not exist, the term is typically understood as the space between entry-level and senior leadership (Ackerman, 2007). Another classification is that midlevel usually report directly to the Senior Student Affairs Officer (SSAO), hold managerial and/or administrative roles, and oversee programs and departments within a larger division of student affairs (Rosser \& Javinar, 2003). Some midlevel professionals aspire to advancing to a senior administrator position, while others will remain midlevel but may be entrusted with additional responsibilities in their current position or may transition to other units within student affairs departments and divisions. Regardless of their career goals, the midlevel career point is an interesting juncture, as these staff members are no longer new professionals, yet they are not perceived as top administrators. As the literature review reveals, many studies exploring job performance, job satisfaction, and career success focus either on entry-level professionals or Chief Student Affairs Officers (e.g., deans, vice presidents).

Adding another layer to this study is the intentional selection of women midlevel practitioners. I acknowledge referring to gender within broad identity categories may be limited and exclusionary (Butler, 1993). I sought out women-identified individuals for this study rather than exploring more fluid gender categories (e.g., transgender, gender non-binary, gender nonconforming). All participants were given an opportunity to indicate their gender identity, and all indicated woman as their choice. Including this aspect of their identity supports this study's 
attempt to better understand the impact of identifying as a woman in student affairs career development.

\section{Statement of the Problem}

Absent from the history of the competency discussion is the how in terms of competency attainment and its impact on career development. It is vital to explore the ways practitioners already working in the field develop (and have developed) competencies that have contributed to their professional development, professional mobility, and career success. Rather than a narrow focus on specific competencies, particularly the ACPA/NASPA Professional Competency Areas, more examination is needed on how student affairs careers develop to the point where practitioners believe they are successful. This study seeks to explore the experiences of a specific group of student affairs professionals with two identifying characteristics, gender identity (specifically identifying as a woman) and career stage.

Including gender and professional level as focus areas of this study is important for several reasons, including the lack of literature focused on women at midcareer and the increasing number of women who have reached top student affairs leadership positions in recent years. In 2000, Rosser asserted that midlevel professionals comprised the largest administrative group in most college and university systems. Based on the current NASPA membership database, the number of individuals who have self-reported both professional level and gender shows is 2,751 women midlevel professionals (J. Taylor, personal communication, June 29, 2017). There are some aspects of the student affairs career that suggest a linear career path, such as professional categories named new professional, midlevel, and senior. However, anecdotal stories about practitioners who have reached the top positions through a variety of pathways. Duffy (2010) noted that more women are securing top leadership positions, which indicates some 
progress; however, gender equity has not been achieved among student affairs administrators. A 2014 census survey by NASPA garnered feedback from a third of institutions in the U.S. and showed $47 \%$ of vice presidents for student affairs are women. While the percentage suggests gender equity in SSAOs, it is pertinent to note that each institution typically has just one individual who holds the SSAO or Dean of Students title, and they report directly to university presidents (Bass, 2006; Kuk \& Banning, 2016). With many women at midlevel and so few top positions available, women seem to be clustered at the middle of the organizational chart. If the end goal for some women working in student affairs is to become an SSAO, then studying women who have chosen to stay in the field will help us know more about competencies, how they develop, and how they might continue to develop; all important to our understanding of career advancement. However, this may not be the preferred career path for all women, and some may choose to remain at midlevel.

Aside from their end career goals, discussion related to professionals who work in the student affairs field tends to focus more on supporting individuals at the start of their professional journey or seeking advice from those who achieved the most senior positions in the field (Ackerman, 2007). Less has been directed at midlevel practitioners. Thus, combining the focus areas of gender (i.e., women) and career stage (i.e, midlevel) identities results in few studies. Reviewing the limited existing literature on these topics supports the justification for a study on women, midlevel practitioners to fill an obvious void.

\section{Purpose of the Study and Research Questions}

The purpose of this qualitative study is twofold: first, to use narrative inquiry and the parameters of the KCM to explore how women midlevel student affairs practitioners have developed the competencies needed for career success, paying attention to the role of gender in 
their career development; and secondly, to add to the research on career development in the field of student affairs by focusing on a specific understudied population. This study attempts to fill a gap in the literature by taking a new direction in the research of professional competency in student affairs. As mentioned, attempts have been made for many years to identify the competencies necessary to succeed as a student affairs professional. With the release of The Professional Competency Areas for Student Affairs Educators (ACPA/NASPA, 2015) conversations at annual conferences and on individual campuses have tended to focus on best practices in using the framework. But little focus has been directed on the ways in which individuals obtain competence. The purpose of this qualitative study is to explore how a specific group of professionals has developed the competencies needed for career success, and to explore the role of gender in their career development.

Guided by the KCM as the theoretical framework and narrative inquiry as the method, I conducted interviews with women midlevel student affairs practitioners from different institutions in the Midwest region of the United States. Information collected was coded for themes, which were later interpreted through the KCM's parameters of authenticity, balance, and challenge. The two-step analysis allowed me to better comprehend the various methods of competency development in the career development of this population and guided the discussion section.

Findings from this study provide insight into how competence is viewed and achieved from the perspective of women midlevel professionals, as well as further our understanding of gender through the career development process in student affairs. The following research questions were addressed: 
1. How do women midlevel student affairs practitioners describe their professional life story, particularly their current levels of success within the field?

a. To what do they attribute that success?

b. What role has gender played?

2. What do their histories reveal about key aspects of their personal attributes, skill development, and professional experiences that might help inform the field's conceptualization of competency development?

\section{Overview of the Theoretical Framework}

In search of a framework that was relevant to the research questions and the problem this study seeks to address, I explored several grounded in the education discipline. While several had the potential to relate to portions of my overall study, it took time to identify a model strong enough to guide the study from start to finish, influence data collection, and serve an important part of data analysis.

When I initially designed the plan for this study, I was most interested in comprehending the various methods of competency development. Thus, I sought to use both a conceptual and a theoretical framework. The Professional Competency Areas for Student Affairs Practitioners (ACPA/NASPA, 2015) were considered as a conceptual framework for the study, and for their specific use in the data collection phase to examine the professional development of student affairs professionals. Rounding out my complex plan of using two frameworks, I considered Kolb's Experiential Learning Cycle (1984) as the theoretical framework for the study, specifically to guide the data analysis. I planned to interpret data and categorize findings using the four headings of the Kolb's cycle: concrete experience, reflective observation, abstract conceptualism, and active experimentation. As I struggled to make all the pieces of my study 
connect to these frameworks, two revelations occurred. First, delving more into Kolb's work revealed criticisms of the learning cycle that led me to question its relevance to my project. Secondly, I became less focused on examining only the process of competency development, and increasingly more interested in which population I wanted to study.

I reflected more on my own experiences as a woman student affairs practitioner who had reached the midlevel point of a student affairs career. This process underscored the importance of gender and professional level as integral pieces of my study. Still interested in competency development, my focus population directed my next exploration of potential frameworks. In terms of the role women play in their own competency development, self-authorship (BaxterMagolda, 1992) provided a valid connection between its concepts of reflection in goal setting and intentional planning and my view of competency development. However, most of the work around self-authorship centers on undergraduate student success (Perez, 2014), and given the scope of the problems I hoped to address, the framework seemed limiting. Still interested in the role one plays in developing themselves professionally, I briefly explored self-efficacy theory (Bandura, 1977). While the theory fit well based on the belief in one's ability to influence events, I determined that it placed too much emphasis on what the individual can control, thus would also be limiting to the purpose of my study.

Eventually, I also did not select the next option as the framework; however, it served influential in motivating me to step back and examine the purpose of my study from a larger perspective. Based on Bandura's social cognitive theory, Social Cognitive Career Theory or SCCT (Lent, Brown, \& Hackett, 1994) revealed the interrelated complexities that exist in career development. Now able to conceptualize a way to examine competency development within a 
larger context of career development, and keeping the population of interest at the forefront, led me to explore additional frameworks within the career context.

The Kaleidoscope Career Model (KCM) created by Mainiero and Sullivan $(2005 ; 2006)$ uses the artistic metaphor of a kaleidoscope to describe the many correlating decisions that impact a woman's career. Pertinent to this study, the model recognizes the complexities of individual career development. Specifically, the KCM illustrates how one decision simultaneously affects multiple aspects of one's life. The three parameters of the KCM authenticity, balance, and challenge - clearly connect to the multiple interest areas of this study in that they enable me to: (a) attend to the importance of the individual, as well as the importance of gender; (b) acknowledge that career decisions are made with many factors in mind; (c) remember that competency development is a combination of what is inherently part of a person as well as what is learned; (d) consider the impact of women sharing their own stories as a way to impact the field.

Although the KCM may be rooted in business, its potential applicability to working women in all fields makes it an attractive model to frame the study of women midlevel student affairs practitioners. In fact, the concept of using a model created outside of the field of higher education brings a new perspective to the examination, leading to unique findings. As displayed in their work on leadership, Kezar, Carducci, and Contreras-McGavin (2006) articulated the use of outside theories as beneficial in helping professionals understand complex issues and advocate for more adaptive systems and practices. Thus, an intentional selection of a framework from a discipline outside of higher education brings a fresh perspective to understanding the longdiscussed conversation on competencies and career success in student affairs. I go into more detail about the KCM in chapters 2 and 3. 


\section{Significance of Study}

The timing and applicability of contributing new information to the competencies discourse show relevance for the study. The qualitative methodological choice of narrative inquiry best explores the research question in ways that reach the participants at a level that enables the sharing of information integral to our understanding of these issues. Using my professional background as a woman midlevel student affairs practitioner as a foundation, and interest in bridging theory to practice, findings from the study have strong implications for research, practice, and theory.

\section{Applicability}

The findings of this study are pertinent to the larger discourse within the field regarding competencies, and have applicability at the individual level. Hoffman and Bresciani (2012) concluded their study on student affairs competencies with this statement, which is both relevant and encouraging for this study:

Ongoing research and scholarly discourse regarding the scope and content of competencies will continue to be critical as the student affairs profession intentionally designs and implements professional preparation programs and professional development to educate the people who work so diligently to promote access, equity, and overall student success within higher education. (p. 38)

Not only do the findings from this study further the field's understanding of competency development, they produced several implications for research, theory, and practice discussed in Chapter 6. Among the suggestions is an emphasis on the need for applicability of the competencies to ensure their usefulness within professional development. 


\section{Methodological Choice}

A fundamental aspect of this study is the methodological choice. Qualitative research is useful when we want to empower individuals to share their stories (Clandinin \& Connelly, 2000; Creswell, 2013; Riessman, 2008). At the onset of this research, I determined the best way to learn more about this population is to ask them to share. The intentional choice of narrative inquiry to explore these issues enhances the significance of the study for many reasons, primarily that there is much to be learned from individual career paths, as told by the practitioners themselves, and to learn from the lived experiences of others. Beneficial insight can be gleaned from women midlevel practitioners sharing their stories in their own voice. In fact, telling stories about past events appears to be a universal activity amongst all humans, and as children, one of the first forms of discourse one learns (Nelson, 1989). The practice of storytelling continues throughout life by people of all social backgrounds in a wide array of settings (Riessman, 1993).

My acknowledgement that fundamental differences exist in how individuals approach career development further substantiates the need to examine individual stories. Spence (1982) distinguishes between historical and narrative truth, with the latter a construction, non-factual version of the experiences. With respect for historical truths, this study takes the perspective that non-factual accounts provide unique insight with ability to impact others. Rather than attempting to generalize, narrative inquiry allowed me to carefully explore the multiple layers of the participants' stories (Clandinin \& Connelly, 2000) to make meaning of them separately, and together as a meta-narrative.

\section{Power of meta-narrative}

Within qualitative research, narrative inquiry is "both a project and a method" (Creswell, 2013), and the tenets of narrative inquiry permeated every step of this study — from the selection 
of participants, to the rapport built between myself and the women, to the interpretation of information shared. This study draws upon the experiences of a sample of practitioners who have displayed success in their careers to build a meta-narrative with potential to benefit practitioners at various levels of their own careers. It was essential that this study "capture the lived experience of people in terms of their own meaning making and to theorize about it in insightful ways" (Josselson, 2011, p. 225).

Identifying themes across the stories of multiple women can be informative to other practitioners who are navigating their own knowledge acquisition, practical experiences, and individual development plans. Competencies play an important role in the current student affairs discourse (Hoffman \& Bresciani, 2012). A desire to assist practitioners at various levels and stages of their careers in both their personal and professional development supports the need for this study. As such, this project seeks to empirically examine the factors these women believe have contributed to their career success, and how they understand the influence of their gender. A motivation for the study is to share the findings with the student affairs community through publication in higher education and student affairs journals, as well as presentations at the ACPA and NASPA annual conferences.

\section{Organization of the Dissertation}

In the following chapter, I present the literature review, which is divided into four sections. First, I begin with an overview of the student affairs profession with specific discussion on knowledge acquisition and investment, as well as involvement. Secondly, I explore the various perspectives of competence from stages of career development in student affairsgraduate students to early career and insights from midlevel and SSAO professionals. Within this section, I also provide a comprehensive overview of the professional competency areas for 
student affairs educators, including creation and implementation. Next, regarding the specific population for this study, I focus on both midlevel practitioners and women practitioners, and finally, the limited literature on women midlevel practitioners in student affairs. Within this section, I do acknowledge the influence of a key piece of literature on our understanding of this population. Finally, I transition to an in-depth overview of the framework that guided this study, the Kaleidoscope Career Model (KCM), including a synthesis of other studies that have used the framework.

In Chapter 3, I start with an explanation of narrative inquiry as the methodological choice for this qualitative study, as well as the research design. Next, I include a statement of my researcher positionality, pertinent to not only the purpose of the study but an integral part of my selection of participants. What follows is a description of the research context, and the study's participants and sampling procedure. Within my description of data collection is specific information related to the pre-interview questionnaire, collection of participant resumes, first interview, sketch activity, and second interview. I begin the data analysis explanation with a discussion of how representation in the research process, introduced by Riessman (1993), is important to the study, followed by a description of how each piece of data was analyzed through thematic narrative analysis. Then, I reveal how the $\mathrm{KCM}$ was used in the research process, as well as an overview of the strategies employed to ensure trustworthiness and research ethics. I also address the limitations of this study, before ending with a summary that supports the selection and use of narrative inquiry in this study.

Chapter 4 introduces the six women who shared their personal narratives through participation in this study - Ann, Liana, Cathy, Anastasia, Betty, and Zoe. I begin by addressing the differences and commonalities among their professional stories. Next, I provide a 
comprehensive overview of each individual participants. I end with a summary of my reflections of their stories, serving as a transition to the actual findings presented in Chapter 5.

In Chapter 5, I introduce findings that emerged from the data analysis of the preinterview questionnaire, resumes, sketch activity, and interview transcripts. A total of eight themes are explained, many with sub-themes. In addition, the chapter includes several images from the participants' sketch activity to provide a visual representation of some of the ways they described their professional stories. The chapter ends with an interpretation of the findings through the KCM, specifically the framework's parameters of authenticity, balance, and challenge.

In Chapter 6, I discuss the significance of the study in adhering to its purpose, which is to (a) use narrative inquiry and the parameters of the KCM to explore how women midlevel student affairs practitioners have developed the competencies needed for career success, paying attention to the role of gender in their career development; and (b) to add to the research on career development in the field of student affairs by focusing on a specific understudied population. Next, I address how the findings of this study help to answer the research questions through the lens of the KCM. I conclude with implications for research, practice, and theory.

\section{Summary}

Contributing to the current discourse on competency development in the field of student affairs is timely due to the release of formal competencies, as well as the ongoing focus of professional development of practitioners. Although the literature reveals various perspectives on which competencies are necessary to be successful in the field, less research has focused on the population of women midlevel student affairs practitioner. Since the experiences of new professionals and Chief Student Affairs Officers (CSAOs), which comprise the majority of the 
scholarship about the profession, do not reflect the uniqueness of the career midpoint, it is essential to explore the lived experiences of individual practitioners currently working in the field. Due to the ambiguous nature of how the Professional Competency Areas for Student Affairs Educators were created, as well as their intention for practical application, this study will revisit them in the discussion (Chapter 6). In the absence of empirically based competencies, this study provides a rich perspective on what women midlevel student affairs practitioners believe has contributed to their success, including the role of gender in their professional story. There is much to be gained from the findings of this study for those who research, practice, and theory in higher education and student affairs, as well as individuals seeking to pursue a career in the field. The following chapter offers a comprehensive literature review as a foundation to the study. 


\section{Chapter 2: Review of the Literature}

In this chapter, I review the literature related to this study. Specifically, I organize the chapter into the following sections: (1) the student affairs profession, (2) competence in student affairs, (3) women midlevel student affairs practitioners, and (4) the conceptual framework that guides this study.

\section{The Student Affairs Profession}

The student affairs profession has existed as far back as 1937 when the American Council for Education (ACE) formalized student affairs with the release of their report titled the Student Personnel Point of View (SPPV) (Nuss, 2003). The SPPV encouraged development of the field, including a national presence to assist efforts (ACE, 1937) and an emphasis on professional development (ACE, 1949). Student affairs has since experienced growth in all aspects of its services and functions within institutions of higher education in the United States. Scholarly publications continue to serve as avenues to share best practices and discuss the evolution of the field (Carpenter \& Haber-Curran, 2013).

As the field matured, two specific publications were integral in their encouragement of discussion and debate about the purpose of the field among its practitioners. The Student Learning Imperative (SLI) was a charge to practitioners to enhance the student experience by creating conditions that motivate students to create and participate in educationally-purposeful activities, inside and outside of the classroom (ACPA, 1996). Continuing with student affairs' commitment to holistic development of students, Learning Reconsidered (Keeling, 2004) was a joint publication by ACPA and NASPA that addressed the changing profile of the American college student by arguing for the "integrated use of all higher education's resources in the education and preparation of the whole student" (p. 3). A second version of Learning Reconsidered (Keeling, 2006) incorporated additional professional associations as collaborators, 
the Association of College \& University Housing Officers International (ACHUO-I), the National Academic Advising Association (NACADA), and the National Association of College Unions International (ACUI) to name a few. These collaboration efforts advanced conversations in the field regarding a campus-wide focus on the student experience, offering practical recommendations. Subsequent years have seen new contributions to research and scholarly work that continues to establish student affairs as a strong entity at almost every college and university within the U.S. In addition, strategic connections with international colleagues have also expanded the dialogue to include an assessment of student services at non-U.S. colleges, what may be learned from such a comparison, and innovative ways our institution can work together to prepare our students to be global citizens (Altbach, 2003; Braskamp, 2008; Schulz, Lee, Cantwell, McClellan, \& Woodard, 2007).

A general understanding of the student affairs profession within the U.S. is one that focuses on college student development that occurs both inside and outside of the traditional academic classroom. Quite simply, student affairs services focus on the whole student (Dungy, 2003). Historical accounts of early student affairs staff depict those who enforced campus regulations and student conduct (Thelin, 2011) and are often remembered as staff who served in loco parentis (Rhatigan, 2000). Other origins of student affairs work developed out of counseling and advising (Love, 2003). The field has continued to grow and evolve to present day.

Individuals attracted to the profession are those who seek to be engaged on a college campus (Forney, 1994). Although not an exhaustive list, student affairs divisions and departments may include some of the following student services: "enrollment management, financial aid, housing, counseling, student health, judicial programs, career services, recreational sports, and student activities...transportation, security, child care, and student academic support" 
(Sandeen, 2004, p. 30). Given the variety of jobs within student affairs, it is important that individuals are well-informed of their options, how the different units collaborate, and where professionals may find support once they are working in the field. Weidman et al. (2001) identify the core elements of socialization as knowledge acquisition, investment, and involvement.

\section{Knowledge Acquisition and Investment}

Many elements have contributed to the motivation for student affairs practice, such as the formation of graduate preparation programs to provide formal education to students interested in entering the student affairs profession (Taub \& McEwen, 2006). The pipeline into the student affairs profession is typically quite linear, as most new professionals transition directly from graduate preparation programs in higher education and student affairs. It is important to examine those who self-select a student affairs career path, and there are many contributing factors that lead an undergraduate student to apply and enroll in a higher education and student affairs graduate program. Taub and McEwen (2006) found that a large percentage of students entered the higher education and student affairs profession due to positive influence of senior student affairs officers.

There are currently 359 student affairs graduate programs listed in the NASPA directory (NASPA, 2017c). Most programs rely heavily on providing students with practical opportunities to obtain relevant work experience through internships, practicums, and graduate assistantships. Through such experiences, graduate students typically interact with a variety of campus leaders and administrators as they assume the "roles of both a professional-in-training and a student" (Grube, Cedarholm, Jones, \& Dunn, 2005, p. 152). Additionally, attempts to link theory to practice remains at the forefront of importance to student affairs professionals (Janosik, Cooper, 
Saunders, \& Hirt, 2014; Manning, Kinzie, \& Schuh, 2015; Reason \& Kimball, 2012), thus courses are also devoted to student development theories.

Although actual courses and program requirements may differ between programs, a central theme of graduate students' socialization is critical. Sallee (2011) identified the key players who aid in the facilitation of successful socialization, including faculty members teaching students the norms and values of their chosen field and within their specific departments. Staff members at all levels seek innovative and effective ways to support students. Investment in the graduate student experience is vital to not only to prepare individuals for employment but to set the stage for a productive and fulfilling career, essentially welcoming them into a new community (Golde, 1998). A remaining question for student affairs professionals is how to support ourselves, specifically, our own development.

\section{Involvement}

The creation and subsequent growth of professional associations within the field has increased professional development opportunities for student affairs professionals. Maurer and Sheets (1998) discovered that seven out of ten Americans belong to a professional association (some even more than one) and student affairs professionals are no different. Many belong to one of two large, comprehensive professional organizations, ACPA: College Student Educators International and NASPA: Student Affairs Administrators in Higher Education (Dungy, 2003). Graduate students are socialized into the profession through involvement with these associations (Gardner \& Barnes, 2007). Advanced practitioners continue this engagement with the profession as "a source of renewal, a symbol of resilience and an opportunity to develop intellectually, as well as professionally, and to remember the value of the university" (Schwartz \& Bryan, 1998, 
p.12). Current membership in ACPA and NASPA stands at over 6,000 and over 15,000 respectively, a sign of their perceived importance to practitioners working in student affairs.

\section{Competence and Stages of Career Development}

Providing insight as to why this study seeks to examine current levels of competence in women midlevel student affairs practitioners requires a thorough summary of the history of the evolution of competencies for student affairs practitioners. A variety of definitions and interpretations exist for the terms competence and competencies and it is important to clarify the difference, particularly within the context of higher education. Competence has been defined using terms such as knowledge, abilities, qualities, and values necessary to achieve a goal (Haessig \& La Potin, 2000; Otter, 1995; Wheeler \& Haertel, 1993). Competencies may be described as expertise that is directly transferrable to a specific employment field (Banta, 2001) but are still specific enough to be assessed and easily measured (Voorhees, 2001). For a profession that is as large and complex as student affairs, a recently developed formal competencies framework (ACPA/NASPA, 2015) may be useful in guiding practitioners in competency attainment and progression through the profession. However, no empirical evidence currently exists to support the actual competencies, thus more research is needed on individual practitioners within the field.

Although the release of formal competencies for student affairs is a recent development, the idea of competencies has been discussed by student affairs practitioners for several decades (Burkard, Cole, Ott, \& Stoflet, 2005; Cuyjet, Longwell-Grice, \& Molina, 2009; Hoffman \& Bresciani, 2012; Renn \& Jessup-Anger, 2008). An exploration of the literature finds varied perspectives of key stakeholders regarding preferred proficiencies, skills, and experiences that student affairs professionals should possess. Studies exist from the perspective of new 
professionals (Kuk, Cobb, \& Forrest, 2007) and hiring managers (Kretovics, 2002). One study compared perspectives of senior administrators and faculty who teach in the graduate preparation programs (Dickerson et al., 2011). Interestingly, many of these studies were conducted prior to 2010, the year that professional competency areas were initially created and distributed. More recent studies focus on the application of the competencies by student affairs departments and divisions (Chapman, 2014), measuring competencies (Sriram, 2014), and perceived skill deficiencies among graduate preparation programs (Cooper, Mitchell, Eckerle, \& Martin, 2016). To explore the competency discourse more closely, it is pertinent to consider perspectives from the different career stages typically found in the student affairs profession.

\section{Graduate Student to Early Career}

New professionals comprise $15-20 \%$ of the student affairs workforce and come directly from graduate school, thus requiring a close connection between curriculum and skills needed to be successful in their first position (Cilente et al., 2006). For some graduates of preparation programs, it may be easier to explain competency levels to employers who are looking to hire well-rounded staff as opposed to strictly reporting a list of completed courses and grades earned. A study by Renn and Jessup-Anger (2008) encouraged faculty and graduate students to take a more holistic approach to early career development, considering graduate preparation and the transition to the first job as a seamless learning experience. Advisors serve as the primary agent in this process, as positive relationships between advisors and students facilitate a smooth trajectory (Golde, 2000; Golde \& Dore, 2001). Because competencies emphasize student learning outcomes (Voorhees, 2001), utilizing them also portrays the student as an invested stakeholder in his or her educational experience. 
When attempting to understand exactly what hiring managers are seeking when they interview graduate students for entry level student affairs positions, it makes sense to survey those individuals. Kretovics (2002) found that counseling and helping skills are important competencies for hiring managers to see when they interview graduate students for entry-level positions, and his study reinforced the significance in linking graduate school curriculum with the profession. In their study of Senior Student Affairs Officers (SSAOs), midlevel managers, and faculty, Kuk, Cobb, and Forrest (2007) found these groups desired that new professionals have the capabilities to apply their knowledge to practice. Suggesting that this begins in the graduate preparation programs, Mather, Smith, and Skipper (2010) proposed that "providing more theory to practice opportunities for students can enhance classroom experiences" (p. 14). Like Kretovics (2002), Burkard, Cole, Ott, and Stoflet (2005) found that helping skills are especially important when they examined perceptions of midlevel and senior level student affairs administrators of the positions, responsibilities, competencies, and theories pertinent for new professionals. The same skills were mentioned as preferred when comparing faculty and SSAO expectations for new professional competencies, but they also found that "both faculty and senior student affairs officers perceived large gaps...related to fiscal management, legal standards, and assessment" (Dickerson et al., 2011, p. 475).

\section{Insights from Midlevel and SSAO Professionals}

Following the career progression in student affairs to the mid and SSAO levels, there is less literature. In fact, rather than exploring competencies necessary for career advancement to reach these higher-level positions, most studies involved midlevel professionals and SSAOs strictly for their opinion on the competencies others should have. Thus, the following research 
depicts insights from midlevel and upper-level student affairs professionals regarding competencies needed to be successful in student affairs.

Midlevel. Interested in decreasing attrition of new professionals, Tull (2006) studied synergistic supervision in dyads of supervisors and new professionals, revealing a preference for strong communication and clarification of expectations. Administrators who participated in the Cuyjet, Longwell-Rice, and Molina study (2009) highly rated competencies such as multicultural development issues and working with diverse populations on the job, whereas the same competencies were not as highly rated for inclusion in HESA programs. During a time when the term global citizenship is prevalent in higher education conversations (Lilley, Barker, \& Harris, 2016), leadership in student affairs is urged to provide a supportive environment for the exploration of global competencies (Bresciani, 2008). Finally, Hoffman and Bresciani (2012) examined job postings to determine the competencies most sought-after in student affairs professionals, finding that most competency areas aligned well with those generated by ACPA and NASPA (2010a; 2015).

SSAOs. The highest position in student affairs organizations usually comes with the title of senior student affairs officer, or SSAO. These individuals typically have over ten years of professional experience (Scott, 2000), and oversee all the functional areas of student affairs within an institution, requiring an embodiment of a multitude of roles (Sandeen, 1991). This group may also be referred to as seasoned professionals due to their combination of years in the field and variety of practical experiences. Accordingly, some studies have been dedicated to seeking insight from this group in terms of career advice (Jones \& Komives, 2001; Rickard, 1985). Herdlein (2004) revealed overall satisfaction by senior administrators with the learning outcomes of graduate students, but noted consistent concerns related to courses missing from the 
preparation programs and areas of development for practitioners to be successful. Related to The Professional Competency Areas for Student Affairs Educators (ACPA/NASPA, 2015), Chapman (2014) found that SSAOs rated all ten competencies as "very important or critically important, lending credence to the validity of the competencies" (p. 80).

\section{Professional Competency Areas for Student Affairs Educators}

As the above section explained, conversations about competencies needed to be successful in student affairs are well-established. However, the numerous studies produced an extensive list of the exact competency terms and descriptions. Regarding implementing a competency-based approach to any field, the first step is to define the actual competencies (Ott, Baca, Cisneros, \& Bates, 2015).

Creation. Prior to the 2010, there were no formal competencies for the student affairs field, only guidelines for the closely associated graduate preparation programs. Initially created in 1964, and most recently revised in 2012, the Council for the Advancement of Professional Standards (CAS) offers professional standards for master's level student affairs professional preparation programs. Additionally, in 2010, the Council for the Advancement of Higher Education Professionals (CAHEP) published a set of baseline recommendations for the graduate programs (Freeman, Hagedorn, Goodchild, \& Wright, 2014). Conversations continue around the responsibility of the graduate programs to prepare new professionals, but Tull and Kuk (2012) asserted the need for collaboration among the programs, practitioners, and professional associations. Formal dialogue between the two largest comprehensive professional associations for student affairs, ACPA and NASPA, peaked in 2009 and 2010 through the work of two separate working groups. The first group, named the Task Force on the Future of Student Affairs, 
considered current trends that may impact the field and recommended a renewed focus on the mission and values of student affairs (ACPA/NASPA, 2010b).

The progression towards defining competencies came in the creation of the first competencies task force, called the Joint Task Force on Professional Competencies and Standards (ACPA/NASPA, 2010a). Through in-person and conference call meetings, the group established a set of ten professional competencies to guide the field. In addition to a definition, each competency area is accompanied by a list of skills expected to be demonstrated at the basic, intermediate, and advanced levels. After a period of feedback and revisions, the document was passed by each of the association's governing board and officially endorsed in July 2010 (ACPA/NASPA, 2010a). The "competencies represent a significant step forward for the profession because they are a synthesis of decades of research and provide specific areas of focus for professionals" (Sriram, 2014, p. 350).

In 2014, the second competencies task force was assembled to review the initial document and suggest modifications. A guiding objective of their work was "consideration of applications of the competencies to practice, professional development, and the preparation of new professionals through graduate study" (ACPA/NASPA, 2015, p. 4). Meetings were held in person, as well as online. Formally adopted by both associations in July 2015, the document still consists of ten areas of competence. Although none of the initial competencies from the 2010 document was removed, some rewording and combining of competency areas took place, and one was added (ACPA/NASPA, 2015). A list of the ten competencies and highlights from their descriptions is provided below.

- Personal and Ethical Foundations (PEF): maintaining integrity in one's life and work; standard of ethics; progress towards a higher order of critique and self-awareness; 
- Values, Philosophy, and History (VPH): connecting current practice to the foundations of the student affairs field, including using this understanding to inform research, scholarship, and practice;

- Assessment, Evaluation, and Research (AER): use of various methods and ability to use results to inform practice at small-scale as well as across functional areas;

- Law, Policy, and Governance (LPG): understanding and use of governance structures and policy issues, and their intersection with individual practice, as well as one's organization and beyond;

- Organizational and Human Resources (OHR): management of human capital, financial, and physical resources as well as growth in managing, supervision, conflict resolution, finances, technology, and politics;

- Leadership (LEAD): related to individual leaders and leadership as a process through work with students, colleagues, internal, and external stakeholders, as well as fostering leadership among others;

- Social Justice and Inclusion (SJI): creation of learning environments that foster equity while addressing the need to understand oppression, privilege, and power before understanding the leadership and advocacy that comes with social justice;

- Student Learning and Development (SLD): critical understanding of development theories, their application, and use in application, including the development of learning outcomes;

- Technology (TECH): use of various tools and resources to advance student learning and success, as well as digital literacy and citizenship through innovation and engagement; 
- Advising and Supporting (A/S): direction, feedback, and guidance to individuals and groups and a commitment to holistic wellness in self and others (ACPA \& NASPA, 2015).

A noticeable change in the 2015 version was in the renaming of the levels in which practitioners may progress through individual competencies. The 2010 document provided basic, intermediate, and advanced levels (ACPA/NASPA, 2010a). The 2015 document now provides outcome statements (rather than levels), asserting that student affairs professionals should be able to effectively demonstrate progress in foundational, intermediate, and advanced categories. Important to note is that advancement through the categories will not necessarily align with a practitioner's job title, responsibilities of their position, education level, maturity level, age, nor years in the field (ACPA/NASPA, 2015). This change in verbiage assists in clarifying how the competencies may be attained, and is especially pertinent to this study.

Implementation. Addressing the application of competencies to individual professional development is part of a recent effort to gain buy-in from student affairs professionals. Essentially, now that the document exists, practitioners seek insight for utilization in their own career. The Professional Competency Areas for Student Affairs Educators (ACPA/NASPA, 2015) document includes a section on implications and application, encouraging using the competencies in (a) professional development planning by individual practitioners; (b) recruiting, hiring, and performance reviews; (c) through incorporation by graduate programs; (d) by the professional associations; and (e) to advocate for the profession. Currently, there is no published research on how to apply the Professional Competency Areas for Student Affairs Educators (2015) and this is certainly a needed area for exploration. 
Some research (unspecific to the 2015 document) does exist in terms of using competencies in various capacities. Bers (2001) wrote that faculty should map competencies to courses and learning activities. In 2014, Sriram developed the National Survey of Student Affairs Professionals (NSSAP), an instrument to measure student affairs competencies. In addition, ACPA and NASPA commissioned a joint task force that is currently working on a rubric for

using the competencies (J. Hoffman, personal communication, May 24, 2016). Finally, although not research based, NASPA's Professional Standards Division hosts a resources section on their website to showcase innovative uses of the professional competency areas. Practitioners may submit examples of how the competencies are being incorporated at the individual or program level, as well as within larger departments and divisions.

There is much to be learned from sharing best practices among colleagues and institutions in terms of best practices in applying competencies. Hoffman and Bresciani (2012) share that, over time, expertise and skill in a competency area continues to build. Thus, time in the profession combined with a variety of professional experiences could contribute to competency attainment, which could then contribute to professional mobility. Still absent, though, is how seasoned professionals go about developing competency. This study represents one attempt to fill that gap.

\section{Women Midlevel Student Affairs Practitioners}

Relevant to the discourse on competency development is a focus on practitioners themselves, and within student affairs, several sub-groups of professionals exist (Sandeen \& Barr, 2014). Practitioners may associate with groups based on (a) the type of work they do or their focus area/functional unit they represent; (b) working at similar institution types or sizes; 
(c) working in the same geographical region; (d) based on personal identity or professional level (Gerda, 2006; Renn \& Hodges, 2007).

Interested in two of these sub-groups, this study combines the identities of gender and career stage as the focus population. The result is an examination of women midlevel student affairs practitioners. Although numerous studies have focused on women in the student affairs field (Blackhurst, Brandt, \& Kalinowski, 1998; Jones \& Komives, 2001; McNair, Miguel, Sobers-Young, Bechtel, \& Jacobson, 2013; Sandeen, 1991; Yakaboski \& Donahoo, 2011), midlevel practitioners are currently underrepresented in student affairs research (Young, 2007).

\section{Women}

Some advances have been noted about women reaching administrative positions within higher education, yet challenges remain in terms of women seeking advanced degrees, career advancement decisions, and balancing the needs of family (Barr, 2004). Numerous conversations on these topics occur informally on campus, and even formally through roundtables and presentations at professional conferences. A highly-regarded leadership training for women in higher education, which includes student affairs leaders, the Higher Education Resource Service (HERS) hosts three professional development opportunities each year. In addition, both leading student affairs professional associations have dedicated opportunities for women to interact, mentor, and learn from one another. Examples include the ACPA Coalition for Women's Identities (CWI), the NASPA Coalition for Women, and the NASPA Women in Student Affairs (WISA) Knowledge Community. These groups coordinate professional development programs such as symposiums, institutes, lunch and learn series, candid conversations, awards programs, and writing workshops. There is also a journal dedicated to women in higher education, NASPA Journal About Women in Higher Education (NJAWHE), which "focuses on issues affecting all 
women in higher education: students; student affairs staff; faculty, and other administrative groups" (NASPA, 2017b). Still, it is difficult to find scholarship solely dedicated to women practitioners in student affairs.

\section{Midlevel}

Typically reporting to the top student affairs administrator, and responsible for one or more functions of their student affairs division, is the midlevel professional (Fey \& Carpenter, 1996). Considered by some as middle management (Mills, 2007), this tier of professionals occupies an important space in between entry-level staff and chief administrators. Sometimes referred to as the number two person in charge indicates these individuals have been entrusted with responsibility, but they are not yet the key decision maker (Ackerman, 2007). Essentially, those in midlevel positions hover in the space between, and at least one study reported low levels of both organizational commitment and life satisfaction when holding the assistant or associate title (Blackhurst et al., 1998).

There is some dedicated support for midlevel leaders. ACPA provides resources for a group they call the Midlevel Community of Practice (ACPA, 2017). Several regions within NASPA coordinate a special conference for midlevel practitioners, the Mid-Managers Institute (MMI) or the Midlevel Institute (MLI). At their 2016 regional conference, Region IV-West described the initiative as 'intended for professionals with a minimum of five years' full-time experience and supervisory, budget and/or programming responsibilities for a department" (NASPA, 2017a, para. 1).

While Young (1990) focused primarily on the composition of mid-career professionals, few scholars have concerned themselves with the actual practitioner experiences at this stage of career development in student affairs. In 2004, Barr clearly stated that attention has been given to 
senior student affairs officers and new professionals, yet "little has been written" about the midcareer in student affairs. Within the last decade, however, NASPA sponsored a book project that revisited Young's work on midlevel managers in student affairs. Ackerman (2007) asked Young to present the five assumptions that inspired his initial research on this populations. Per Young, student affairs middle managers: (a) are too important to be ignored; (b) have no identity in the middle; (c) get no respect; (d) are dissatisfied with their jobs; and feel stuck on the career ladder. These assumptions provide justification and motivation to further examine this group.

\section{Women Midlevel}

Given this study's interest in professionals who identify as both a woman and a midlevel student affairs practitioner, a thorough search of the literature to find information applicable to the group was necessary. As explained in the above section, scholarship on women in student affairs is plentiful, and there is limited scholarship on midlevel professionals. When searching for publications that examine women and midlevel as one entity, only a few studies may be found. For example, from their qualitative study on this group, Belch and Strange (1995) found that women at the midlevel report struggle with constant challenge to devote necessary time to work and non-work commitments. However, the only stand-alone publication that specifically includes stories about midlevel women navigating a student affairs career, and therefore of great relevance to this study, is Roads Taken (Renn \& Hughes, 2004).

Roads taken. Through a collection of stories from over 20 women in the mid-career phase of student affairs, the book covers topics including considerations of advanced degrees, dual-career couples, motherhood and student affairs, and reaching professional milestones, as well as alternate routes within the student affairs career. Their chapters help us understand this group better through the authors' approach of eliciting actual lived experiences from women, 
then sharing the experiences in their own words. Several stories from Roads Taken are especially pertinent to this study's focus on what constitutes a successful career for women midlevel practitioners, and the role gender has played.

Education. Once reaching midlevel, professionals may consider taking classes towards a certificate or advanced degree as way to obtain new skills or earn credentials that could assist in career mobility. Howard-Hamilton (2004) wrote of her decision to leave her professional position to pursue her doctorate as a full-time student as "one of the toughest decisions a person makes is whether or not a terminal degree is needed to make it through the academic ranks or organizational pipeline" (p. 4). Adding to the perspective that such a decision is highly personal in nature, Jones (2004) discussed the importance of timing when enrolling in a program, as well as the community needed to support one through the process. Wong (2004) honestly shared the numerous obstacles that may impact educational pursuits, such as "career, family, and physical and emotional well-being" (p. 37). This last statement is but a short list of obstacles that may often hinder student affairs professionals from enjoying stability in their overall lives. These stories about pursuing educational endeavors at the midlevel are indicative of decisions that are far from simple; decisions that take multiple factors into consideration.

Personal and professional life balance. The various job responsibilities and often nontraditional work schedule (e.g., evening, weekend, intense summers) of student affairs professionals have the potential to impact their personal lives (Guthrie, Woods, Cusker, \& Gregory, 2005). Further complicating attempts to achieve some sense of balance between their personal and professional lives, many practitioners have partners, children, parents, and others who depend on them. Three chapters of Roads Taken are devoted to stories of navigating dual- 
career circumstances, and the concessions that are often made by one partner as a sacrifice to the other's career.

Marshall (2004) addressed the challenges of working mothers sharing, "in spite of increased numbers of women in administration, little attention has been paid to how women manage the complexity of a career in higher education and a family" (p. 91). Reflecting on the dichotomy of managing multiple roles and demands, Joyce-Brady (2004) titled a section of chapter, quite simply, "they said a woman could do it all" (p. 113). Of course, precisely defining what "it all" encompasses is purely individual in nature. These stories of women at midlevel needing and seeking balance emphasize the numerous barriers that must be navigated in order to somewhat separate personal time from professional responsibilities.

Career development. The end career goal in student affairs varies among women. While some may strive to reach a position as a top administrator, others may be comfortable at the midlevel stage. Some may take an alternative route into a faculty position or other academic role, and yet other practitioners may eventually transition out of student affairs and higher education altogether. Many of such decisions are impacted by the culture of one's workplace. For instance, Horton (2004) referred to a time she was offered a unique job share position with a colleague, which is uncommon for this field; she also wrote of another position that allowed her to use her "lived skills" (p. 155) as she simultaneously expanded her skillset. As Olyha (2004) navigated her own career development, she benefitted from the positive impact of a women's mentoring group. Ortiz (2004) expanded on the role of colleagues, supervisors, and mentors in her discussion of the importance of socialization into the career expectations of the field. These stories highlight the unique ways some women have taken strategic steps to advance their own careers. 
Collectively, the individual stories told in Roads Taken tell us that no career looks the same for women at the midpoint. Decisions are made with a multitude of factors in mind, there is a strong need for balance between their personal and professional time and responsibilities, and there are times when individuals must take charge of their own career decisions in order to have new opportunities or to advance in the profession. The overarching theme is that women midlevels professionals' careers are complex, and furthermore, their lived experiences deepen the understanding of these complexities.

\section{Importance of Additional Research}

Essentially, there is no simple way to understand and explain the midlevel career point of women student affairs practitioners. Highlighting the unique complexities that accompany this status, Hughes (2004) asked:

How is it that just when we manage to establish ourselves in our chosen profession, figure out how to make the work intersect with the rest of our lives, and finally have developed professional depth, we are confronted with mid-life and all of its attendant angst and reflection? (p. 136)

Combining aspects of gender and professional level as a research topic may be more challenging than examining each group separately, yet the potential implications that may come from such research supports such endeavors. Hirsch and Berman-Hills (2004) wrote of the many hurdles that exist in women's career development, but that women are indeed successful. Similar sentiments may also be applied to the task of researching this population, provided the project is approached with complementary methodology, clear purpose and significance, and an appropriate framework. The framework for this study ambitiously takes on the intricacies of career decisions made by women. The framework includes parameters that are not only 
applicable to what we have learned from existing literature, but bring new perspectives on how women midlevel student affairs practitioners develop the competencies for a successful career.

\section{Overview of Theoretical Framework: The Kaleidoscope Career Model}

As previously stated, my review of the literature identified several gaps regarding how individuals working in student affairs build the competence needed for a career they (and others) deem successful. Additionally, formal competencies created for the profession do not account for the unique and personal experiences of individual practitioners, nor do they consider gender. Thus, the purpose of this narrative inquiry is to create a new thread within the competency literature specifically pertaining to the career development of women, midlevel student affairs practitioners. The Kaleidoscope Career Model (Mainiero \& Sullivan, 2005; 2006) is used as a theoretical framework for this study. Specifically, the KCM aspects of authenticity, balance, and challenge are used to understand career experiences and career paths as told by the participants.

The Kaleidoscope Career Model (KCM) was created by two women faculty members from the business management discipline (Mainiero \& Sullivan, 2005; 2006). The contribution of the KCM is that it "provides context to the study of careers and puts gender in the foreground" (Mainiero \& Sullivan, 2005, p. 113). Identifying a need to learn more about women's decisions to leave their careers served as the impetus for Mainiero and Sullivan's (2005) collaborative work. Among many revelations, they found that women view each career-related action as having lasting effects on those around them, and as such, decisions are carefully considered with the focus more on others than themselves. To explain this concept of connectedness, they equate a woman's career decisions to a kaleidoscope:

Like a kaleidoscope that produces changing patterns when the tube is rotated and its glass chips fall into new arrangements, women shift the pattern of their careers 
by rotating different aspects in their lives to arrange their roles and relationships in new ways. (p. 111)

As this description illustrates, as one part of a kaleidoscope rotates, the movement causes simultaneous change to everything that is connected.

The KCM is grounded in research that found dramatic differences in the careers of men and women, with those of women more relational in nature. Thus, KCM takes the perspective that women's career decisions are typically connected to other issues and people, and more often made from a lens of relationalism. This perspective of women's decision-making differing from that of men connects to the work of influential feminist authors who reconfigured traditional theories by adding the perspective of gender.

For example, Gilligan's renowned publication, In A Different Voice (1982) emphasized differences in how men and women think and act, with the style of women considering issues more with care and responsibility (as opposed to rights and rules). Building upon Gilligan's work, Belenky, Clinchy, Goldberger, \& Tarule (1986) identified five stages through which women look at reality. Though all five stages relate to the $\mathrm{KCM}$, it is the fifth stage (i.e., constructed knowledge) presented by Belenky et al. (1986) that most closely relates to the kaleidoscope metaphor, described as making decisions while concurrently a "daughter, mother, lover, nurturer, thinker, artist, advocate" (p. 137). Finally, Love and Guthrie (1999) discussed the implications of relational research for student affairs professionals, providing several recommendations specific to women practitioners. It is noteworthy, that this body of scholarship is not without its critiques, as many scholars argue that viewing women as relationship oriented essentializes them, suggesting that these perspectives are true for all women (Broughton, 1992; Davis, 1992; Kerber, 1986). 
In terms of men and women having different vantage points for career perspectives Powell and Mainiero (1992) assert that in general, women's lives do not fit into the typical stages of a traditional career. Related to is point, a 2003 study by O'Neil, Bilimoria, and Saatcioglu classified women into different categories based on how they make career decisions, with most women having careers described as without boundaries. Thus, upon the creation of the KCM, the framework has been used to study the needs of women professionals (Sullivan \& Mainiero, 2008). Considering the metaphor of the kaleidoscope for working women, there is much give and take when it comes to making career decisions. Women tend to modify their careers by rotating different aspects of their personal lives (Sullivan, Forret, Mainiero, \& Terjersen, 2007). Exactly what this looks like certainly varies between individual women, but the KCM identifies common reasons that led to a kaleidoscope career. Created on their own terms, kaleidoscope careers are fluid and in motion, and alter to adjust to life changes (Sullivan et al., 2007).

From my professional experiences, I am keenly aware of the complexities of a career in student affairs, particularly one that evolves over time in terms of responsibilities. I am also attentive to the demands that may accompany a woman in the mid-career stage of life. As such, KCM's three key parameters hold great potential in furthering our understanding of the career development of women who find themselves at a crossroads based on gender, career stage, and the personal and professional demands that accompany this identity.

\section{Parameters of the KCM}

Participants in the studies conducted by Mainiero and Sullivan (2005) "not only considered the impact of their decisions on others, but also whether their choices were true to who they are, their vision for work/non-work balance, and their need for challenging work" (p. 113). Thus, the 
model identifies three crucial career issues that women face in career development: authenticity, balance, and challenge (Mainiero \& Sullivan, 2005, 2006; Sullivan et al., 2007):

- Authenticity relates to the need to be true to oneself, genuine, and to align behaviors and attitudes with personal values, perhaps leading them to pursue their own path;

- Balance is the need to satisfactorily manage both work and life/family, and to create a healthy equilibrium among work, relationships, caregiving roles, and other non-work aspects of life, and may result in using strategies such as adjusting their work time through part-time employment, opting out from the workforce temporarily, taking turns to opt out with their spouses, and arranging workloads in accordance with family situations;

- Challenge is the need to improve oneself, striving for stimulating work (and stimulating assignments on the job), career advancement, and self-worth, and may include individuals who are willing to go beyond at work.

While the three parameters may coexist, there is typically one parameter that is used predominately over the others at any given time in a person's career.

Continuing with the metaphor of a kaleidoscope to better explain this dynamic, Sullivan et al. (2007) used an artistic metaphor:

...the colors of an individual's kaleidoscope are reflected in these three parameters, shaping decisions as one aspect of the kaleidoscope, or color, takes on greater intensity as a decision parameter at different points of the life span. Over the course of the life span, as a person searches for the best fit that matches the character and context of his/her (sic.) life, the colors of the kaleidoscope shift in response, with one color (parameter) moving to the foreground and intensifying in color as that parameter takes priority at that time in 
his/her (sic.) life. The other two colors (parameters) lessen in intensity and recede to the background, but are still present and active as all aspects are necessary to create the current pattern of an individual's life/career. (p. 6)

Applying the unique metaphor of a kaleidoscope to study women's careers is both unique and practical. The KCM holds great promise for examining the complexities of a women's career in student affairs, mainly because of its attendance to the multiple aspects that influence career decisions. Important first is to understand how the KCM has been incorporated into previous studies from various disciplines outside of education, and a few within.

\section{Applying the KCM to Research}

Exploring the literature for the KCM results in hundreds of articles that referenced the model, mainly from the fields of business, career development, human resources, counseling, and organizational psychology. Once the $\mathrm{KCM}$ was created, its authors continued to work together to publish several journal articles explaining the model, and others that use the model to address other issues related to careers. Two examples of Sullivan and Mainiero's collaborations (within the same year) used the KCM to explore issues such as: examining women's stress (2007), benchmarking ideas for fostering family-friendly workplaces (2007), and the design of human resource development programs to retain woman (2008). They have also partnered with other colleagues on projects using the KCM (Sullivan et al., 2007; Forret, Sullivan, \& Mainiero, 2010, \& Sullivan, Forret, Carraher, \& Mainiero, 2009).

Five published dissertations have used the KCM, also from the business and career related disciplines listed above. In 2010, Gagnon used the KCM and several other career models to assess resiliency among mid-career managers who had been unemployed long-term; the study suggested that adopting a combination of alternative career models could be helpful as 
individuals develop strategies to meet their personal career needs. Aprianingsih (2012) explored work family conflict across career stages, finding that authenticity and balance were positively related to work family conflict, indicating that gender and career stage pertain to perceptions of work family conflict. Also in 2012, Simmons applied the KCM to a study on networking, finding a link between the parameters of the KCM and how individuals target their networking behaviors to help achieve their career goals. Two international studies employed the KCM. First, Yourston (2016) investigated generational differences in British managers, finding the KCM allows one to view differences towards career success from a generational perspective, but that factors such as type of profession, gender and stage of life are related. Braches (2015) examined gender in career transitions from corporate management to entrepreneurship, finding that women in Germany experienced gendered elements within the traditional careers offered to them, such as women being viewed as caretakers as one example, and that such elements pushed them to pursue a more boundaryless career path in entrepreneurship .

Limited use of the KCM is noted with the education field, though some instances are noted. Carraher, Crocitto, and Sullivan (2014) studied decisions surrounding faculty sabbaticals, and suggested the KCM as a helpful model for individuals to determine the feasibility and desirability of taking a sabbatical. Two published dissertation studies from education have also used the KCM. Ervin's (2015) exploration of specific career needs of intercollegiate head coaches, finding the challenge career need best described coaches, and that coaches' need for authenticity was more important in early career. Tajlili's (2015) used the KCM to create an instrument (the Future Work-Life Balance Attitudes Scale) to assess millennial college women's attitudes on work-life balance, the first work-life balance instrument of its kind geared toward the college women population. 
Although these studies used the KCM in various ways and to different degrees, some common themes are noted. The parameters of the KCM seem helpful when investigating complex career decisions, especially in situations where one choice impacts other aspects of a person's life. The KCM has also been useful in exploring a specific profession, and within the profession, differences in perceptions and needs between genders. The model has proven beneficial in creating assessments, and use of the KCM both inside and outside of its home discipline has advanced the model by viewing it from new perspectives and applying it to new populations. Building upon this last concept is this study's application of the KCM to the field of student affairs.

\section{KCM and Student Affairs}

A clear link between the KCM and student affairs relates to women who depart from their intended career path. Several studies within student affairs have attempted to identify factors that contribute to low job satisfaction, attrition, and even a departure from the field. In a national study of midlevel student affairs professionals, Rosser and Javinar (2003) demonstrated the link between job satisfaction and morale with one's decision to remain in the career, or not. As mentioned earlier in the literature review, the traditional student affairs career path was designed to be quite linear. Perhaps this linearity has outlived its usefulness, particularly as both campus cultures and our society has evolved in multiple capacities since the field was established. In fact, the mid-career stage has been equated to that of a bottleneck (Belch \& Strange, 1995), reflecting the complexities of career decisions made at this juncture.

My study is the first I was able to find within student affairs to use the $\mathrm{KCM}$ as a guiding framework, thus providing a unique opportunity to further discuss the commonalities faced by women professionals at the mid-career stage, no matter their discipline or industry. Furthermore, 
the decision to use a framework outside of education aligns with the methodology selected for this study, as narrative inquiry is inherently interdisciplinary (Riessman, 1993). This study is an example of the benefit in blending $\mathrm{KCM}$ as a guiding framework and narrative inquiry as the method of data collection, emphasizing the potential for new understandings when an interdisciplinary approach is taken.

\section{Summary}

The review of the literature served as an introduction to the student affairs profession and both the historical and current discourse within the field on competencies, as well as women midlevel practitioners. Gaps exists in relation to empirical research on competencies and ways they can be applied to professional development. Further gaps are noted among the research of women at midcareer. Finally, I introduced the KCM as a new way to frame student affairs studies. The next chapter provides a comprehensive overview of how narrative inquiry best supports the purpose of this study, and reaches the participants in a personal way that not only helps answer the research questions, but leads to significant findings with implications for research, practice, and theory. 


\section{Chapter 3: Methodology and Research Design}

The purpose of this qualitative study is twofold: 1) to use narrative inquiry to explore how women, midlevel student affairs practitioners have developed the competencies needed for career success, paying attention to the role of gender in their career development; 2) to use my findings to contribute to the research and discourse on career development in the field of student affairs by focusing on midlevel professionals. Merriam (2009) described four key characteristics of qualitative research: "the focus is on process, understanding, and meaning; the researcher is the primary instrument of data collection and analysis; the process is inductive; and the product is richly descriptive" (p. 14). I designed a study that centered the participants' voices, a study where I played an integral role in the meaning making, and a study that revealed a meta-narrative among this group of women that is both compelling and insightful. All aspects of the research methodology and design used in this study are reported in this chapter.

Given that the principal intent of this project was to gain a deep understanding of a complex issue, a qualitative research design allowed me to best answer my research questions. Qualitative research is reflected by a metaphor of "intricate fabric composed of minute threads, many colors, different textures, and various blends of material" (Creswell, 2013, p. 42). Using this metaphor of the fabric is a helpful way to consider the complexities of this study, including the large size and diversity of the student affairs field as well as specific aspects of practitioners' identities such as their gender, personal attributes, approaches to skill development, and view of

success. Researchers use qualitative inquiry to understand individual meaning making or to gain a more sophisticated understanding of others' experiences and ways of thinking (Guba \& Lincoln, 1994; Lincoln \& Guba, 2000), thus providing the best structure for this study. 
Because I am interested in personal stories of student affairs practitioners' career experiences, as told by women, midlevel practitioners, I searched for a methodology that prioritized the participants' voices. Narrative inquiry was the tool used to help each participant tell her story. Narrative can be either verbal or written text that provides a chronological account of a series of events (Czarniawska, 2004). By identifying individuals who graciously allowed me a glimpse into their professional stories through in-depth interviews, I understood more about the complexities of their own career development, and how their stories contribute to how the student affairs field understands this population.

Considering the components typically included in narrative studies, this study is a collection of individuals lived experiences and utilizes different forms of data (Creswell, 2013). Six participants shared their professional stories through various data sources: (a) demographic information collected through a pre-interview questionnaire; (b) participant resumes; (c) interviews; and (d) sketches drawn by participants. Riessman (1993) emphasizes the actual story is the object of narrative analysis. Through the process of collecting stories, researchers are often able to identify turning points (Denzin, 1989), also included on Daiute's (2014) list of plot elements of a narrative. Within each participant's story of her professional journey were many turning points.

The primary method used to examine the data was thematic analysis; I reviewed the data multiple times to build an interpretive understanding of key themes (Geertz, 1973). Upon completion of the reviews, codes were created for each of the six participants. Noting commonalities, codes were then blended to create themes. This process is signified by when the data itself drives the construction of categories (Patton, 1980). The resulting seven major themes 
and corresponding subthemes were then interpreted through this study's theoretical framework, the Kaleidoscope Career Model (KCM) to guide discussion of the findings.

Regarding the intentional choice to study women midlevel professionals, the literature review supported the significance of this population. The organizational structures often found within institutions of higher education have traditionally been dominated by men, with diversification of this important role slower than preferred (June, 2007). Midlevel student affairs women professionals have received little attention, with only two significant works within the last 15 years (Ackerman, 2007; Renn \& Hughes, 2004). The methodological choice of narrative inquiry to understand the experiences of this population was intentional, grounded in its connection to the concept of power and individual agency. According to Andrews, Squire, and Tamboukou (2008) there exists "socially effective agency of personal stories" (p. 7). Individuals tell their own story in only a way they can; the experience is personal. Bruner (1990) privileged the details of the lived experience, particularly as the researcher attempts to make meaning of the information shared. Moreover, considering the aspect of gender, feminist scholars often use narrative to investigate voice (Gilligan, 1982) and as stated, the participants in this study are women, thus the use of narrative is further justified. Collectively, the women in this study have worked for several years in environments steeped with institutional traditions, and while each story encompasses unique variations, each included examples of opportunities and challenges. Related to these experiences about gender representation within higher education and student affairs, the concepts of performance in social roles (Butler, 1993; Goffman, 1959) emerged through the narratives collected for this study. This revelation underscores the emancipatory potential of individuals sharing their stories, and adds an exciting aspect for the use of this study's findings, discussed more in Chapter 6. 


\section{Researcher Positionality}

Researchers position themselves in qualitative studies (Creswell, 2013), and I possess a unique combination of identities and experiences related to this study. Identifying as a woman, my career includes two distinct experiences in higher education. As a former student affairs practitioner and currently a doctoral student of the field, my personal and professional experiences contribute to several aspects of this study. I also bring an epistemology of constructivism that fits with qualitative research and narrative inquiry.

Epistemology attempts to clarify the boundaries of human knowledge, "answering the question of whether social phenomena are real or just a construct of the observer" (Wodak \& Meyer, 2016). More simply put, it is the lens through which we view the world. The constructivist view points to the unique experience of all of us (Crotty, 2013), underscoring the importance of my positionality in this study. Building upon this thought, constructivism in qualitative research suggests that co-constructed realities are developed through the research process. Essentially, that reality is created both by the researcher (myself) and the participants. Examining the role that I play as the researcher in this study is an important aspect of the foundation of the entire project.

As a scholar, I am drawn to the concept of me-search-bringing the "me" into the research, which is just one component of scholarly personal narrative or SPN (Nash \& Bradley, 2012). Pertinent to the student affairs field, this approach emphasizes the value of an individual researching topics that are significantly important to their interests, but also to who they are as a person. To say I am immersed in the student affairs field would be an understatement. At the time of this study, my investment as a higher education and student affairs professional spans 15 years. I spent 12 years as a student affairs practitioner working in a variety of roles at three large 
public institutions in the Southeast and Midwest. But significant to my story are four other individuals.

I met my husband while we were graduate students earning our master's degree in student affairs administration, subsequently navigating the field as a dual-career couple. I am a mother to three young daughters who benefit from growing up in a college town, even attending preschool on campus and on occasion, accompanying us as we travel to professional conferences. I consistently weigh the different aspects of my identity as a practitioner-scholar and as a mother and partner. I reached the midlevel phase of my career development, reporting directly to the chief student affairs officer, in my most recent position. At this 12 -year professional mark, I made a strategic decision to step away from the field to pursue my doctoral degree as a full-time student. I have spent the last 3 years completing coursework in educational leadership and policy analysis, with an emphasis on higher education administration. Throughout my practitioner and academic years, I have remained involved with professional development, presenting regularly on student affairs topics.

Considering the degree to which my positionality influences my study is significant. More than simply choosing a dissertation topic of interest, this project is a calling as I attempt to weave my personal and professional experiences into my research in hopes to making a significant impact in our field. My background serves as the basis of my inquiry and what eventually led to this study. My history in the field has allowed me to build an extensive network that spans institutions across the country, providing access to information and people. Although I am connected to the purpose of the study, I was cognizant not to make early assumptions about the outcome of the study. However, the fact that I share similarities with my research participants in terms of gender, career stage, professional involvement, and family life (for some) certainly 
came into play during the interview process and coding. Articulating my positionality at the start of the study allowed me to be more intentionally reflexive throughout the process so that I was consistently aware of how my positionality influenced the research process. Subsequent sections of this chapter address my approach to trustworthiness, my process for recruiting participants and ensuring confidentiality, and activities that allowed me to bracket my own experiences as I moved through data analysis.

My positionality is also important for my readers. As Wolcott (2010) stated when writing on reflexivity, "our readers have a right to know about us...they want to know what prompts our interest in the topics we investigate... and what we personally stand to gain from our study" (p. 36). My career goals include interests in teaching and administration, thus I am interested in implications for future research as well as implications for practice yielded from this study. Explicitly, I hope this study will provide much needed examination of how the student affairs field understands career development, particularly for women at the midpoint of their careers, as well as the role of gender in career development as told through the lived experiences of practitioners. By sharing findings from this study through conference presentations and scholarly publications, I will articulate the need for additional research on what is needed for a successful career in student affairs. I do not expect this lone study to provide everything the field needs to know on this topic; thus, the study will hopefully serve as a building block for a larger research agenda as I move through my own career development.

\section{Participants and Sampling Procedure}

There are several unique characteristics of narrative research that distinguish it from other qualitative methods, one is that it does not provide "automatic starting or finishing points" (Squire, Andrews, \& Tamboukou, 2008, p.1). Thus, in terms of sample size, it was difficult to 
determine an appropriate guideline. The sample size of six may be viewed by some readers as larger than a typical narrative study, as some examples have as few as one or two narratives (Creswell, 2013). My research into the use of narrative analysis in educational research, however, revealed some larger samples, particularly useful when the researcher is interested in the collective story. For example, Craig (2009) presented a longitudinal study where she consistently interviewed teachers and administrators as a "constellation of stories" (p. 604). This point directly relates to my interest in the meta-narrative as told by the collective voice of my sample. With no clear sample size dictated, I originally designed the study with a goal of collecting narratives from ten participants. I believed this to be a reasonable goal for two reasons: (1) multiple narratives are needed to build a meta-narrative; and (2) the time required to interview and code (I deemed more than ten to be too many).

Several factors contributed to the reduction of my sample from ten to six women. My original list of potential participants would have required me to travel to multiple states across the Midwest and Upper Midwest during the winter season, increasing the likelihood of delays and rescheduling. I also considered conducting interviews in-person at the NASPA annual professional conference attended by thousands of student affairs professionals, given the high likelihood of securing in-person interviews during the conference based on being in the same location for multiple days. However, as the March meeting of this conference approached, I was still in the process of conducting second interviews with my original six participants and did not want to break stride. I was excited with the information I was gathering at the time and believed I was reaching data saturation. Adding more participants in March may have slowed my current progress and would have resulted in a delay of the study's completion by several months. Further, because of data saturation, additional participants would likely only reinforce the 
already emergent themes, making it unneeded methodologically (Cite a source regarding data saturation).

Given the scope and timing of my study, six participants allowed for varied perspectives while also obtaining enough information to answer the research questions that guide this study. My participants share many similarities yet their professional stories take divergent twists and turns, often unexpectedly. The results explained in Chapter 5 reflect common threads found amongst the six stories as well as unique points shared by just two or three. These six stories not only answered my research questions but provided the impetus for ideas for next steps included in Chapter 6.

Just as important as how stories are told through narrative research (Riessman, 2008) is exactly whose voice is being represented. Given the study's primary focus on the specific population of women midlevel student affairs practitioners, I spent significant time reflecting on whom to sample, a process congruent with a narrative study (Creswell, 2013). It was important that I identified participants who fit my distinct criteria, and who I believed would be interested in the study. Alternatively, being too selective would have limited my scope when the long-term goals of the study are to provide insight to the larger field of student affairs. Thus, the participants needed to possess specific criteria, yet have broad and varied experiences. My previous professional experiences in student affairs served to be beneficial during this process. Based on my work history at multiple institutions, extensive involvement with professional associations and conferences, and consistent peer networking, I have acquired hundreds of student affairs connections at institutions ranging in size, demographics, and geographic location and felt confident in their willingness to participate in the study. 
In December 2016, I received IRB approval to recruit participants for my study. I utilized a purposeful sampling (Patton, 2002) to identify the first five participants who fit my distinct criteria. Access and rapport with participants is an essential component of qualitative research, and in narrative inquiry, it is pertinent that participants understand the motivations of the researcher (Creswell, 2013). Participants were identified based on my own awareness of their careers and relative geographic proximity; each participant carried a positive reputation in the field and is anecdotally regarded as successful by peers. When searching for a sixth participant, I asked my current participants for recommendations, and one offered her assistance. This process of network sampling (Schensul, LeCompte, Trotter, Cromley, \& Singer, 1999) allowed me to trust her insight for the suggestion and served as a rapport-builder when I reached out to the individual who I had never met, but ended up being my sixth and final research participant. Using the combination of purposeful and network sampling provided what I needed to recruit and sustain ideal participants for my study.

Because I knew five of the six participants personally and professionally, I felt comfortable with my initial assumption that their gender identification would be as women and based on their years of experience, their current professional level would be midlevel. By designing a brief pre-interview questionnaire to collect demographic data, I obtained additional information about the participants' identities, including race, ethnicity, age, relationship status, and education level. These data are represented later in this chapter as part of my formal introduction of the participants. The next section provides an overview of where the participants are employed. 


\section{Research Context}

My priority for the study was based more on the people rather than institutions, but considering geographic area was beneficial in terms of establishing the process of data collection that was both reasonable and efficient. Given my own location and ability to travel, selecting institutions within driving distance was preferred. Thus, the data collection for this study took place on two college campuses located in different states in the Midwest region of the United States. For confidentiality purposes, the institutions will be referred throughout the study by the following pseudonyms: Flagship University and Midwestern University.

Both institutions are similar in size regarding enrollment as well as student affairs divisions and departments. Both are currently recognized as members of the Association of American Universities (AAU), a symbol of inclusion in a selective group of institutions with positive reputations in research and graduate education (AAU, 2017). Using the Carnegie Classification of Institutions of Higher Education (n.d.) provides a more detailed description of each institution: both are described as doctoral universities with the highest research activity. Flagship University is a 4-year, large, and primarily nonresidential institution with a high undergraduate population. Midwestern University is a 4-year, large, and primarily residential institution with a majority undergraduate population. Four participants are currently employed at Flagship University and two are currently employed at Midwestern University. My rationale for choosing different institutions was my intention to diversify the experiences of the various participants. While I could have chosen a case study approach that included women working at the same institution, instead, and consistent with the notion of a kaleidoscope, this study included narratives from women who have taken varied professional paths and currently work within different campus cultures and organizational structures. 


\section{Data Collection}

Data were gathered through a pre-interview questionnaire, participant resumes, a hard copy sketch drawn by the participant prior to the first interview, and by reviewing transcripts and audio files from interviews. First and second interviews conducted with participants were audiorecorded, and I kept a journal of field notes throughout the data collection process. The following section provides details regarding communication with potential participants, informed consent, interview scheduling, and all aspects of data collection.

Potential participants first received an e-mail of introduction explaining the scope of the project and requesting approximately 60 minutes of their time for an in-person interview (Appendix A). Once a participant indicated interest in participating in the study, I e-mailed the informed consent form (Appendix G). If the potential participant indicated no concerns after reading the informed consent form, I used e-mail and phone to schedule the first interview. Once

the interview was secured, I sent a confirmation e-mail (Appendix B) that included the following information: 1) A link to the pre-interview questionnaire, 2) A request that the participant e-mail me a copy of their current resume, and 3) A copy of the Nyquist et al. (1999) article to provide context to the participant sketch activity I adapted for this study. Included at the bottom of the confirmation e-mail, I encouraged participants to review the information and to contact me should they have questions before our first interview.

\section{Pre-interview Questionnaire}

The purpose of the pre-interview questionnaire was to collect demographic information that assisted me in understanding more about each individual participant. Questions were intentionally designed to capture information salient to the purpose of the study. Using an electronic survey software, Qualtrics, assisted with both the distribution of the survey and the 
collection of responses, and enabled me to apply skip logic to specific questions. Skip logic allows the survey designer to send respondents to a future point in the survey based on how they answer a question (Qualtrics, 2017). For example, if a participant indicated a relationship status of married, they were presented a subsequent question asking them to indicate the career of their spouse; if the participant indicated a relationship status of single, the sub question did not appear. Information collected through the pre-interview questionnaire supported my assumption that the participants indeed met the criteria I set for participants in this study: women at the midlevel point of their career. The information also helped me better understand both the professional and personal identities of my participants prior to sitting down for the in-person interview. A summary of information collected from the pre-interview questionnaire is included late in this chapter.

\section{Resume}

The purpose of collecting the current resumes from each participant was to better understand the educational and professional background that led to the individual reaching this point in her career. All participants e-mailed me a resume, and most were updated to the point that it included their current professional position. I compared experiences of all six participants to identify common aspects across my sample but also noted specific differences. Information from the resumes assisted in the creation of the interview protocol (Appendix C); based upon the information, I was quickly able to inquire about specific professional experiences during the interview. A summary of information collected from participant resumes is included later in this chapter. 


\section{First Interview}

An important aspect of this study was to interview participants in-person and at their home institution. Collecting data in a natural setting is common in qualitative research, as it allows the researcher to talk directly to participants within their context (Creswell, 2013). As noted earlier in this chapter, alternate options for interviewing participants for this project were carefully considered. While phone interviews would be an efficient and cost-effective option, I believed it would present challenges in building rapport with participants. After much consideration, I determined the preferred interview setting is where the participants are most comfortable. Creswell (2013) stated that qualitative study requires researchers to "commit to extensive time in the field" (p. 49) and I made a personal commitment to fulfill this requirement by adjusting my schedule to meet the availability of my participants and traveling between two campuses; the investment of time and money paid off. By making the decision to travel to the participants' home campus, I met each woman where they were, both physically and metaphorically. Participants chose the location of the interview to be conducted in their office or another space on their campus, all of which I accommodated. Most interviews took place in the participant's office or a conference room, with one interview conducted in a campus coffee shop. The result was an interview setting that was comfortable and allowed for easy conversation to occur between us, yet allowed for the privacy needed during times when participants shared challenges that have emerged during their career. Some of those caused the participant to become emotional. To underscore the importance of the in-person interview, I do not believe this level of trust could have been established during a phone call or even during an in-person interview held in a large convention center during a national conference. 
For the first interview, I used a voice recorder with the permission of the participants to capture the exchange of information and to provide me with an electronic audio file for later review. In my attempt to support each participant in taking ownership of their story and to assist with confidentiality, participants were asked to choose their own pseudonym at the start of our time together. This process came easily for all and resulted in some choosing a simple initial as their pseudonym, while others chose either a name they liked or a name that held special meaning.

Once the pseudonym was chosen and noted, I provided a prompt to begin the interview. The six women were given the same prompt at the start of our time together - "talk me through your professional story." My reasoning for starting each interview in the same manner was to allow the women to use their own words to convey their experiences. While I did ask clarifying or follow-up questions when necessary, and I attempted to ask all the questions in my interview protocol (Appendix C), I intentionally remained silent for much of both conversations, the first and second interviews. I provided non-verbal cues such as nodding and smiles to show my interest and engagement. There was laughter at times of some interviews, as well as instance of shared emotion when I empathized with a story being shared. All first interviews began in a linear way with how the participants entered the profession. Most worked through their story chronologically, and they referred to their resume when necessary to make points they believed to be significant to their story. In situations where the participant had completed the preinterview homework (the sketch), they used their drawing as starting point in their storytelling.

My preference was to create an interview environment that was less structured, allowing the participants greater control in the process (Riessman, 1993). I utilized an interview protocol for both the first interview (Appendix C) and the second interview (Appendix F) of intentionally 
designed questions created to draw rich, detailed accounts from the participants (Lofland \& Lofland, 1995) regarding their career development and what they have needed to be successful. Aspects of the KCM framework guided the creation of the core list of interview questions that I asked of every participant. Prior to each separate interview, I studied the participant's resume to add questions distinctly applicable to the corresponding participant. I also took notes during the interviews that were reviewed later during data analysis. The result was a semi-structured interview that was well-paced, and the use of open-ended questions produced the narratives I was seeking. Interviews lasted approximately an hour.

I was curious if the topic of gender would emerge naturally through conversation, if it would be talked about specifically by the women, or if I would be the one to bring it up. For these reasons, when designing the interview protocol (Appendix C), I saved specific interview questions about gender for the latter part of the interview. Existing scholarship depicts a chilly climate for women students (Hall \& Sandler, 1982; Salter, 2003) and women faculty (Hart, 2016; Sandler, 1986), corroborating my assumption that the women in this study may not have been socialized to talk about gender in the academic workplace. Four women in this study brought up gender early in our first interview. They appeared to be comfortable approaching the topic and provided concrete examples from their professional stories where gender was either a hindrance or had helped them. For the other two women, I noticed they referenced gender within a few of their comments but never in a direct way. Once I prompted them to discuss gender, they moved easily into the topic. Like their fellow participants, these women shared instances where gender was strongly connected to people, jobs, and situations in their career. In attempting to understand this breakdown in terms of who talked about gender and how, I consider factors like the functional units where these women work, their comments about mentorship and supervision, 
how they define success, and more that will be explored in Chapters 5 and 6. Finally, a key factor that could have influenced how easily the women discussed gender is that I am also a woman, and we had strong rapport, which will also be discussed in Chapter 6.

\section{Sketch}

As noted previously, participants were given a homework assignment to complete prior to the first interview, an activity adapted from Nyquist et al. (1999) in their study of the graduate student experience. Providing their class with paper and pencil, Nyquist and her team instructed participants to use 10 minutes to reflect on their graduate journey thus far, and depict the journey using visuals or text. Findings provided "powerful glimpses into the realities" (Nyquist et al., 1999, p. 18) of the participants. Most interesting to the researchers conducting this exercise was the revelation and amount of negative experiences expressed by the participants. In the end, the activity produced valuable insight into the needs of graduate students, information that could be vitally important to those in positions to enact change.

In the spring semester of 2015, my second semester as a full-time doctoral student, I enrolled in a class called The Professoriate, taught by Dr. Jeni Hart, and participated in the Nyquist et al. (1999) exercise. Like their participants, I used a variety of sketches, colors, and text to depict my higher education experiences. I found the experience quite personal and realized it was the first time someone had asked me to explain my path. Then, we shared our stories with the class. I found that my peers' depictions included similar ups and downs as mine, but I also learned of struggles and opportunities not previously revealed. This exercise resonated with my instinct to understand others' experiences, build rapport, and advocate for change based on shared needs. 
Inclusion of this activity in this study was strategic in that it added a unique element to the narrative inquiry, engaging the participant before the interview even began. The sketch is an example of a participatory visual method (Mannay, 2016) becoming more recognized particularly in narrative research. Andrews, Squire, and Tamboukou (2008) described such an activity as collaborative work. Further supporting my use of the sketches as a data form, the highly regarded qualitative researcher Catherine Riessman (2008) emphasized that words are but one communication form, and urged researchers to carefully study visual materials, including "aesthetic representations" (p. 141). Finally, when asked to tell stories, individuals frequently do so in a way that is not chronological (Creswell, 2013). The sketch activity engaged participants to visually think through their career development in a chronological manner, limiting the likelihood of unintentionally leaving out key experiences.

My original plan for this activity was to have the participants do the activity at the start of our first interview. Further reflection on this plan revealed a couple of challenges. The time each woman takes to draw her professional story could have varied greatly, particularly when asking individuals to reflect on the culminating experiences that led to the midpoint of a professional career. Setting aside time to create the drawing in my presence would have likely created significant delays in the start of the actual interview. I also acknowledge some participants may lack confidence in their artistic ability and may feel uncomfortable drawing with me seated in the same room. However, the positive contributions the drawings made to the interview (and overall study) far outweighed logistical challenges. Thus, having participants draw their story on paper prior to my arrival allowed them to reflect on their professional story in private and to take as much time as they needed. They determined exactly how they wanted to be represented. This step was integral in providing each woman a degree of control in how her story would be told. 


\section{Second Interview}

After completing first interviews and reviewing the transcripts, I e-mailed each transcript to each participant as part of the member checking process (Appendix D). This served as a vital step in establishing credibility in the study (Lincoln \& Guba, 1985) as well as maintaining rapport with my participants. It was important that participants had an opportunity to correct any misinterpretations of what was said during the first interview. No changes were made, but my own review determined that second interviews were necessary to obtain the depth of information needed to answer my research questions. While the first interviews provided comprehensive overviews of everyone's professional story and allowed me to ask questions about specific experiences, I still had unanswered questions. Within the same e-mail with transcripts, I also requested a second interview (Appendix D). Particularly after completing first interviews from all six participants, I noted a few topics brought up by some participants that were not mentioned by others, resulting in my desire to revisit conversations with all participants. Examples of areas where I hoped second interviews would allow us to delve deeper included, but were not limited to: (a) areas in which they are challenged professionally; (b) how prepared the felt entering the student affairs field and what more could be done now; and (c) their perspectives on the Professional Competency Areas for Student Affairs (ACPA/NASPA, 2015). I used this list to create the interview protocol for the second interviews (Appendix F). Since the list of follow-up questions was shorter than the list from our first interview, as well as for the sake of time, I chose to employ a phone interview method for the second interviews. Once second interviews were scheduled, I sent an e-mail reminder (Appendix E) with two attachments - the interview protocol created for the second interviews and an electronic copy of the Professional Competency Areas for Student Affairs Educators (ACPA/NASPA, 2015). Using an app on my smartphone called 
Tape-A-Call, I conducted second interviews with five out of six of my participants. One participant had multiple scheduling issues that prohibited a second interview. I took comprehensive notes during the second interviews, some of which will be referenced in Chapter

6.

\section{Data Analysis}

According to Riessman (2008), narrative analysis may take the direction of the actual telling of the story (structural), to whom the story is intended (dialogic/performance), or what was actually said (themes). For this study, I was interested in the latter. Approaching the data analysis process inductively allowed me to identify patterns in the data and organize themes from the bottom up (Creswell, 2013) rather than forcing data to fit the theoretical framework. Throughout the analysis, I began narrowing down the key moments that defined each participant's experience.

This study relied heavily on participants' perspectives, and how I made meaning of the information shared. True to the narrative inquiry method, the data "need to be analyzed for the story they have to tell, a chronology of unfolding events, and turning points for epiphanies" (Creswell, 2013, p. 189). While this study did not include the participants' entire life story, the chronology of their professional story was of utmost importance. This chronology proved to aid in the analysis portion of my study. While other forms of narrative inquiry result in text that the researcher must arrange and rearrange (Mishler, 1991), I found the task of finding specific information to be quite manageable as I revisited the text multiple times. 


\section{Representation}

When determining my approach to data analysis, specifically given my epistemological perspective as a constructivist, I was guided by Riessman's (1993) five levels of representation in the research process, in which the researcher is engaged in interpreting the narrative.

\section{READING (5)}

ANALYZING (4)

TRANSCRIBING (3)

TELLING (2)

ATTENDING (1)

I was initially a bit overwhelmed by the amount of data collected; it was challenging to determine the right way to tell these stories with accuracy and respect while also attending to my own role in the telling of each narrative. Consciously taking each of the following steps in the order presented allowed me to maintain a sense of direction. Based upon the work of Riessman (1993), I followed the following process:

1. Starting at the attending level allowed me to simply approach the data with the sole purpose of reflecting on the experience as a whole, thinking specifically about my role in the constructed reality.

2. Telling the story in a particular way based not only on the audience, but as influenced by my own personal narrative as a woman (among other characteristics). 
3. Having the audio data transcribed was more than simply producing written text, as I made intentional decisions on what to include that will certainly influence how readers receive the narrative.

4. To analyze the data by pulling apart sections for close examination, comparing sections, and choosing how to tell the story in the final form.

5. Finally, the presentation of the final text for reading by others means they will bring their own perspective.

Although the first four stages were pertinent to the data analysis portion of my study, I found the attending and analyzing levels most significant to my role. In fact, both levels took much more time than I originally planned; however, rushing the process would have negatively affected the findings. I needed the extra time for reflection on the data collection process, specifically for my role in engaging with the participants and their stories. Similarly, each time I revisited the data for additional analysis, I found new information that I immediately wanted to then compare with the other transcripts. How the readers interpret the end story is unknown.

\section{Demographic and Academic Background Information}

The first data source to be analyzed was the information collected through the electronic preinterview questionnaire. The following information was obtained:

- All participants self-identified as women;

- Four participants are within the 30-39 age group; two are within the 40-49 age group;

- Four participants are Caucasian; one is African American, and one is Multiracial;

- Four participants are married; one is single [never married]; one indicated she is single but did not clarify whether she had been previously married; 
- Of the participants who are married, their partners work in the fields of PK12 education, higher education, retail, and as a tradesman; and

- Three participants indicated full-time caregiver responsibilities and have children under the age of 18 .

\section{Resume}

The second data source to be analyzed were the resumes e-mailed to me by each participant. I printed a hard copy version of all six resumes for my review. The following information was obtained:

- Participants earned undergraduate degrees in a variety of disciplines including communication, journalism, exercise science and recreation, English, and two from psychology;

- Four participants earned master's degrees related to higher education, the other two from different disciplines—recreation and American studies;

- One participant has earned a doctoral degree in educational leadership, one is in the process of completing a similar degree, and two others have taken some doctoral level courses.

\section{Interviews}

Transcribing the interviews was a key element of data analysis. For the first interview, the transcription was verbatim, apart from pseudonyms used for participants. The transcript included all utterances (Levinson, 2000) by both the participant and myself as the researcher, defined by Gee (2011) as words or phrases with a variety of possible meanings. Most of these were non-lexical utterances such as "uh huh," "mmmm," "yeah," or short words such as "okay", "sure", and "really?" to indicate my interest in the participant's story and to encourage her to 
continue. As previously mentioned, attention was directed to the turning points within the individual story, the crucial elements, as well as quotable statements. Although I considered transcribing the interview myself, I later chose to use a transcription service to assist with accuracy and efficiency. I found that I was still able to immerse myself in the stories by taking the following steps:

1. Listening to the audio recordings of each interview immediately after the interview concluded;

2. Reading through each transcript multiple times and making notes; and

3. Comparing notes I made on the transcripts with the notes in my own journal.

This method of verbatim transcription resulted in transcripts that varied in length between 14 and 22 single-spaced pages, with the average page length of approximately 18 pages. Each transcript was e-mailed to the corresponding participant for review. I asked participants to check for specific errors in spelling as well as errors in how they believe their stories were represented. In addition, I asked them to indicate any clarifying information they felt would be important to add or that they perhaps unintentionally left out the first time. None of the six participants indicated changes to the transcript they received. I reiterated my use of pseudonyms to protect their identity and none of the participants indicated concern in this area. Although the e-mail exchanges and multiple transcript reviews took a significant amount of time, these steps enabled me to feel immersed in their stories from start to finish.

I chose not to have the second interviews transcribed. The first interviews were long and detailed, as the participants talked through years of professional experiences, requiring many read-throughs to find the details I needed to fully understand their experience. The sole purpose of second interviews was to ask specific follow-up questions that were not particularly related to 
one another. Therefore, I did not deem it necessary to produce a second transcript for each participant. Seeking validation for this decision, I referred to Patton (2002):

When a qualitative research design involves the collection of audiotaped in-depth interviews or focus groups, researchers must decide whether their analysis is best supported by transcription or by researchers' notes derived from or supplemented by a review of the audiotapes. (p. 380)

I did not include second interview quotes in the study, but rather relied on the notes I took during second interviews, and replayed the audio files when additional clarification was needed.

\section{Sketch}

Since both the interview transcripts and the sketch complement one another, they were analyzed together as one unit for each participant. Although all participants were asked to complete the sketch activity prior to my arrival for our first interview, only three chose to do so. The other three admitted to reading the Nyquist et al. (1999) article prior to the first interview; two simply did not have time to complete the sketch and the third stated she appreciated the article but felt she would be able to tell her story apart from the sketch. For the three participants who completed the sketch, I was pleased with their attention to detail and enthusiasm in sharing their work. When I arrived to the interview with these women, the sketch was displayed and ready to be incorporated into our discussion. What resulted were meaningful stories depicted through vibrant colors and unique shapes and symbols that explained the opportunities, challenges, and unknowns of a professional story.

\section{Thematic Narrative Analysis}

Once all data were collected, edited, and the transcripts approved by participants, I spent significant time reading through each datum multiple times. Consistent with the goal of thematic 
narrative analysis, subsequent readthroughs allowed me to look for commonalities among the six stories. To establish a process for examining my data, I referred to the data analysis and representation recommendations for narrative analysis provided by Creswell (2013):

1. Read through text and make notes to form initial codes;

2. Describe the story or objective set of experiences and place in a chronology;

3. Identify stories and locate epiphanies; and

4. Interpret larger meaning of the story.

I used inductive coding to incorporate immersion in the details and specifics of the data to discover the important categories, dimensions, and interrelationships (Patton, 1990). The first readthrough highlighted points of interest, particularly those I noted in my journaling. Comparing each transcript with its corresponding resume and sketch (where available) provided both a big picture view of each participant's story as well as the significant details. During the second reviews, I began to make notes of what was highlighted to form initial codes, one or two word descriptions of what I had noticed. Subsequent reviews and comparisons helped me to get to know each participant and their story better and to ensure I understood the information conveyed by them all. I often refined the name of the codes to best reflect my understanding of the material. I initially planned to use a web application called Dedoose to upload the transcripts and formulate codes. Although the program offers functions to make certain steps of data analysis easier, the resumes and sketches would not have been easy to upload, adding challenge to my desire to compare the data. I realized my preference was to code by hand with hard copy printouts of all data sources.

Once all transcripts were reviewed and coded, I created an Excel spreadsheet to organize the codes, with each participant having their own column. When I believed I had a strong 
understanding of a participant's story, I reviewed all of my notes and entered all possible codes into the corresponding column. Then I followed the same process for the rest of the participants. The lists were long, but comprehensive, with the average of 32 initial codes per participant: $\mathrm{E}=$ 29; $\mathrm{J}=30$; Cathy $=30 ;$ Anastasia $=37$; Betty $=33$; and $Z o e=34$. The spreadsheet condensed the codes into a visually appealing format. This simplified the identification of repeated subject matter (Phoenix, 2008).

Since all participants were asked the same questions, perhaps I should not have been so surprised to note the use of similar language by some participants. Reviewing the table, I searched for patterns of similarity, as well as patterns of difference (Daiute, 2014). For example, codes like gender, supervisor, and professional development appeared on each participant's list of codes. Aside from the similarities, I was careful to attend to their distinguishing differences. This awareness allowed me to go beyond the identification of themes to "analyze their intersection... and illuminate the human experience" (Josselson, 2011, p. 240). Mindful attention to what can be learned from personal narratives served as an authentic approach to meaning making, increasing this study's potential to influence others.

\section{Use of Theoretical Framework in the Research Process}

I first used the KCM to inform the creation of the interview questions. While I felt strongly about a few interview questions that would have been included in the study no matter the choice of framework, other questions were linked to the KCM's parameters of authenticity, balance, and challenge. Examples include, but are not limited to, questions about how the women define success and what they enjoy most about their jobs (i.e, authenticity); how they approach work/life balance and seek support (i.e., balance); and how they determine areas for growth (i.e., challenge). An intentional choice was made not to consult the KCM during the data collection or 
initial analysis, instead preferring to use inductive coding so that themes emerged naturally. My concern in using the $\mathrm{KCM}$ throughout was the risk of deductively coding the data and attempting to fit the data into the framework.

In Chapter 4, I present a summary of the six individual stories to provide readers with a better understanding of the participants in this study. In Chapter 5, I present the findings from the study. Also included in Chapter 5 is a section where the findings are interpreted through the KCM. Specifically, I note which themes relate to the KCM parameters. I also note any overlap, meaning some themes related to more than one parameter, while other themes did not correspond at all. While the theoretical framework was not used to establish the codes or themes, consulting the framework after data analysis helped make meaning of the participants' experiences through their career development through the perspective of the kaleidoscope career.

\section{Trustworthiness}

Throughout the data collection and analysis, I reminded myself of the questions posed by Pinnegar and Daynes (2007): Who owns the story? Who can tell it? Who can change it? The stories I collected through this project do not belong to me, and I remained mindful of this as I listened, posed questions, identified themes, and present findings. To further enhance the trustworthiness of this study, I employed multiple techniques, including triangulation methods of peer review, clarifying researcher bias, thick, rich description, and member checking (Creswell, 2013). Finally, data saturation was used as a means of validating the research process used for this study.

To adhere to the peer review method of triangulation, once data were gathered and coded, I debriefed and reviewed the entire data collection and analysis process. Lincoln and Guba (1985) defined the role of the peer reviewer as one who keeps the researcher honest, challenges 
the researcher's assumptions, and asks hard questions about methods and interpretations. My doctoral advisor was an appropriate choice for peer review, as she is familiar with the phenomenon being studied, the context of the study, and provided an appropriate balance of challenge and support.

Throughout the study, I attempted to remain honest and transparent about my biases and assumptions related to the study and its anticipated findings. As mentioned in my positionality statement, my previous work and academic experiences within the field of student affairs brings a close familiarity to the topics being explored, as well my own shared identity with the participants. Likewise, the choice of narrative research is autobiographical; "our research interests come out of our own narratives of experience and shape our narrative inquiry plotlines" (Clandinin \& Connelly, 2000, p. 121). Underscoring the importance of reflexivity as an interpretive standard of conducting qualitative research (Creswell, 2013), my researcher positionality statement is written candidly, and participants were provided with purpose of the study prior to data collection.

Rich, thick description should be detailed and context-sensitive (Geertz, 1973) in relaying the information I collected to those who read the final product. Potential readers of the study will most likely represent different aspects of the student affairs field. No matter their professional title or stage of career development, it is important that they can fully understand the findings to determine transferability. I have provided details in my descriptions of participants' stories, including interconnected details (Stake, 2010). A goal of this study to is to provide detailed insight that will benefit other student affairs professionals. As Erlandson (1993) described, the reader determines if findings can be transferred when they have shared characteristics. This is most easily accomplished when the data are described in depth. 
The use of member checking attempted to ensure each participant's voice is represented accurately. Described as "the most crucial technique for establishing credibility" (Lincoln \& Guba, 1985, p. 314) in a study, the technique allowed participants in the study to review and confirm the credibility of the information represented in the narrative. For this study, the process entailed e-mailing a copy of the interview transcript to each participant, asking for clarification or corrections. For many reasons, it is important to me that my study participants felt their voices were fully heard and respected, and that I represented them in full accuracy and authenticity. Therefore, the member checking technique gave participants an opportunity to correct errors, volunteer new information, and challenge any perceived misinterpretations.

Finally, careful steps were taken to ensure the research design is appropriate for the study, including all aspects related to data collection and data analysis. Regarding data collection, data saturation is not completely about numbers, but rather depth, and neither a large nor small sample size guarantees one will reach data saturation (Burmeister \& Aitken, 2012). The selection of the sample size is specific to individual studies, and I stated previously how I reached a final number of six participants. Regarding data analysis, the process was thorough to the point of exhausting all data collected. Data saturation occurred when I no longer found new themes in my analysis.

\section{Research Ethics}

I have been committed to practicing ethical research throughout the entire study, a study that has little to no risk for participants. While a couple participants appeared to experience minor emotional upset when discussing specific experiences or examples from their professional career, there were no foreseeable risks associated with the study. 
A misconception of qualitative research is that ethical issues only arise during data collection (Creswell, 2013). My first step of gaining approval from the University of Missouri Institutional Review Board (IRB) was required to proceed with data collection. Participants were provided a written consent form that explained the purposes and procedures of the study, and that their participation was confidential and voluntary. Participants were given the right to withdraw from the study at any time, but none made that decision. However, despite continued interest in the study, multiple attempts to reschedule the second interview with one of the participants were made and cancelled due to personal or professional conflicts.

Signatures on the consent form were not required, as they would negate the confidentiality agreement by asking participants to disclose their name. Participants agreed to participate in the study confidentially, meaning their actual names and/or institutions will remain private, and pseudonyms were used throughout the study. All hard copies of data collected (e.g., resumes, sketches, transcripts, audio files, and notes) were stored in a locked file cabinet and will remain there for 7 years after the study is completed, adhering with university policy.

\section{Limitations}

Taking note from Daiute (2014), even the most careful narrative research design must consider the impact values have in organizing narratives. Thus, my epistemology and life experiences were taken into consideration throughout the study. The social constructionist perspective is that all narratives "sit at the intersection of history, biography, and society" (Liamputtong \& Ezzy, 2005, p. 132). I am a woman studying women. I am a former midlevel student affairs practitioner studying current midlevel student affair practitioners, most of whom I know on both a personal and professional level. Even the co-construction of meaning raises issues of dependability, and whether I could reasonably represent the study's participants. 
Through my researcher positionality statement, I endeavored to provide transparency regarding my motives and personal connections to the topic, participants, and intended use of findings.

Another limitation stems from the complexities of interpreting the narratives of others. Narrative inquiry begins with the expression of lived and told stories. Where other qualitative designs may provide more direction in terms of starting and ending points, narrative research is different (Squire, Andrews, \& Tamboukou, 2008). Using the technique of data saturation signaled to me when to stop the data collection process. "Data saturation is reached when there is enough information to replicate the study, when the ability to obtain additional new information has been attained, and when further coding is no longer feasible" (Fusch \& Ness, 2015, p. 1408).

\section{Summary}

The choice of narrative inquiry to explore the careers of women, midlevel student affairs practitioners fits for many reasons listed in this chapter. Perhaps most significant is the connections between my epistemology, the population to be studied, and my personal identity. Chase (2005) identified five interconnected, analytic lenses used in narrative inquiry. But it is his second and third lenses that resonated with my approach to this study. The second lens described the ways in which individuals convey thoughts, feelings and behavior through narrative (Hunter, 2010). Regarding this study, interpreting the themes through the KCM as a theoretical framework attempted to use this lens to better understand the career experiences of my participants, as told through their narratives. The third lens described the ways in which those narratives "were constrained and influenced by the social mores of the era" (Hunter, 2010, p. 47). My intentional focus on women at mid-career attempts to use this lens to better understand the role gender plays in the student affairs careers. 
Next, each of the six women's narratives is explored separately in Chapter 4. While my primary interest has always been the meta-narrative that emerged from comparing their stories, I am equally committed to the preservation of each individual story. Each story stands alone in terms of personality, career path, and career decisions. Each story also presents details that related to the larger themes strongly evident in the data and relates back to the aims of the research. 


\section{Chapter 4: Presentation of Individual Narratives}

I consider it a privilege that six women shared with me their professional stories. More than a resume or summary of experiences, each woman carefully walked me through their career path, including the highs and the lows. The experience was incredibly personal for them and I am honored to have been welcomed so openly into portions of their story that may otherwise have remained private. Some aspects of their stories included details related to relationships, family, and health. They have all benefitted from strong woman mentors, and a few mentioned mentors who are men. We discussed important decisions like moving closer to family, moving away from family, or decisions to — or not to — start their own families. Experiences of racism, ageism, and discrimination based on gender and sexual identity were shared. They provided examples of colleagues and supervisors who were supportive mentors and instrumental in their development. They also provided examples of colleagues and supervisors who were unsupportive, critical, and even made them question their value. Finally, they seem to be in agreement regarding what the student affairs field should be doing to better prepare new practitioners. These examples, and other themes, will be discussed in Chapter 5.

My attempt to capture the overall story of these women also allowed for me to highlight the unique components of their individual stories. As they described themselves, their stories were predictable at times, following a storyline that Polkinghorne (2005) described as having a beginning-middle-end structure. This style was unsurprising to me, as constructing stories in this chronological way is tempting when asked to talk through several years of a person's life. But Phoenix (2008) noted an advantage of allowing the narrative to take different directions, including a focus on both big and small stories included in the narrative. 
Once the interviews evolved into natural conversation, information then emerged nonsequentially. These turning points signified to me that the women and I had moved beyond building rapport, progressing into a deeper level of trust. This evolution allowed them to revisit situations they may not have thought about in quite some time. The ability to view such a situation with hindsight and maturity often brought new perspective and even new details to light, allowing me to provide some interpretation of the information I heard. Some of my questions caused the women to consider aspects of their career they previously had not. When such a situation occurred, I allowed time for reflection (and sometimes emotion) as an outward sign of respect to the participants.

This chapter introduces the women and provides an overview of their background and story. Each participant in this study was given the opportunity to choose their own pseudonym. At the time of participating in the study, each woman self-identified as a woman midlevel student affairs practitioner. Three participants completed the sketches as part of the pre-interview homework activity, and where applicable, snapshots of these sketches are included as visual data in Chapter 5. Below, the participants are listed in the order they were interviewed for this study.

\section{Differences and Commonalities}

A review of the data collected from the pre-interview questionnaire, six resumes, three sketches, six interview transcripts, and five audio files highlights several similarities among this group of women, but also unique aspects. As a reminder, four participants work at the same institution, Flagship University, and the other two participants work at Midwestern University. Working in the same environment may naturally create similarities in professional development opportunities, advancement opportunities, and organizational climate. Each woman represents a different functional unit within student affairs, although two women work in offices that have 
recently been moved out of the organizational structure of student affairs even though their work still strongly overlaps with the remaining student affairs offices. Some of the women attend different annual professional conferences, and some have chosen to step away from the traditional student affairs organizations. As noted in Chapter 3, some of the women have partners and caregiving responsibilities while others do not. The differences between individual stories add to the potential transferability of this study's findings, as no career is the same as another. However, it is the similarities in their stories that provided direction in interpreting the findings.

While the meta-narrative of the women's stories is the primary interest of this study (presented in the next chapter), there is value in the individual narratives. Learning the unique aspects of each woman's professional story increased my ability to fully answer the research questions that guide this study. Each story deepened my understanding of the research problem, the sacrifices and perseverance, opportunities and strategy that enabled the women to progress to the midlevel career point. Each story began to build upon the one before, contributing to shared concepts, while retaining their identities.

There exist strong parallels between the women in terms of how they entered the field, the strategic decisions they have made at various career points, and how they reflect on their stories. A couple similarities were almost uncanny. An example is when asked to share the personal attributes they believe have assisted in their career development, all six women mentioned StrengthsQuest, a web-based tool that uses a positive psychology approach that helps individuals identify the top five strengths most descriptive of themselves (Clifton \& Anderson, 2001). Each woman first asked if I was familiar with the strengths approach, then proceeded to list two or three to help answer the question. This example surprised me but then provided an unanticipated link between the women. Additional themes identified between the narratives 
eventually lead to the larger themes that are explored in detail within Chapter 5, as well as the discussion in Chapter 6.

\section{Participant \#1: Ann}

Ann is an associate director at Flagship University where she is part of the senior leadership team of a student affairs department focused on student wellness, and is 7 years postmaster's degree. Earning an undergraduate degree in communications from Flagship, Ann worked for a short time outside of higher education, but remained in the same town. Missing the student engagement that she enjoyed during her own student experience, she sought the advice of an academic mentor still on campus, a woman faculty member. This conversation led to meeting with a different woman faculty member, and solidified Ann's decision to pursue student affairs as a career. She negotiated an arrangement that allowed her to complete her master's degree in higher education administration while working full-time to gain additional experience. The decision to return to Flagship was also influenced by her personal life at the time, as her partner lived in the same town as the college. As she continued to be offered opportunities to increase responsibility, Ann's commitment to the department and her supervisor strengthened. By expanding her skillset, she also earned promotions. Her resume reveals an individual committed to the morale, leadership potential, and over health and wellness of students on her campus.

Ann appears to be intentional in how she approaches professional development. She participates in numerous trainings and committees both on and off campus, and has served as a consultant for other institutions. While several opportunities have been offered to her because her supervisor saw her potential, other instances have been more strategic on Ann's part. She shares several examples of times she has candidly asked to be part of conversations and meetings to learn new information or "diversify" her skillset. A specific example where Ann showed 
initiative simultaneously highlighted gender issues in her department. There are some job skills typically held by men in her department; however, this has not prohibited Ann from expressing interest in learning these skills. As a whole, though, gender has played a positive role in her professional story regarding her mentor from her undergraduate years and her current supervisor. Ann describes her work environment as "atypical" in that the leadership in her department is majority women and emphasizes the experience has been constructive. However, she notes this gender dynamic appears different than the rest of her campus.

Consistently thinking about the bigger picture in terms of her department and her career, Ann believes professionals should "own where the gaps are." She observes the student affairs field has changed in terms of size and requirements needed for career advancement and mentioned several times the need for professionals to "advocate" for themselves rather than waiting on opportunities to unfold. She believes the field could have better prepared her to "collaborate in ways...to use all resources" and skills to gain a "higher level of buy-in" from campus partners. Ann ponders if current midlevel professionals are as prepared to move into administrative roles as our predecessors. She ties this concern to the dichotomy of how much the field as grown, while also requiring professionals to have specific experiences and skills.

Ann possesses inherent skills like adaptability and an ability to "arrange and put things in order" and has incorporated these into her professional life. She is getting better about knowing her "capacity" so that she can limit saying yes to "one more thing." This latter point is more important now that she is married and has a young family. Attempting to strike balance between work and home, she enlists communication as a strategy, but is honest in that it takes effort to keep those "boundaries" intact. An example is checking e-mail late at night or taking phone calls 
from students on the weekend. When this occurs, she and her partner often need to "recommit to whatever decision" they have made in terms of protecting their family time at home.

The aspects of her job that are most meaningful to her are the relationships with her colleagues and direct supervisor, her enjoyment in coaching students, and using her knowledge of student development to assist her colleagues in navigating student issues. Although Ann is loyal to her supervisor, she is not afraid to "test the waters to see where else [she] could fit" and she does this by applying to at least one new position each year. She intentionally involves herself in activities outside of her functional unit so as not to appear "pigeonholed." The process of applying to other jobs provides her insight to how others perceive her experiences. She also feels "validated" that her efforts are worthwhile and are preparing her to do other things, and makes her "more confident that she could do something different." She is honest with her supervisor about this annual process and has found her to be "very supportive." Ann personally equates success to feeling accomplished and at the end of the day, and wants to feel she "had an impact" on others. At this point, her student affairs career has unfolded solely at Flagship and she is content in her current role, but expressed interest in being a "VP of a small institution" as a long-term professional goal.

\section{Participant \#2: Liana}

Liana is a senior coordinator at Flagship University, where she directs a student affairs office, and is 11 years post-master's degree. Initially interested in the psychology field, Liana was introduced to student affairs as a career option during her senior year of college. A woman colleague at another institution explained the graduate degree that would provide a pipeline into working with college students. Liana earned her master's degree in higher education and approached the job search with an open perspective. She shares this part of her story with a bit of 
humor in that she was "kind of flying by the seat of [her] pants." An opportunity presented itself that would also allow her to move to the state where her partner resided, thus beginning her career at Flagship University. Her resume showcases experiences related to student involvement, engagement, responsibility, and integrity.

Liana's decision to continue working at Flagship allowed her partner to pursue a career simultaneously in the same geographic area. While they were initially open to moving away from their careers at some point in the future, their decision to start a family and the success of both careers strengthened their ties to the Flagship campus community. While it was higher education professionals who helped guide Liana's early career decisions, she has since expanded her mentor network and finds it beneficial to consult with them as she navigates change. At some point in her story, Liana's decisions expanded beyond her own career and future and she enjoys being "a champion" for her partner's career, as her partner has always supported hers.

When asked what keeps her engaged in the field, Liana is honest in that she has consistently been encouraged to try different things - teaching, make programmatic changes, and supervising - to name a few. Increased responsibilities continued until a major job shift 3 years ago, that was out of her control. This unexpected job change that was not initiated by Liana was a difficult point in her career, but has resulted in a surprising outcome. The result of the shift has been positive in terms of taking ownership over a new functional unit, one that she had not considered previously. The shift also provided opportunities to reflect on the different stages of her career up to this point, specifically the influence work had on her personal life. The new job is more contained to business hours, has a trusting supervisor, and allows her to "be present" with her young children and partner. She has learned to prioritize better for personal time and her 
partner and kids' schedules and takes more ownership in being away from e-mail on nights and weekends and enjoys greater autonomy.

Liana enjoys being "challenged on a daily basis" in her current role and finds her work "validating." Her responsibilities align with her personality in that she "has always been one to believe in people." The salience of gender in the workplace is evident through Liana's interactions with individuals through her position at the university. Liana shared examples of how she has been talked to or treated because of her gender, but claims she feels comfortable navigating these difficult conversations. Liana has learned to "manage up" differently and has developed solid rapport with colleagues and upper administration, a skill she attributes to developing a different level of confidence. Liana's approach to supervising others has been largely shaped by her own supervisors, both previous and current. She noted several instances where her supervisor's philosophies and actions did not align, and the associated ramifications.

Considering what skills and abilities the student affairs field could have emphasized stronger in her professional preparation, Liana categorizes the list under the title of "administrative functions" including supervision of staff, campus politics, assessment, budgets, and how each functional unit "connects to the bigger picture." Years of experience have showed the potential benefit of incorporating "perspectives from other disciplines" into the student affairs work, specifically organizational analysis. Equating success to happiness is Liana's approach to her career. She enjoys the moment and rather than focusing on an end goal, she is energized by constantly looking at the next step in front of her, both personally and professionally. Liana does not make decisions if she is unable to know the "why" behind them. At this point, Flagship has been the location of her student affairs career and she is content with her "overall journey." 


\section{Participant \#3: Cathy}

Cathy works at Midwest University as an assistant director in an office focused on inclusion, and is 5 years post master's degree. Midwest is a common thread woven through her professional story. Although she completed both her undergraduate and graduate degrees (journalism and higher education administration, respectively) at the institution, Cathy left for several years and was employed full-time between degrees, inside and outside of higher education. This sequencing, although unintentional, brought maturity and perspective to her career. Some job changes were made based on personal preference while others were made with a partner or to be closer to family.

When comparing her various work experiences, Cathy possesses a keen ability to identify consistent skills that contributed to her success in each role. Examples include communication skills, teaching, and helping people grow. An approach from another field that she has incorporated into her student affairs work is that of "asking versus telling" as a communication strategy with her staff. But what she finds the most meaningful about her work is mentorship. Throughout her career path, she has always had someone in her life who understands her values and encourages her to do what is best for her at that time. Her definition of success is linked to "internal feelings" and something she can "quantify as positive change."

In terms of professional growth, Cathy's approach is to look at the calendar and identify what "scares" her or what she is not looking forward to, and then to "generally lean in." She attributes the courage this takes to the way she was raised and that her parents cared more about her "effort than results." This tactic has enabled her to learn more areas the field did not prepare her enough for, like budgets. Another way Cathy believes the field could better prepare 
professionals is to "listen to their soul" when job searching instead of searching for the perfect institution, or the ideal supervisor, or even just taking the first job that becomes available.

Rather than being heavily involved in professional associations as a way of growing professionally, Cathy takes advantage of webinars that she can do on her own time as she embraces lifelong learning. Cathy has also adopted a more direct approach structured as "one-onone" learning. Time after time, the approach pays off — she invites people to coffee to learn more about their story, resulting in new connections. She shares these experiences with the staff she now supervises as an encouragement for them to reach out for intentional connections and believes in "paying it forward."

She is also cognizant of the personal toll that her work can take. Cathy begins every individual meeting with her staff the same way, by asking them to share how they are doing"how are you and what's going on in your life?" She acknowledges that working in this field means she often spends more time with her co-workers than she gets to spend with loved ones, and living far from her traditional family means she has created a "family-like life... with friends and colleagues." However, a strategy that Cathy uses to separate her work and personal life is to "turn off e-mail in the evenings," a practice her colleagues and staff are aware of and try to respect. She sets aside half an hour each morning to review e-mails and makes weekly to-do lists, to stay organized. Cathy also employs healthy strategies that include taking frequent opportunities to "get up from [her] desk and take a 10-minute walk" on campus. During these walks, usually between meetings, she enjoys the quiet or puts on headphones.

The influence of gender roles has played a positive role in Cathy's career in terms of mentorship and supervisors but recognizes that not "all women need to be nurturing." That is not her expectation. Cathy's commitment to issues of justice and equity, policy work, and her desire 
to be in a "helping field" keep her in student affairs. In her words, "it is really all about the people... and that is consistent through my story." Cathy hopes that the field will begin putting more funding into things they claim are important, and she is interested in getting into more policy work.

At this point, her student affairs career has spanned two vastly different institutions, one being Midwest and the other in a coastal region. Cathy admits that she has "made some moves that make a lot of sense and moves that haven't made a lot of sense on paper but have made sense [to her].” Being open and willing to try new things has allowed her to have experiences like writing her own job description. Cathy does not feel anxious about where her career is headed and is content where she is currently, but is always open to new opportunities. She feels empowered that she is positively contributing something through the work she does and hopes that she always "gets to work in some kids of equity work" whether that be in higher education or elsewhere.

\section{Participant \#4: Anastasia}

Anastasia is the director of an office at Midwest University, and is 13 years post-master's degree. She earned an undergraduate degree in social sciences and a graduate degree in student personnel from different institutions. Having greatly benefitted from the support of two instrumental women mentors, one present through each of her educational journeys, Anastasia credits their involvement for her desire to "give back" by helping students in some way. Recognizing these mentors had master's degrees in counseling provided Anastasia a direction to pursue. After graduate school, Anastasia worked briefly outside the field of higher education but the experience was difficult. She immediately transitioned into a role at Midwest as a new professional, worked her way up, and now serves as the lead administrator for the same office. 
Not one to shy away from difficult work, though, Anastasia quickly "learned quite a few things about how others perceive the work" she and her colleagues do on campus. A problematic supervisor combined with staff turnover signaled the start of the most challenging part of Anastasia's professional story. She was the recipient of damaging remarks by the supervisor and began questioning if student affairs was indeed the best career field for her. Finding a support network through a professional association allowed her to cultivate relationships with colleagues at different institutions, and to see that the field was "bigger than the bubble" she currently worked. A new supervisor who was much more encouraging and an additional professional development opportunity that she found "truly transformative" changed the course of her life both personally and professionally. Equipped with "new language and new lens" solidified that she was in the right place.

As she began to see cultural shifts at Midwest, Anastasia also made the decision to begin doctoral coursework in an educational leadership and policy Ph.D. program at her institution. She has made steady progress in the program but describes the work as "pushing the boulder up a hill." Pursuing the degree while simultaneously obtaining additional roles and responsibilities at work brings new challenges. But, Anastasia can appreciate the changes and reorganizations that have lead others to place her in the leadership position she has rightfully earned.

Acknowledging that her work is difficult, Anastasia "continues to jump back" to the two instrumental mentors who have consistently offered support during each step of her journey. But she has intentionally "sought different types of mentors," specifically a man who has been in the field for a long time and women of color. Acknowledging and "owning" the various aspects of her identity (e.g., race, gender, first generation college student) has been critically important to Anastasia as a person and a professional. Connecting with women who can relate to her 
experience has been helpful in how she thinks about how she is "allowed to show up" within the context of a "boys club," and also how she is "perceived on campus as an advocate for students."

She attends a particular conference annually that reminds her that she is "not alone" in doing the work. Her long-term partner plays an instrumental role in understanding the work and supporting her personally with his unwavering belief in her as a human. She has created systems at work that have allowed her to adopt healthier strategies for self-care. Examples are trying not to check e-mails when off-campus, blocking an hour for lunch on her calendar, and modeling behavior for her staff. Setting "strict boundaries" also allows her to decompress when she needs to process experiences.

A common thread through Anastasia's story is that she finds meaning in what she does, coinciding with her personal values when she can "create access and space for people with marginalized voices." She believes the field could have better prepared her in the personnel area and "navigating the landmines." Moving forward, Anastasia is hopeful student affairs and higher education will soon "move past diversity and focus on equity," mainly how to apply social justice to the rest of the work happening on campus. Defining success is difficult for her because of being a self-proclaimed "perfectionist" and always feels she "should be doing more." But she tries to hold onto small victories, and following the advice of a mentor, she strives to do her "work well and people will notice," which seems to be happening at this point.

Midwest has been the location of her student affairs career and her hard work and loyalty has clearly paid off. Her long-term, end goal of being a chief diversity officer seems quite feasible although she refers to the commonly known imposter syndrome as both "terrifying" and "exciting." Embracing the reality that there is still a lot of work to be done in her area of campus, 
Anastasia believes she has "an obligation, but it's one [she] gladly accepts to pay back forward," which is that people helped her and she wants to help others.

\section{Participant \#5: Betty}

Betty is a senior associate director who oversees several departments at Flagship University, with 2 decades of professional experience post-master's degree. Like many student affairs professionals, Betty transitioned into a career path in higher education first by way of being an involved student during her undergraduate years. After first working primarily in a department focused on student health and wellness, her first professional job formally introduced her to the option of a graduate degree in college student personnel. Once she identified that her end goal was to be in administration, she had a clearer vision of what it would take to get there.

Much of Betty's career has evolved as supervisors saw her potential and offered new opportunities. This progression has certainly paid off in terms of expanding her skillset, helping her become a self-described "generalist" regarding the diverse services that now fall under student affairs. But Betty has also had to advocate for herself many times and is reflective on aspects of her professional life that she has learned on her own. "Nobody taught [me] the difference between advising and supervising," for example. Reflecting on her current supervisor, Betty also notes differences in how some administrators view graduate students and entry-level professionals in student affairs. For example, her supervisor has difficulty understanding some of the expectations new professionals have about job responsibilities, telling Betty "when I was in student affairs and starting out, I wanted all this experience and I didn't worry about hours and I didn't worry about being paid for taking on extra duties.” Aligning her work philosophy more with these new professionals, Betty finds herself in a place where she must advocate on their behalf. 
The influence of gender roles has played an interesting role in Betty's career. She has benefitted from working under a strong woman administrator in a field predominately dominated by men, but has also experienced women who were "not as welcoming" or did not demonstrate actionable follow-through when stating support of other women. Her supervisors who were men have asked her to take on more responsibilities because they believed in her, but in other instances, displayed behavior that conflicted with their words - perhaps stating they were supportive of her personal life and time needed to finish the Ph.D., yet still demanded the highest level of work. Betty has figured out ways to navigate gendered situations and to use gender differences as a strength.

On the personal side, Betty balances a work schedule that is opposite of her partner's. She has his unwavering support but, based on circumstances, she makes important adjustments to her schedule to absorb parenting duties several days of the week. Betty knows her limitations as balance "looks different for everybody," and she uses her experiences to guide conversations with women she supervises as they seek her input. Betty finds meaning in her work because of the "joy (she) finds in doing something new." As much as she enjoys routine, she is energized by working on different projects and lists ideation as a top strength. She enjoys seeing staff grow, and notes that she experiences growth in the process, describing it as "reciprocal relationship that is very engaging."

Mentors have played an integral role in Betty's professional story, including faculty and administrators from inside and outside her institution. One woman in the field supervised Betty as an undergraduate student and still provides insight to this day. Other times, however, mentorship and support emerged from unexpected places. As Betty reached a crucial point in her doctoral journey, deadlines to finish quickly approached. It was her colleagues and staff 
members who "rallied around [her]" in ways that we evident at the time, but also in ways that she only found out much later. Betty expresses great gratitude for their support.

Throughout her story there are examples of sacrifice, whether working two jobs for the sole purpose of learning new skills or taking a chance on a position when there were red flags during the interview process. Other changes were made to support her decision to start a family and to accommodate needs of extended family. Each time, Betty sought perspective from others but mostly went with her internal feelings about the situation. Each time, it worked out. Betty has redefined the definition of success for herself. Rather than focus on how her career is viewed by others, she is more interested in the impact her work has on students. Although Betty has worked at two different institutions, most her professional experiences have been at Flagship. Career decisions are made not only with Betty's professional goals in mind, but also considering her partner and young family. While happy in her current role, she continues progressing towards her goal of being a Vice Chancellor or Vice President.

\section{Participant \#6: Zoe}

Zoe works at the assistant director level at Flagship University with 2 decades of professional experience in higher education. Her academic background includes American Studies, English, Women's Studies, and some doctoral coursework in higher education. Various positions led her to "fall into student affairs" but it is her strong work ethic, intellectual curiosity, and family foundation that contribute to her professional success. As a "fifth generation college educated" woman, Zoe has been greatly influenced by family members who obtained degrees and believes there existed "no option not to go into education." Though her professional story on paper takes different turns, Zoe's explains how each move was calculated. 
Zoe's professional experiences were at two different institutions, but she also obtained knowledge from working with institutions in the same area as where she was employed. She has worked on the coast and now in the Midwest, two geographical areas of the country that are quite different. With much of her background in urban areas, working at Flagship brought new challenges in terms of her identity, specifically the aspects of gender and race. The intersectionality of Zoe's social identity is integral to both her personal and professional life, and her career includes both positive and challenging examples of how her identity has been perceived by others. Zoe notes heteronormative and gendered behaviors exist in her professional story, even in her current role. She "chooses how to present [herself] the moment she walks in the door." Though she attempts to "find humor where [she] can" when helping students learn and grow, interactions with others can look much different. She has been the only woman of color in a class of students, experiences comments about her hair, is asked about her relationship status, and once encountered a woman faculty member who was also a woman of color who discouraged her academic pursuits.

As her career has unfolded, Zoe has relied on her "strong support network" when making decisions. Though she does not have a specific individual who serves as a mentor, she has established a group of people from various backgrounds that she can engage in conversation about different topics. Even at Flagship she has found support in collaborating with key individuals who understand and support her experiences, and offer encouragement.

Recognizing that the definition of success "changes on a very personal level" for everyone, Zoe explains that her "version of success" has changed over time. Moving away from finances and titles carrying the most weight, her social maturity and meeting her established goals has been more enjoyable. She has been intentional in building a diverse skillset, which has 
allowed her to view higher education from multiple perspectives, including the business and financial side. Some of the most challenging work tasks she has been given have resulted in learning more about managing budgets; working with non-traditional students; program planning; and navigating campus leadership, as well as attorneys and realtors outside the institution. Consistently, though, she advocated for the "student experience." An activist who is passionate about social justice issues, she finds meaning in her work when she can "see students stop and think." Zoe values skills like critical thinking and communication skills but believes the student affairs field should prepare practitioners with more "practical skills" and put more effort into helping them bridge theory to practice.

Coming from a "culture of extreme conscious dialogue," Zoe feels frustrated by colleagues and supervisors who struggle to take "action" and instead continue to simply discuss students and situations. In such situations, she carefully considers how to "negotiate" and "engage" so that she is still supporting students. Approaching the 7-year mark Flagship, Zoe revisits her initial goals of pursuing academic positions, and even roles in administration. Although content in her current role, she consistently looks at "places that are going to challenge" her and has not ruled out a "more formalized education component." Zoe is at ease with the status of her professional story and remains open to the next opportunity.

\section{Summary}

The intent to combine these individual narratives into a meta-narrative is the primary interest of this narrative inquiry, and is conducive to noting emergent themes that provide implication for research, practice, and theory. Still, it is pertinent to recognize how each woman's story encompasses details specific only to their journey. The benefit of delving deeper into their individual narratives is also finding the divergent points. As each woman explained the 
twists and turns, it was evident they make decisions based on aspects both personal and professional in nature. A broader look at the six pathways reveals key differences in how some women have benefitted from a supportive supervisor, while others have struggled to feel valued or empowered by the leadership at their institution.

In terms of encountering gendered situations where the majority are men, a couple of the women appear to have adopted strategies they believe are helpful in ensuring their voice is heard. Two women experienced job changes that were out of their control. While one woman comes from a family background where the pursuit of higher education is the norm, other women are the first in their family to obtain college degrees. A few of the women have advanced doctoral degrees, or are in the process of degree completion, stating this additional education is an important component to achieving their professional goals. Only one woman has established a clear professional development plan where she actively participates in intentional skill-building and job searches. Two women are unsure if student affairs are still their end goal, professionally.

Similarities across the women's narratives are numerous, resulting in the meta-narrative sought from this study's inception. Parallels include mentoring and experiences with supervisors, and their stories also reveal similar views on leadership, and the gendered components of leadership. In addition, the women appear to approach skill development using comparable sources and strategies. Each of these aspects will be expanded upon in the next chapter through an in-depth discussion of the themes, as well as an interpretation of themes through the theoretical framework. 


\section{Chapter 5: Findings}

This narrative inquiry focused on the careers of women midlevel student affairs practitioners, including what makes them successful and what role gender has played. Six women shared the narrative of their professional stories through interviews. The essence of these stories revealed that the women do much more on campus than their job title or position description reveals; yet, they displayed in the interviews a tendency to undersell their achievements on their resumes.

Through my review, I discovered that in addition to the responsibilities required by their job, they are involved in teaching; research; cross-campus collaboration; community service; as well as leadership at the local, regional, and national levels. In addition to these diverse experiences, these women are strongly committed to friends; partners; children; parents; siblings; as well as current and former colleagues, former supervisors, and employees. Those who completed the sketch activity provided more detail and depth to their experiences, which enabled me to see how they visually represented their decisions and their interests with colors, symbols, and illustrations. During our conversations, I easily noticed when their tone of voice or word choice changed when talking about an aspect of their story. Paying attention to these nuances revealed which experiences brought them excitement or frustration, and which made them proud. All the women admit that no workday is the same as the next, and that they often spend much of their times in meetings or sifting through e-mail messages. They jokingly described many days where nothing went as planned. But they all seemed content that no matter the tasks they must perform while at work, they feel accomplished or proud at the end of the day.

While data were gathered through participant resumes and information submitted through a pre-interview questionnaire, the actual interviews comprised the main data source, including 
transcripts of the first interviews and drawings obtained from the sketch activity. While each of the participant's stories was unique, common themes appeared across the women's narratives of their professional life stories. This chapter introduces findings (see Table 2) through seven general themes and 19 subthemes.

Table 1. Themes and subthemes

\begin{tabular}{|l|}
\begin{tabular}{l} 
Theme \\
Subthemes \\
1. Reluctance to take full credit for career success \\
\hline Unplanned \\
\hline Strategic \\
\hline 2. Defining success through feelings \\
\hline Validated \\
\hline Motivated \\
\hline Redefined \\
\hline 3. Misalignment between supervisor philosophy \& actions \\
\hline Office norms \\
\hline Impact on supervisory style \\
\hline 4. Importance of mentoring network \\
\hline Support from same gender \\
\hline Unconventional support \\
\hline Building a diverse network \\
\hline 5. Similar view of gender \\
\hline Culture dominated by men \\
\hline Representing self \\
\hline Interactions with other women \\
\hline Gender as a strength \\
\hline 6. Similar personal stories \\
\hline Personal attributes \\
\hline Approach to work/life balance \\
\hline Approach to professional development \\
\hline 7. Advice for the future of the field \\
\hline Administrative tasks \\
\hline Bigger Picture \\
\hline
\end{tabular} \\
\hline
\end{tabular}

Although each theme was created from commonalities found across the six participants' stories, differences in the narratives are also noted. The following section provides a comprehensive look at the individual themes and subthemes, including direct quotes and 
illustrations from the participants, as well as a brief discussion of the theme. Next, I interpret the findings through the theoretical framework for the study, the Kaleidoscope Career Model (KCM) (Mainiero \& Sullivan, 2005; 2006) and specifically, the KCM's three parameters of authenticity, balance, and challenge. Discussion on how the implications have the potential to influence the student affairs field is found in Chapter 6.

\section{Theme 1: Reluctance to Take Full Credit for Career Success}

Through the narration of their professional stories, all the women in this study described their careers as having developed naturally, but when asked to elaborate, they provided specific examples of ways in which they have been strategic. Quick to acknowledge individuals who have provided them opportunities to develop professionally, they expressed gratitude when talking about mentors, supervisors, and colleagues. They spoke of how the encouragement of others gave them the courage to try new things; however, the women seemed reluctant to take full ownership for their personal contributions. The two subthemes, unplanned and strategic, reflect the dichotomy noted as women described their career successes in the interview. While most of the women initially spoke of decisions, particularly early in the career, that were unplanned or unintentional, all the women were later able to provide concrete examples of times they have been strategic.

\section{Unplanned}

All first interviews began with the same prompt: tell me your professional story. Most women began their story with a caveat that some of their career steps were unplanned, almost as if they were providing a preview that certain turns in their story would require clarification by the participant. Liana recalled the uncertainty she felt when finishing her undergraduate degree, yet explained how things kept working in her favor: 
I just started thinking, what am I going to do when I graduate? [I] ended up connecting with a woman, talking with her about jobs, [and] she's like "oh, if you want to do that, you should get a Master's degree in higher education." So, I put like higher education as a question mark. I had no idea that that was something ... a legitimate thing and so I started applying. I really just applied to one or two programs. It all happened really fast; I did a campus visit [and] I got accepted the next week and then they offered me an assistantship. So, that was really, I mean, it was kismet, just, it just truly fell into place, actually a lot of this really is like that. I don't know that I'm a super active participant in this.

After sharing this story, Liana continued to walk me through the steps of transitioning to graduate school, what she learned through her program and assistantship, and how she entered the job search for her first position. In explaining each of these steps, she continued to reiterate the fact she had no overall plan, and instead, one positive break seemed to lead directly into another.

Similarly, Ann recounted the decisions that led up to the point of her return to Flagship for graduate school and her subsequent employment at the same institution. "How I got here was kind of not really planned ... there was not necessarily a plan of what I was going to do." Zoe admittedly "fell into student affairs" and when she was already working in a different area of an institution, "they cobbled together this graduate assistantship like, 'well, [Zoe] can do this."” Betty's undergraduate experience and the guidance of other people provided a solid connection to her graduate journey and eventual career choice:

I got into the profession just like so many people do in student affairs of being involved. My supervisor and mentor took me to lunch and she said, "have you ever thought about 
grad school?" And I didn't know at the time you really shouldn't go to graduate school without getting it paid for.

This conversation led Betty to explore the option of pursuing a graduate degree and accept the offer from her mentor. This individual has continued to serve as a source of support as Betty's career in student affairs has evolved.

For the participants who completed the sketch activity, it seemed to assist them in talking through the turns of their story as they continually referred to something they had depicted visually on the paper. Cathy used the sketch as an opportunity to show turns in her path (See Figure 1) that might not have been entirely clear without further explanation:

So, what I wanted to show was really that I've made some moves that make a lot of sense and I've made some moves that haven't made not a lot of sense on paper but have made sense for me.

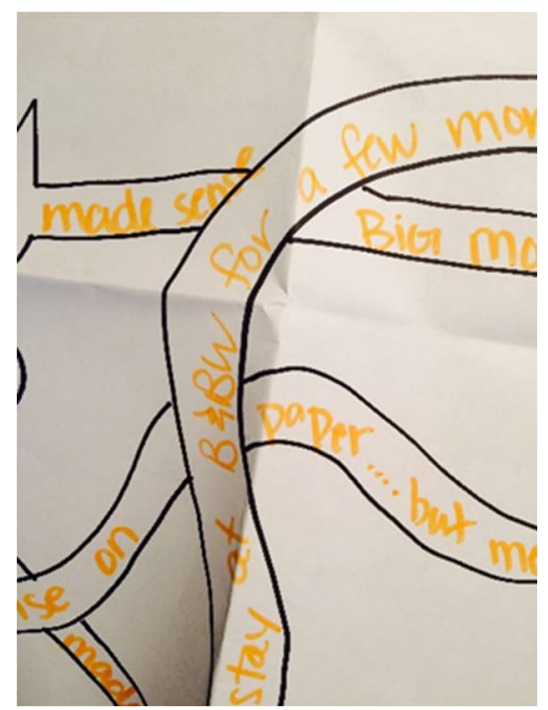

Figure 2. Unplanned career turns from Cathy's sketch

As Cathy continued to talk through her professional story up until the current time, she shared that she was unsure what her next steps would be. In her sketch, she depicted these feelings by using dashes and curves, ending with an arrow with a question mark inside (See Figure 2), but 
the uncertainty was not necessarily negative. In fact, Cathy seemed to feel optimistic as she described this section of her drawing, "this doesn't give me anxiety at all which is probably a little abnormal. Maybe it should give me a little bit."

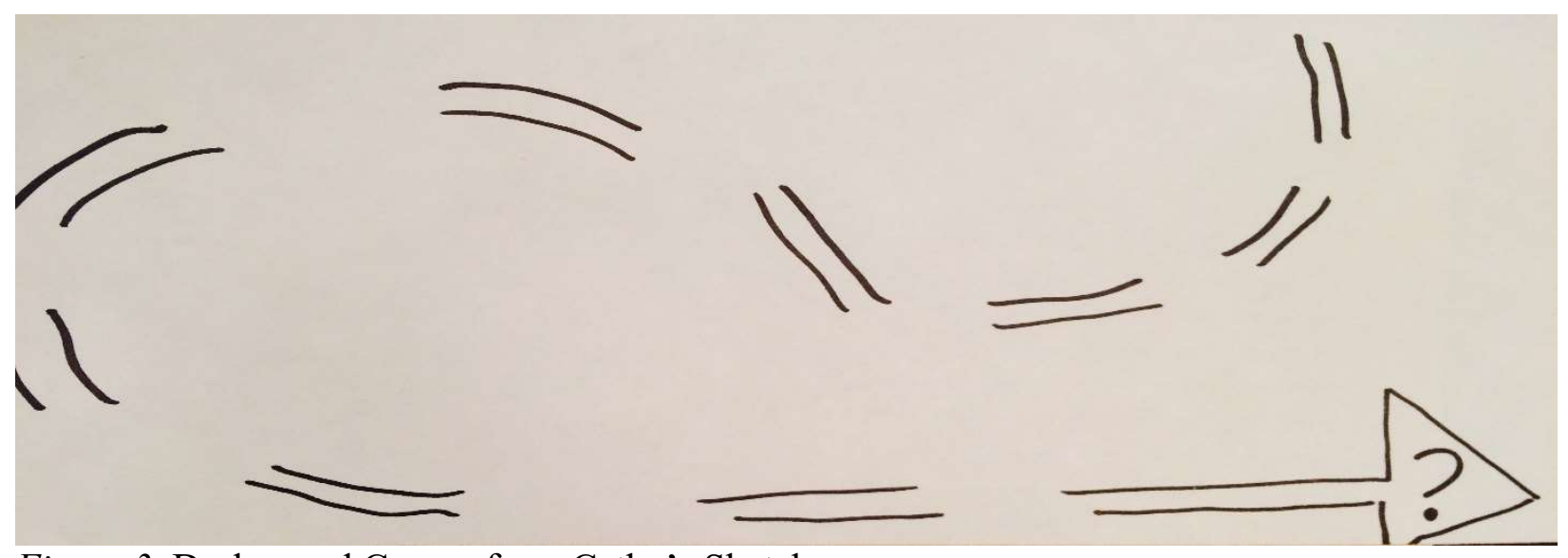

Figure 3. Dashes and Curves from Cathy's Sketch

Like the way Cathy depicted her career moves that may have been partly unintentional, other participants chose to use wavy lines or question marks to indicate turns in their own paths that were unplanned. In her sketch, Liana used a wavy line to depict a time in her career when aspect of her job stayed consistent and there were no significant changes in her career path (See Figure 3):

And then things kind of stayed like that for a while, just very, yeah ... it's the ebbs and flows of everything that happened until 2014. There's a lot that happened within there but not anything that really stands out one way or the other.

Figure 4. Wavy lines from Liana's sketch 
In contrast, Liana used solid lines to depict turns in her career that indicated predictable steps (See Figure 4), such as transitioning between graduate school and her first professional position.

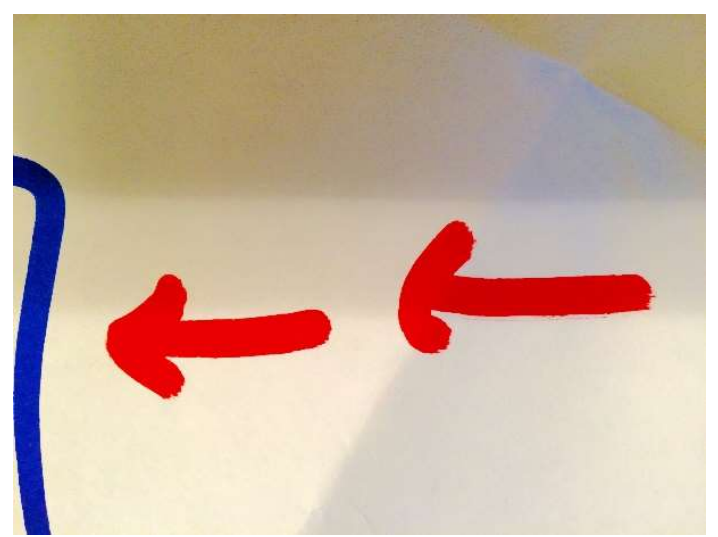

Figure 5. Solid lines from Liana's sketch

Continuing with the theme of depicting uncertain periods within career paths, Anastasia's story includes a brief time when she worked outside of higher education. Within her sketch, she drew a detour sign with opposing arrows to depict this turn (See Figure 5):

So, graduation, undergrad, Master's, and [I] had a slight detour. When I graduated, I couldn't find a job in student affairs and so I took a job in [a different field], which was awful, and I did that for 9 months and then, it was a grant-funded program, they lost their funding. But luckily, right as it was the last month I had to work, I got a job at [Midwest] and it was amazing. 


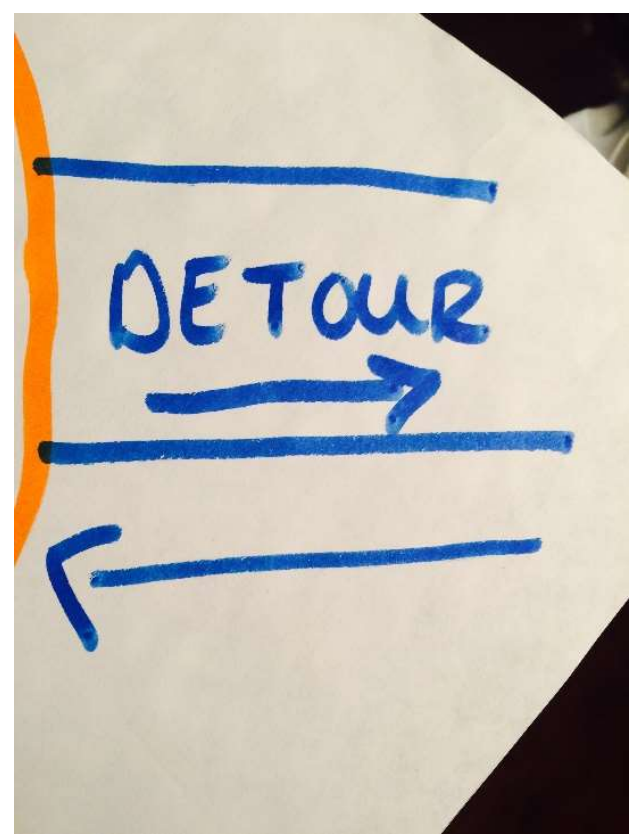

Figure 6. Detour from Anastasia's sketch

I appreciated Anastasia's explanation of this detour in her story, as well as how she referenced this time as uncertain, but that it clearly holds an important place in the success of her overall journey in the student affairs field.

After the arrival of her second child, Liana experienced an unplanned job change that was out of her control. In her sketch, she depicted this turn in her career path using capital letters to spell the word WHOA to indicate her feelings of being surprised (See Figure 6), "I came back from maternity leave and well and that, that was, it was, it was life changing." 


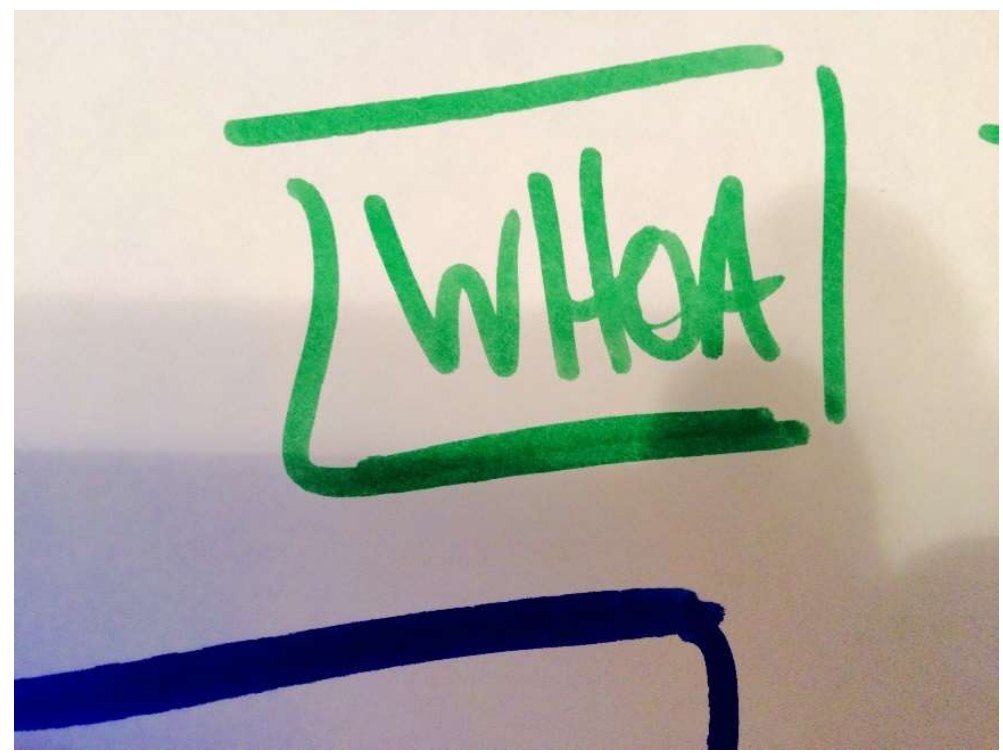

Figure 7. Whoa moment from Liana's sketch

Although these women began with the unplanned turns when explaining their

professional stories and walking me through their sketches, other examples they provided were most certainly strategic. By asking follow-up questions about specific jobs they had or interesting illustrations on their sketch, the conversation allowed the women to talk about decisions that were made intentionally.

\section{Strategic}

The women claim that their career paths have developed naturally, but when asked to expand further, they provided specific examples of how they have been strategic. Examples include taking initiative in choosing mentors, building relationships, and seeking advice from others (to be addressed in detail in theme four). The women have been strategic through their willingness to try new things and asking for additional responsibilities. Some shared specific examples related to education and professional development as efforts to improve themselves professionally. Cathy clearly stated:

I am definitely one that will ask for opportunities; where I see something that's not being done, I'll go like 'oh, I'll try that.' I tend to be more, it's just about 50/50 
introvert/extrovert, but professionally, I can make myself much more extroverted and sort of put myself out there and ask for those things.

Cathy attributes this type of courage in wanting to do more from the way she was raised, explaining that her family encouraged her to work hard and always do her best. She shared that her parents cared more about "effort than results" and this approach is something she has adopted now into adulthood, and within her professional life.

In Liana's interview, she shared that she has not "restricted [herself]," is "open to anything," and "was never told no." Zoe told me of a time she changed jobs and that she "just wanted to try something different and [I] ended up really growing and learning a lot." Ann is transparent with her supervisor in that she continues to review job opportunities, even though she is happy in her current job:

I'm not afraid to kind of test the waters and see where else I could fit in cause I think that's one of my biggest concerns as a professional is have I pigeonholed myself into ... a particular subset of a profession.

The purpose of this activity of applying for jobs is Ann's way of encouraging herself, but she also shares it as advice for other midlevel colleagues in student affairs, "get your materials together once a year, twice a year, throw it out there and apply."

A significant time in Betty's professional story is when she neared the completion of her doctoral degree. She made several strategic decisions regarding education due to its potential impact on her future opportunities, mostly related to work and family schedule adjustments so that she had time for writing:

So, it was a unique time but finally, when it got to be my last 6 to 9 months, I took a lot of time off [work] and just focused on my dissertation, 'cause at that point my husband 
said 'this dissertation is what is keeping us from the next step' and my career is our family's career. So, I knew that there was a lot riding so there was a lot of pressure to finish that dissertation. I had to take a lot of time to be able to make that happen and burn through a lot of vacation days.

Betty further explained that her family highly regards her career, thus the support they provided. An added benefit to taking the time off work for dissertation writing is that she could "be more focused" on her research and that she enjoyed the process more than she did when she felt pressured to put most of her focus on her job, thus placing the dissertation second.

The women have also been strategic in making career decisions that had little to do with their own professional journey and were focused more on their personal lives. While some turns in their professional stories have been arbitrary, many have been made with family in mind. Regarding the location of where Ann chose to pursue graduate studies, she shared that her partner was one influence in the decision. "My relationship at the time, my partner was here and he encouraged me to stay." Cathy also tied one of bigger moves to her partner at the time:

[I] moved with my partner, moved out there on a whim because we had both randomly visited [the location]. We both visited for the first time, thought it was a cool city, we're young enough to just think, "sure, let's just move across the country without jobs."

Once there, Cathy obtained a temporary job somewhat related to her undergraduate degree. Cathy later added that the next turn in her professional story was influenced by the same partner, but also by her extended family. "But then later that relationship ended, family got sick, so I needed to be back." 
Ann also shared about a time in her personal life that required her to reexamine where she was in her professional life, consider different options, and to be transparent about it all with her supervisor:

And you know, honestly, I had some things in my personal life that happened a few years ago that really challenged whether or not I wanted to stay in [college town]. And so, I had a conversation with my direct supervisor. I said "you need to understand that it has nothing to do with my job satisfaction, I love what I do, if I could take this and move it anywhere, I would ... but I may need to make some decisions for myself that no longer include me being here."

Through this conversation with her supervisor, Ann emphasized that the potential decision to move was solely based on the status of her personal life. During this point of the interview, I could sense this part of her story was difficult. However, Ann appeared ready to make sacrifices, including leaving a job she loved, to make the decision that was best for her at the time.

Several women mentioned supportive partners and that within their relationships, each person has taken turns putting their career first, particularly considering aspects like intentional career changes around starting a family, to provide better balance to family life, or to advance a career. In her sketch, Liana drew a stick figure family each time she added a child (See Figure 7), depicting the importance of this personal decision and its impact on her career journey. 


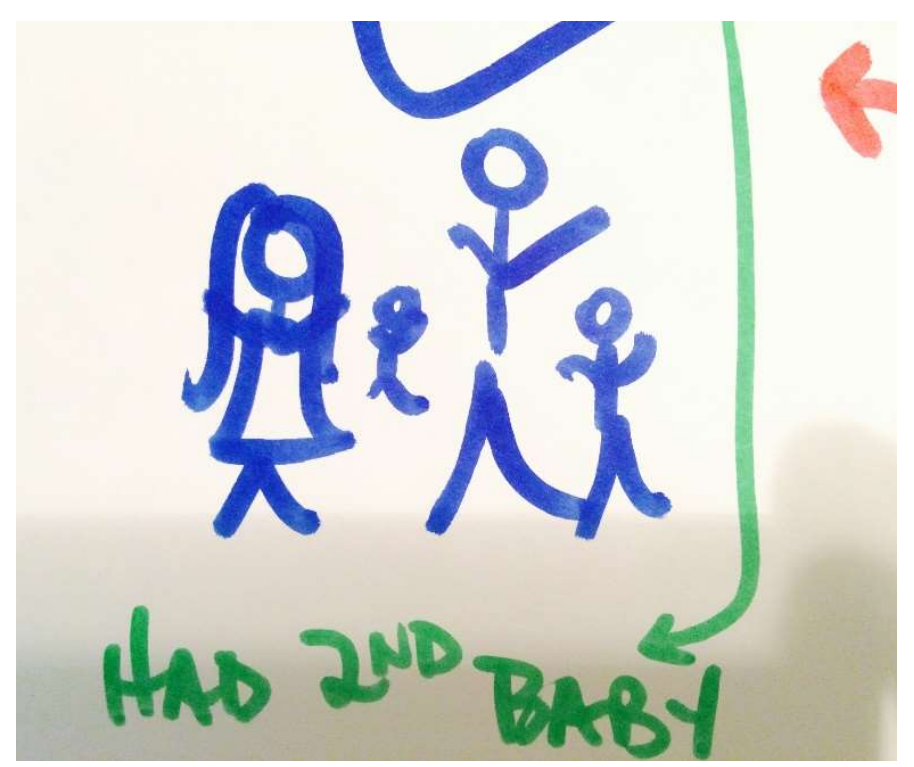

Figure 8. Family from Liana's sketch

As mentioned earlier, Liana recently experienced a job change that was unplanned, but resulted in her being able to make strategic decisions regarding her family. Her new role has positively impacted her work schedule and duties. One example is that she is no longer required to attend evening events on campus. Her duties are mostly contained to typical business hours, which allows her to accommodate her partner's work schedule, and that of their children. In addition, the change means she now feels as though she is less focused on her career and more focused on her partner's career. Since taking on the new role, she has spent time reflecting on her previous position, and the impact it had on her personally:

I kind of had that realization of like I need to ... this isn't just about my career, you know, this is about his too and to take that back seat a little bit and to be a champion for him. Looking back [at her previous position], I don't think I was happy as I thought I was. I said no to my personal life a lot.

Liana appeared relaxed as she described this opportunity for reflection, further accentuating how influential this recent time has been to her own professional growth. 
Anastasia's partner also works in student affairs. Although her partner is employed at another institution in a different city, he understands her professional responsibilities and the challenges that come along with her work. They have made many strategic decisions in their personal life to support Anastasia's doctoral work, and he has also provided critical support with family issues:

My mom [recently] passed away, but before that she'd been ill for a couple of years and doctor's appointments...we were trying to move her into a nursing facility. [My partner] was in the background doing [everything] because I couldn't be there. And so, I can be where I am because I had a human who was willing to do those things for me.

This is just one example that Anastasia provided to describe the vital role her partner plays in supporting her as a person and a professional.

Whether the turns in these women's careers were either unplanned or strategic, each turn comes with an explanation of how decisions were made, as well as their impact. Some turns seem to have occurred naturally based on situations and the people involved, while others require a bit more context for understanding. Either way, the language the women used in telling their stories is noteworthy, and is explored the following theme.

\section{Theme 2: Defining Success Through Feelings}

Also a consistent theme through all six stories, when asked to provide their own definition of success, the women used feelings to convey what is important to them. They believe they have been successful when they feel happy, proud, satisfied, accomplished, and if they have made an impact on others. Others mentioned actual tasks that make them feel successful like empowering others and creating access for students. Several indicated that 
defining success in such a personal way is a change from what they believed when they were entry-level professionals in the field.

From their stories, the following subthemes were identified - validated, motivated, and redefined. Collectively, it was clear the women have experienced situations with supervisors, colleagues, and students where comments or actions have validated their work, contributing to feelings of success. Similarly, other examples provided were times when situations occurred that motivated the women to continue their work, also contributing to feelings of success. Finally, since the women are midlevel professionals, they have worked long enough to have the ability to consider what success means to them, and for some, such reflection has caused them to redefine their personal definition of success.

\section{Validated}

Each woman provided examples of situations where she felt successful. Many of the examples included times they exercised their voice or accomplished a goal they had previously set. While some situations resulted in the women feeling immediate validation, other times the validation came later, once they had time for reflection. All examples provided within this context connected back to a desire to help others, thus the validation came from confirmation that others had benefitted from something the woman had done to improve a situation. Cathy explained that:

Success for me is an internal feeling, something that I can quantify as positive change. And so, that usually looks like, on the microlevel, positive change in a person's life, in a student's life, in a person that I have mentored or supervised. 
There were many times within our interviews that Cathy conveyed her desire to help others, and this sentiment shows up in the examples she provided when discussing turns in her story, tasks she enjoys the most, and relationships she has built.

Liana used a holistic approach to defining success for herself, which included both professional and personal aspect of her life:

I try to equate success to happiness more. So, being happy with my job, with my work, with who I'm with on a daily basis. Everything's [not] $100 \%$ all the time but I'm happy with my own personal journey.

Liana presents herself as quite comfortable with her current role and responsibilities, and even more so with her work environment. Supplementing the language used, her tone and non-verbal cues are also key indicators of how specific emotions are tied to the different aspects of her professional journey.

Some examples of success shared by the women were situated at the end of the workday when they had time to reflect on what had transpired since the morning. Ann has time to think about the day's events as she is walking back to the parking garage. "Most days I still feel accomplished because I got through things that still had an impact on others." Similarly, Betty takes success one day at a time:

Feeling comfortable with the job I did, excited about the day tomorrow, content in where my family is, is my house where I want it to be, are my relationships where I want [them] to be. So, I look at success as a little bit everyday, not necessarily title. Betty's point underscores that feelings of validation are personal. These end of day reflections she and others spoke of do not include others, and are focused solely on how the individual woman feels about the work she has done. 
Anastasia acknowledges the challenges in defining success as a student affairs professional in that the work is multi-dimensional, comes with numerous tasks, and can be emotionally draining. "Defining success is really hard for me. I sort of feel like I have to be a superwoman, and so [I'm] maintaining." In her sketch activity, Anastasia even drew the superman symbol to represent her current role.

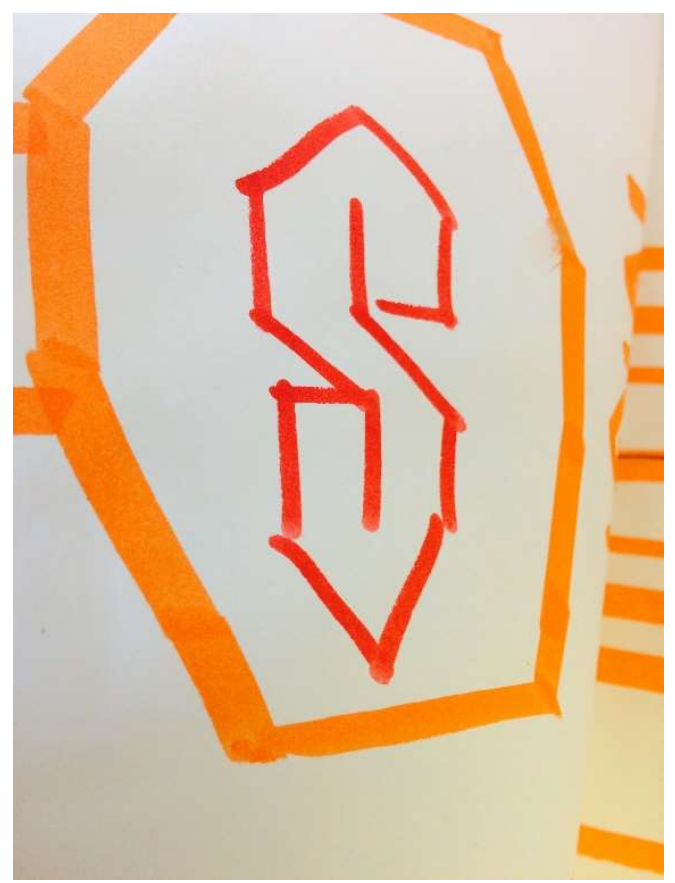

Figure 9. Superwoman symbol from Anastasia's sketch

Even though Anastasia feels the weight of her many responsibilities, she often channels advice from a mentor in the field, who told her "just do your work well and people will notice." This piece of advice seemed to assist her ability to consider her hard work as validation to remain in the field, and equally motivated her to collaborate with colleagues.

\section{Motivated}

Many of the women feel encouraged when they can motivate others, which then produces feelings of success. Anastasia recognizes the influence she has on others, including her students and staff. "I'm a role model, whether or not I want to be. But I have an obligation; it's one that I 
gladly accept to pay back forward." Betty has also noticed her part in empowering others, specifically the professional staff members who directly report to her. "Being able to develop staff and to see them grow and to see I learned from them as much as they learned from me, that's invigorating and to see us have this reciprocal relationship that's very engaging to me." Zoe finds similar motivation when she inspires students to think critically and grow as people. A particular scenario that occurred recently included Zoe "being able to see students stop and think. The whole concept is [both] common sense and critical thought, [thus] working with them in different ways and seeing that potential grow and feeling like there's some hope."

Certain instances that occur within a workday seem to perpetuate these women's desire to remain in the profession and continue the work they are doing. Betty remarked that:

Every day I usually come very excited or I find there's something I'm excited about to do or a new endeavor; the joy that I find is in doing something new. I love to work on different projects; I'm an idea person.

This is not the only time Betty refers to responsibilities, projects, and interactions with others as sources of motivation. Her story includes several examples of collaboration and teamwork as springboards to feeling inspired about the profession, as well as instances where navigating challenging situations also resulted in feelings of motivation.

Cathy feels a similar sense of pride when reflecting on success and admits that her age and stage of life give her "permission" to continue doing what she loves. "It's neat to be able to introduce yourself and talk about what you're doing and feel like you're able to explain that you're positively contributing something through the work that you do; it feels very empowering to me." Anastasia explains that feeling successful is tied to the realization that the work she does 
is never completed. "I think, is [this] successful? ... it doesn't [always] feel successful because I feel like we could always be doing more; there's a lot of work to be done."

\section{Redefined}

Most of the women admit their current definition of success looks much different than it did earlier in their careers. At that time, they may have defined success by status, title, pay, honors, recognition, and which individuals were in their network. Using a bit of humor in providing her definition of a successful student affairs career, Zoe quipped, "It's definitely not the money, it's not the rewards." She added:

My version of success 10 years ago, [was] “I'm making money, I have a 401K, working and doing my master's and I'm going to start a doctoral program." I had these goals, I reached them, and so for someone who was way more type A, [it was] good for me to figure out what do I want to do next and being okay with it.

Underscoring that definitions of success are personal; Zoe's example shows that redefining success is also based on an individual's lived experience.

Agreeing with how one's perspective on career success can change with time and maturity, Betty reflected on specific aspects typically associated with success:

Earlier in my career, I used to look at where am I at, what's my title, what's my salary, what school am I at, who's asking me to present at different things, do I get to be able to be up for awards? I started to think, if that's how I define success, I might really be miserable. If I don't attain those, what does that mean? So, I redefine success in a different way for me. If those things happen, that's great, it's icing on the cake. 
Betty's explanation of how she has redefined success for herself strongly mirrors the example provided by Zoe. Both accounts convey a sense of maturity that aligns with the fact these women are midlevel, thus have progressed further into their careers.

The fact all women in this study chose to describe their definitions of success using feeling words indicates they may have reached a point in their career where their own view of success outweighs the opinions of others. Perhaps they have identified individuals they deem successful and paid attention to how they display feelings of success. Connected to the next theme, these women appear to have a unique ability to observe people and situations around them, taking cues of what might work [or not work] for them personally.

\section{Theme 3: Misalignment Between Supervisor Philosophy and Actions}

All six women noted varying degrees of misalignment between what a current or former supervisor stated as their philosophy and the actions they displayed. This was particularly predominant with philosophies associated with work/life separation, professional/personal life boundaries, and professionalism (or lack thereof). From their stories, the following subthemes emerged: office norms and impact on supervisory style. Several women talked about the influence of the office norms at their place of work, and particularly the ways in which their supervisors either establish and uphold the norms, or alternatively, the ways the fail to exhibit stated philosophies. Such noted contradictions may result in employees who are confused about expectations for their own behavior. Furthermore, some of the women see a direct connection between how these experiences have impacted their own supervisory style.

\section{Office norms}

Also referred to as work culture or office culture, the term office norms used here indicates common practices found amount staff members who work in the same setting. Less 
formal than policies and procedures that may be found in all offices, there are norms that staff are expected to understand and comply with, even if they are not outwardly stated. Norms exist between colleagues, as well as between supervisors and staff. Specifically, the following examples portray instances where supervisors insinuate one thing but display opposing actions.

An experience between Betty and her supervisor occurred when she was working diligently to meet dissertation deadlines to complete her doctoral degree:

My supervisor kept saying "I support you, I absolutely support you" and I think he meant it, but the problem was his actions weren't. [He sent] e-mails like "why aren't you following up on this? Why isn't this happening? We need to move in this direction" and more.

This disconnect between stated support for her to complete the dissertation and pressure to complete daily tasks resulted in Betty feeling conflicted. She later referenced this time in her story by sharing, "I got more support and encouragement from the people that report to me than higher up." This notion of receiving support from others is explored within the realm of mentoring in Theme 4 .

Recalling an incident earlier in her career, Betty was presented with an opportunity to help expand her knowledge in student affairs by working part-time in another office, but was met with resistance from her supervisor at the time. Although they had a positive working relationship, he was not entirely supportive of her working with another department and director for one semester. When Betty presented the option, "my boss said, 'you can do it [but] you have to do both jobs.' Instead of looking out for my welfare, he was more worried about his welfare."

Betty was just starting to understand the culture of student affairs, which from the start, has been inherently supportive, collaborative, and invested in staff, especially when individuals 
seek to build their skillset. The reluctance displayed by Betty's supervisor to allow her to assist another office went against the culture she believed to understand. However, due to her sincere interest in growing as a professional, she made a personal decision to work both jobs, even though it meant working extremely late nights and on weekends.

Anastasia's example of misalignment by a supervisor focuses on what can happen when a leader stops leading. Additionally, their behavior in the office is drastically different from what is seen by the rest of campus. The following story is from a time when Anastasia was hired in a newly created position within an office that would allow her to get back to the work that meant so much to her. After dedicating 5 years to the office, the work environment turned into a stressful situation due to a supervisor who allowed his own personal struggles to impact office culture. The experience had a deeply personal effect on her at the time:

I had an awful supervisor who was going through his own stuff and, instead of dealing with it, turned it on the staff. And so, everybody who's in the office left. That was over a 2-year period. Everyone left, except for me and one other person. It got so bad that he convinced me I shouldn't work in student affairs; [he] convinced me that I was awful at my job. It was very bad. My self-esteem took a dive and built some like paranoia and like people are talking about me, about my job.

During this time, Anastasia searching for jobs outside of higher education, but is now thankful none of them worked out. She found support from her partner and campus colleagues who knew her well, and from colleagues from other institutions who were involved in her same professional association. Finally, the problematic supervisor was eventually replaced by an interim director who Anastasia describes as "so incredibly developmental." While this new supervisor was only in the position for a year and a half, his impact on Anastasia was profound. 
“[He] really built me back up; I think if it wasn't for him, I probably still would have left student affairs." Anastasia's story showcases the degree of impact that negative situations can have on an individual, hence the challenges that come with misalignment in office norms.

Liana's supervisor for several years in her first professional position in student affairs was a woman, about whom she noted:

A lot of boundaries were blurred. Hindsight [being] 20/20, that getting a text message at 6:00 in the morning that was not an emergency, was a regular occurrence, even though there would be conversations like "oh, when you're home, you're home or like, you know, this is work or it's time to go home from work or do home." But so, [her] actions and philosophies didn't line up.

Liana attributes the blurred lines to the fact she and her former supervisor had developed a friendship, and that she was a new professional. She also shared that although she may have noticed the discrepancies in her supervisor's philosophy and actions during the time they worked together, her full acknowledgment of their impact did not come until Liana had transitioned into a different office, with a new supervisor. She describes the culture of her new environment more positively, including job tasks that can be performed mainly during business hours, allowing Liana to be fully present with her family when she is not at work. She also benefits from a supervisor who appears to understand her personal commitments. While it may have been small differences Liana noted at first, these new experiences revealed the unhealthy work environment of her previous position.

Specific challenges experienced by these women seem to revolve around expectations for communication outside the regular workday hours. Ann described her current supervisor (a woman) sending e-mails on the weekends in this way, "she often does a brain dump, but does not 
expect a response." When asked for clarification, Ann explained a caveat to receiving messages on the weekends; her supervisor has conveyed that if she e-mails on the weekends, she does not expect an answer. We then discussed the difficulty in remembering this caveat when attempting to enjoy personal time but see e-mails arriving to the inbox.

Cathy is in a similar situation with a woman supervisor, and is trying to achieve a healthy balance for herself in terms of protecting personal time within an environment where not everyone feels the same way. "The expectation and the culture is definitely that you're on it all the time." Cathy described her current supervisor in this way:

She is a nighttime e-mailer. She goes home, has family time, and then she has, from what I can tell, probably another 45 minutes to an hour that she works on e-mail from home every night. But her expectation has never been that I respond right away. But that's been difficult to navigate because you feel like if your boss is doing it, is it really politically okay that I'm not?

Cathy shared an example of a time when she arrived to work at 9:00 a.m. and began her day as usual, in her office. Only later did she learn that most of her colleagues were attending an "emergency 8:00 meeting." Apparently, those individuals had "checked their e-mail the night before and all knew, and then, there I was." Although Cathy quickly learned the information she missed at the meeting, and can look back on that story and laugh now, we discussed how this culture has evolved. We pondered what campus culture was like prior to e-mail becoming such an integral communication tool, specifically with regard to her story about the missed meeting. Zoe's current supervisor also communicates at nights and on weekends. "[She] sends emails but doesn't expect a response." Though, Zoe discloses that she may have interpreted this behavior differently when she was a new professional. "Advice [I was given] back then was to 
watch this person and emulate them." Perhaps now that Zoe is a midlevel professional she has the insight not to respond to e-mails on the weekend to protect her personal time, and is unlikely to adopt such behaviors when supervising her own staff.

Betty shared her observations of an administrator who was a woman. While this individual described a culture of supporting women, Betty did not feel the philosophy was always enacted:

Having a Vice Chancellor being a woman saying we have to support women but I don't really see it in her actions. So, for years, she would say "we have to help women, I'm at the table with men, I've done this a lot," but there's not actions there to be able to see that support, so to speak. It's not vindictive or vicious, it's just that's not where their [some woman administrators] interest is and that's not where their thought is. They mean well but it's not there.

Although I did not ask Betty to expand on specific ways the administrator could have carried out her philosophy of helping women, she did mention making connections with people. "You have to be really present and be connected with folks" and this individual was not always intentional in connecting to other women in the organization. All the examples shared by the participants about office norms placed the women as observers of behavior and receivers of information, serving as influences on their own practices.

The above stories included examples of supervisors who are men and women. Specific to the stories about work/life separation, the fact the women participants chose to provide such examples only for women supervisors is noteworthy. While some women may desire women leaders who serve as positive examples of balancing their personal and professional lives, these stories are of women supervisors who do not. 


\section{Impact on Supervisory Style}

The women acknowledge these negative examples of supervisors whose actions did not support their stated philosophies have influenced the women's own supervisory style. Where unhealthy habits were noted, the women were intentional not to replicate with their own staff. Cathy shared:

I have really only had one not great supervisor and I learned just as much from her, if not more. [She was] one of those people that would tell us to do things but wouldn't do it themselves and wouldn't put forth the effort themselves and I said, "I will never do that."

Cathy then offered examples from her own career where she has been sure to lead by example. In one job, she took out the trash. Within her current role, she oftentimes staffs an information/resource table, even though tabling is not necessarily her favorite task.

The unhealthy habit displayed by Zoe's current supervisor has caused her to seriously consider the negative modeling she sees from women administrators in her department:

I look at her and I look at some of the other women in our department, seeing their lives. I'm like, I don't think people realize, I've not had an individual meeting [with my supervisor] without her falling asleep. But, how do you navigate that, knowing it does not have to be this way? From the outside looking in, no place [is] worth my health, my soul, you know?

Although the negative practice Zoe observes in her workplace are consistent in their occurrences and quite prevalent, she does not believe the environment is conducive to discussing such issues.

This section highlights the influence of supervisory style and practices on their staff, as well as the work culture our leaders uphold. When staff notice misalignments between what a leader says and what they actually do, there arises potential for mistrust and a decrease in morale. 
Long-term effects of such an environment include lack of commitment and buy-in to the institution or the field, as well as how the staff will someday supervise others. Gender aside, the misalignments noted within this section could easily be about men or women supervisors. However, the issue of women supervisors who are unsupportive of a woman employee's need for work/life separation will be explored further in Chapter 6 .

\section{Theme 4: Importance of a Mentoring Network}

Numerous comments by the women in this study support the value of having more than one mentor. Intentionally building a mentoring network may include peers, employees, individual of the same race, and colleagues outside of department or outside of field. From the stories shared by women who have benefitted from mentorship, three subthemes were created: same gender support, unconventional support, and building a diverse network. Many of the women shared examples of same gender support displayed by women who gave them opportunities through their career. Other stories told of unconventional support, or times when support came from unexpected or interesting sources. Related to the previous description of unlikely mentors, most of the women talk about their intentions in building a diverse network.

\section{Support from Same Gender}

Even though two participants provided examples of men who have served in a mentoring role at some point in their careers, five participants identified a specific woman mentor, or multiple, at some point of their student affairs story. For both Ann and Betty, it was a woman they had worked for during some portion of their undergraduate experience. Ann was approached by her former supervisor, "She's like, 'so I've got an interesting idea for you, how would you like to take grad school a little slower but work for us?' At that point, I was still weighing my options and the stars were aligning." Although the offer was not something Ann 
initially considered a possibility, the fact this woman believed in her potential to handle work and school motivated her to say yes. Significantly, this individual has continued to serve as a source of support to Ann, and remains her supervisor today.

For Betty, it was a similar conversation with her former supervisor that started informally, but resulted in an opportunity worth serious consideration. "She took me to lunch and she said, 'have you ever thought about graduate school?' I said 'well no, not really.' She's like "well, think about graduate school; I would pay for it." Betty reflected that until that conversation, she was unaware that most students pursuing a graduate degree in the student affairs field do so with an assistantship that has an attractive financial component. Therefore, the offer made by this woman over lunch was more comprehensive than she initially realized.

Anastasia benefitted from a team approach that supported her through her undergraduate journey, and like Ann, these women mentors have remained supportive of her as her career evolved, including the current time:

I had two amazing women in my life who were my mentors, and was like 'I want to be like them.' One of my proudest college moments is walking across the stage at graduation and [they] were there to give me a hug.

Anastasia credits these women in serving as an integral part of her undergraduate experience, and throughout our first interview, she made several references to the impact their work has on her passion for helping students.

Also benefitting from consistency in mentorship, Cathy sums up her experiences with mentors in this way:

I've had someone like that [a mentor] in my life, it's been a different person, but I've always had a mentor. Typically, a woman who is at least a few years older than me, 
whose advice I look to and whose opinion I value. They aren't people who would ever tell me what to do. They are always people who would ask me questions, guide me in that way to get there for myself rather than saying, 'well I think you should do this.' They have mostly been women, almost primarily women, or a non-binary person who identified more on the spectrum.

Cathy referenced some of these individuals in various parts of our first interview, particularly in how the connections were initially made. Her personal approach to mentoring is explained later in this section. In addition to having women mentors early in their educational journeys, the women in this study continue to benefit from positive examples of women colleagues now that they are working in the profession. The support has come at times of success and times of challenge. Liana shares that "these women have been really impactful through different times, saying, 'this is doable."' A specific situation that tested Liana in her current job resulted in support from an unexpected place:

Another female administrator who didn't give a ton of advice but just said "Are you okay? You know, you're smarter than this,' and just that little bit of support, and from someone that I never would have really expected it from, not a supervisor, not even anyone in my division ... that was important.

This story from Liana came at a time in her career when she had recently experienced the unexpected job change referenced earlier in this chapter. As she navigated a completely new role and relationships with campus colleagues, this support from another woman was meaningful, and connects to the next subtheme. 


\section{Unconventional Support}

The women shared stories of support that are not always top-down hierarchal mentorship. In fact, some examples point to support from colleagues and peers who may be at the same career stage or life stage, and may become colleagues to grow with. Ann is proud of the support she receives from close women colleagues in her department, "I have many women around me to watch grow as professionals, and grow with them as professionals." She describes her department as "atypical" in that the leadership is predominately women, and is aware that the rest of campus looks quite different.

Like Ann, Cathy embraces the importance of colleagues who work well in supporting one another, as evident many times in her professional story:

But it was really about the people, and I think that's consistent through my story. I could work as a trash collector if I had a great team. I think that you spend often more time with your coworkers than you get to spend with loved ones, right?

This perspective of individuals working together as a team is important, given its connection to comments made by other participants. For example, an experience from Betty's career revealed that mentorship may also come from professional staff members supporting their supervisors. When Betty was finishing her dissertation, and taking days off:

It was people that I supervised that rallied around me. I actually found later on e-mails that I didn't get that they would send to each other saying, 'look, she's got this thing due in a week, she's at the library, if you need anything, let's work on [it with] each other, let's not try to e-mail her.' And I didn't know they were sending [these] things. In watching Betty tell this part of her story, it was evident that these people mean a lot to her. She later emphasized, "I get more support and encouragement from the people that report to me 
than higher up." Even though this support from her colleagues and staff was somewhat surprising, other women have taken intentional steps to identify multiple mentors.

\section{Building a Diverse Network}

Four women have been strategic in choosing mentors who can relate to similar experiences or who can provide different types of support when different support is needed. Anastasia has:

...sought different types of mentors. So, I have the person who's been in the field a long time. I'm like, "tell me what to do." I have female mentors where I'm like, "this is a boy's club "cause when I go to those tables, I might be the only woman, how do we show up?" And I have women of color mentors 'cause, it's all so different, like how we show up, how I'm allowed to show up, and how I'm perceived on campus.

This type of support can be especially helpful when challenges present themselves and then one needs to look outside the traditional mentors. As she struggled through the experience of the challenging supervisor, Anastasia found guidance from outside of her institution. Her desire to do student affairs work was:

Reinforced by colleagues who said, "You're pretty awesome. We like you. We want you to do things for [the professional association]." It felt like such a tight bubble at [the institution] and to be able to connect with other professionals in the field; this is bigger than this bubble that I'm living in and I could go other places and do this work and be well received.

Anastasia continued developing relationships with these individuals, thus increasing her involvement in the professional association. For many years, serving in various roles was a way to connect to individuals doing similar work in other spaces, affirming her connection to the 
field. Although she has since transitioned to other professional development opportunities, the relationships made through her first one are meaningful today.

Regarding the intentional steps these women have taken to build a mentoring network, Cathy has developed a unique approach that is more personal and focuses on connecting with people individually. The practice includes reaching out to someone and asking, "can we go out to coffee some time?" She describes the process as "super scary to do" but gives the history behind the tactic:

When I was a grad student, I was sitting next to someone at a workshop and they were talking about finding mentors. My stomach hurt and I was nervous but I turned [to an individual] and I said "Can we go out to coffee some time and would you be my mentor?" And it was super awkward and awful, but we are good friends now, and I continue to look for her for advice on different things. It doesn't always work out that way, but usually, people love to talk about themselves. People love to mentor and give back in this field.

More than an informational interview, Cathy's approach is personal. She shared that after having success the first time she used premise of going to coffee to get to know someone better, she was encouraged to continue the practice. Even now, when she identifies a person she wants to know better, or believes they have significant experiences to share, she invites them to coffee. Not all become mentors, but they all add something to her professional story.

Mentors may have different roles. Some may possess an ability to encourage while others are more skilled in challenging or pushing individuals to persevere. Anastasia described the various roles in this way. "You have to have like different types of people in your life ... the straight shooters and the warm, fuzzy people and the 'it's okay, you can do anything' people."” 
The mentorship these women have received from others has impacted how the approach the task of supporting others. Cathy embraces the task. "I have been mentored by just the most amazing people and I want to be able to be that for other people and sort of pay that forward, for lack of a better phrase." However, she recognizes that the although her most influential mentors have been women, the concept of mentorship should not be gendered:

I don't expect that all women need to be nurturing and that all women need to be mentoring. That's not an expectation. But, I think it is something cool that is often associated with the gender that I identify with, and so something that I embrace. [It] doesn't have to be that expectation for every woman.

The possibility of mentoring being viewed as gendered connects to several other points discussed in this study, including student affairs as a helping field (including the language around competencies), formal networking opportunities in the field created specifically for women, and the KCM framework applied to women's careers. These topics will be discussed in Chapter 6 . Contrary to deliberate methods of finding a sole person to serve as a mentor, Betty finds support from a variety of people and places. “I really didn’t have a strong mentor so I just kind of picked up my experiences or my inspiration from different places. I never had one person.” Similarly, Zoe has never had a formal mentor; "I've never even asked someone." But Zoe did share examples of key women in her network who are instrumental in serving as role models or helping talk through situations, her "networker friends; I do have a relatively strong support network." Listening to all the women share about sources of support, it was apparent that the value added by mentors to the women's' lives and professional stories is more important than how they were identified. 
While each woman approaches the concept of mentorship in different ways, their stories stress the importance of support as they progressed through their professional journeys. Sometimes the support comes from expected places, often other women who can relate on topics specific to women professionals. Other support comes from surprising places, or from people one might not have expected. Finally, whether mentors happen to emerge naturally or if women design a strategic plan to build a network of support, the positive impact of mentorship among their stories is unquestionable. Related to the above stories of women serving as mentors for other women highlights the topic of gender. The next theme explores how these women talk about gender within the workplace, and over the course of their careers.

\section{Theme 5: Similar View of Gender}

Overall, the women mentioned gender bias as more of a feeling they have versus providing examples of blatant discrimination. This is especially relevant to the fact these women perceive the student affairs field to be led mostly by men. Within this section, three subthemes emerged. First, culture dominated by men frames the stories the women told about their professional interactions with men. Next, representing self describes the awareness of gender that these women have, and how the make intentional choices on their responses within gendered environments. Just as their relations with men are important, their interactions with other women are worth examination. Lastly, gender as a strength is a commonality among their stories.

\section{Culture Dominated by Men}

All the women in this study refer to higher education and student affairs as organizations led predominately by men. Although this perception certainly varies between departments, functional units, and institutions, it remains an important factor in this section since the view is shared by all six. 
Ann's current role places her in frequent meetings and with the men in her department. Speaking of this type of interaction, Ann shares:

It's more of a feeling. I don't know that I've ever directly experienced any negative comments of like "oh, it's because she's a woman." It's a general feeling. I feel it more from when I've sat in a room, and questions seemed to go to the men and not the women. It's like "no, we're the ones in charge of the project," so, yeah.

While this willingness to ensure her voice is heard largely connects back to Ann's confident personality and emphasis on professionalism, she also benefits from unique leadership. Ann communicates that her supervisor is a woman who has personally made significant strides within in area of student affairs that has long been dominated by men. Thus, her supervisor has created a work culture where gender stereotypes are challenged.

Examining the leadership in her current department, Zoe especially notes behaviors exhibited by the men towards her, and reflects how the different aspects of her identity may impact how she is treated. She also noted the race and gender of the department's director and how his identity may impact how he is treated. She asks herself, "Am I perceived differently? The support [the director] gets, for a variety of reasons but most particularly [because] he's a White male, would a woman get that? Would a woman of color get that?" Throughout her story, Zoe has examples of ways she has been treated based on different aspects of her identity. She is not alone.

Anastasia connects her own experiences as a woman in student affairs to the larger field, and questions the dynamics. "Student affairs is very female dominated but the power's still given to men. How do we navigate that and how do we show up in these places where we're not supposed to be?" Betty noted the formal ways in which men are privileged in the student affairs 
profession, and ways she has been treated differently because of her role as a mother. "I've seen the difference in pay, I've seen the difference in 'well, you're a mom and so you have to go do that' but I'm also a professional." How these observations of culture might be received by leadership at the institutions where student affairs professionals work, as well as to the larger field of student affairs, warrants further dialogue. Of similar interest might be how individual women operate within gendered cultures.

\section{Representing Self}

The women are transparent in that they possess the ability to choose how to respond in situations and to certain people, but that their choices are not always easy to make. According to Zoe, "I think it is the minute I walk in the door how I choose to present myself." She recalls more "telling moments" that occurred recently, one in which a task she had overseen for a couple of years was suddenly given to a man in her department, although in the past, Zoe was the only staff member willing to volunteer. The task entailed Zoe serve as a liaison to new administrators on campus. For 2 consecutive years, the new administrators were women and Zoe performed the task well. With the most recent new administrator a man, an awkward discussion at a meeting resulted in a quick decision by the department director to re-assign the liaison role to the only man in the room. Zoe describes the decision as "fascinating."

Regarding interactions with others, Liana is also cognizant of gender but carefully decides if she will address concerns:

One day I had a [man] who gave me a talking to about our decisions and still did not communicate with me at all after that, but apologized to my supervisor [a man] for how he reacted to me. Never once did he even send a note to me to apologize for it. I know he wouldn't have talked to a man in that way. So, how do I not let someone talk to me a 
certain way just because I'm a woman? Because it's real; it's easy for them to do that. But also not pick a fight about it.

The language used by Liana in this example is interesting, particularly her assumption that addressing the behavior would be considered as picking a fight with the individual who had shown her disrespect. This uneasiness associated with determining reaction and follow-up action is shared by Anastasia and Betty.

Anastasia struggles in choosing when and how to exercise her voice, and connects this struggle to the ways in which women are socialized into the profession:

Learning as a woman to assert myself ... I spend a lot of time understanding that. In spaces where there are a lot of men, I'm never the first person to speak up because I'm a woman so we're not groomed to do that. I'm a person of color so we're not groomed to do that. Recognizing, understanding why I might not use my voice in a space and then changing that behavior. So, forcing myself to speak up in meetings.

Anastasia's trepidation in navigating gender (and race) dynamics within her work culture influences her understanding of herself. She expresses it in this way:

I have a strong commitment to my values, and understanding how they align with who I am and how I show up. And the places where that differs from what the institution is asking of me. Being able to find a happy medium between the two.

This awareness of self can connect to the way women frame their actions and behavior, and how they justify these decisions.

Several of the women mentioned times they have made the decision to act less like a stereotypical woman in terms of language and behavior to fit in better with an environment 
dominated by men. However, Betty's example is the most remarkable. Her career is full of examples where she was (and is currently) one of the few women in a meeting space of men:

For me, I definitely hit a wall of being accepted in the old boys' club with what I do. And, with our alumni who are men. I have learned how to have to speak boys, is what I say, and how to just kind of listen and almost tone down some of my female persona, and my thoughts and views, and just trying to listen and be at the table with them. The more I am, the more they open up, and the more I see really where they're at and who they are. So, it's allowed me ... I don't agree with it, but by me being able to listen, I know where they're coming from.

This story from Betty encompasses more than adjustments to behavior based on gender, but also expectations, language, and gender performance.

\section{Interactions with Other Women}

Stories of how gender intersects with the professional story were not all about men, with some mean girl examples. Recounting the early days of a new job Betty took years ago, "there were some mean girls in the group, so there was some of my colleagues who weren't really welcoming to me." She navigated this by "not analyzing it too much" and getting to know the colleagues who were welcoming so that she could sustain her excitement for the new position.

Zoe vividly remembers an unexpected interaction with a woman faculty member of color. As a prospective student visiting a new campus, Zoe met with the professor who would be her potential advisor. The meeting occurred in a private office: "I remember her touching my hair and saying, 'I like my color but I wish I had your hair; my life would be easier.' That was my first meeting. I'm like ...” Though Zoe shared there are multiple stories where comments were made about her hair, this particular interaction clearly influenced Zoe because the comments 
come from an academic with whom she could be working closely (for reference, Zoe did not end up being advised by this woman).

Liana's perspective on women working together is that careful steps must be taken to establish a positive and supporting environment:

Sometimes it's hard for a woman to trust another woman, and it's not an overnight thing, like it takes time. A little setback can make a big impact. The biggest thing I've learned about having female colleagues is that they can be amazing colleagues and that it doesn't have to be a friendship.

Liana applied this perspective in comparing her current work environment (the newer job) to that of her former office. She noted the main differences came in the form of trust (that everyone is doing their job) and communication as a strength among colleagues, and between supervisors and staff.

Compared to the previous subtheme, similarities exist in how the women respond to challenging interactions with men and women in the workplace where gender is a factor. Pathways to explore this further could include assessments of language and behavior around how women react to gendered treatment by both women and men colleagues, as well as the outcomes of such interactions.

\section{Gender as a Strength}

While the interview conversations about gender first revealed challenges the women have encountered, several women also included examples of how they have used gender as a strength. Created as a separate subtheme, examples of gender as a strength permeate all other subthemes and stories within this section. However, three specific stories are highlighted here to illustrate the prominence of this concept. 
Anastasia encourages other women to take ownership of the role women play within gendered organizations, environments, and within the larger field of higher education:

Stop apologizing, use your voice assertively. It may not feel comfortable but what you're doing is assertion, not aggression. And spend time with other women. I think we spend too much time in competition with other women. Spend time uplifting other women and uplift yourself in that process.

This reflection by Anastasia comes across as a charge to other women student affairs professionals, and alludes to a climate and context issue worth addressing. The following comment by Betty also relates to issues with work environment. As mentioned earlier, Betty's role has involved working closely with men on numerous occasions, and this work has contributed to her current perspective:

I feel like I've been able to figure out how to navigate it [gender] instead of being like, “oh, it's there and it's a detriment." I look at, how do I use it as a strength and try to become more of an ally with men who are in power? And then try to figure out how do I pull up the women with me and educate them?

Betty further explains how she believes her approach helps her understand where the men she works with are "coming from." Particularly in maneuvering conversations, she claims, "if you don't have the relationship [with the men], they're not going to listen to you. And the only way to get the relationship is sometimes, you have to drop some of it [gender] at the door." Betty clarified that while she does not always agree that this type of approach should be her only choice, she takes so that conversations can move forward. 
In the same way that Betty describes various approaches to working with men, Ann's observations of her supervisor have caused her to think more about how gender can be used as a strength:

So, for the age of our director, she's faced many more gender challenges and she shares those experiences with us. She, in many respects, is a woman in a man's world and tries to stake her claim. But then there's often times that I see her modeling male behavior and paradoxical to me. It's interesting how she presents herself when she's trying to buck gender stereotypes. I think midlevel professionals are much more, especially women, are much more comfortable being feminine and showing femininity than are senior level professionals. So, it's just interesting watching the dynamics. And then we'll have conversations with [our supervisor] about "well, put on your suit today.” And we don't like suits. We don't want to wear suits.

Ann's example is important considering both gender and professional level, significant to this study on women midlevel student affairs practitioners.

Gender salience is a common theme among the narratives in this study. While stories related to the types of interactions women have with men in the workplace may not come as a surprise to the readers of this study, they are significant in considering how women represent themselves as women. Attention is devoted in Chapter 6 to how women use gender as a strength in their professional lives.

\section{Theme 6: Similar personal stories}

Likely evident from the themes and examples provided so far in this chapter, the women in this study share professional stories containing several similarities to one another. Examining the similarities closer resulted in three subthemes for this theme. Personal attributes refer to their 
family, educational, and professional backgrounds, as well as the inherent strengths they possess. Work/life balance contains stories of attempting to give adequate attention to work while also preserving their personal life. Approach to professional development summarizes the ways in which they push themselves to grow professionally and move ahead in their career.

\section{Personal Attributes}

Many of the women were quick to credit their family for providing a foundation that supported education. The first image in Anastasia's sketch uses a house to depict her family history. Sitting atop several bricks, depicts the house on a solid foundation. The bricks are labeled with the words family, mentors, and education. There is also a brick labeled TRIO, the acronym referring to three federal outreach and student services programs designed to identify and provide services for individuals from disadvantaged backgrounds, Upward Bound, Talent Search, and Student Support Service (U.S. Department of Education, 2017). Anastasia explains the image:

[I] started with a strong foundation - family, commitment to education, mentors, and TRIO; all the things that pushed me to go where I am and be who I am. [There are] things I couldn't have done without those foundational items. I was first in my family, first gen[eration].

Anastasia went on to explain that she was the first in her family to graduate with an undergraduate degree, then a graduate degree, and now the only person in her family to pursue a doctorate. 


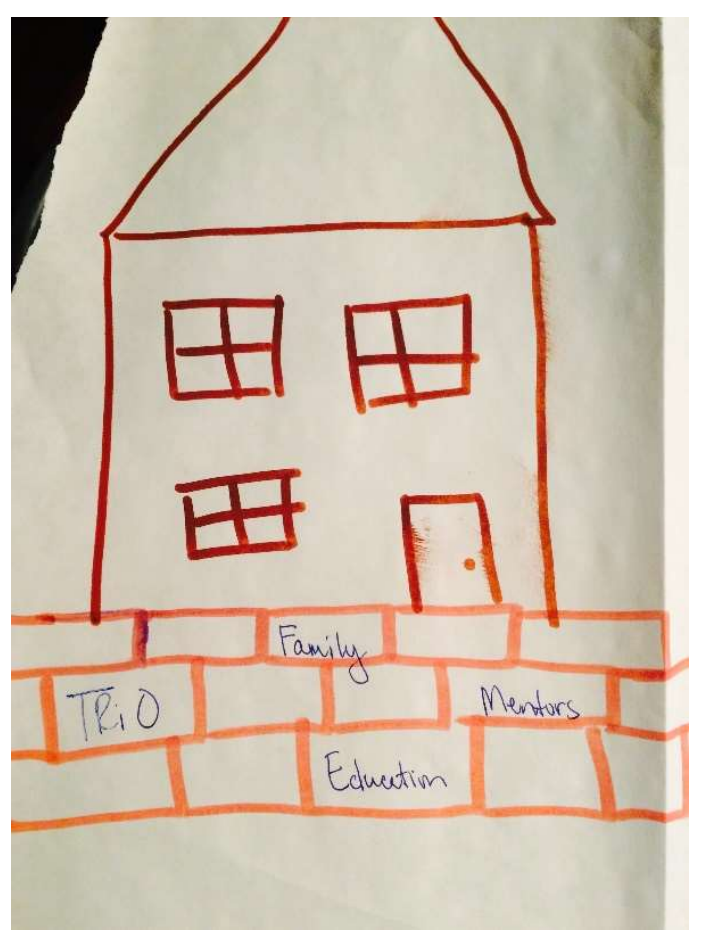

Figure 10. House from Anastasia's sketch

Portraying a distinctly different background, Zoe and Anastasia share a history of education as an important goal, and motivating factor for success.

Zoe is a self-described "fifth generation college educated" individual and shared many stories of influential relatives who not only encouraged her to pursue higher education, but set high expectations for doing so. Many of these individuals are women. She shared that because of the people who surrounded her during her early development "the concept of [academic] success was probably much more mainstreamed in me as a Black woman than my cousins who lived [elsewhere]." Zoe's family is also deeply rooted in service and she comes from "a culture of extreme conscious dialogue.” This environment contributes to her passion for activism and pushing her students and colleagues to think critically, and she often experiences frustration when they do not possess such skills.

When asked about personal attributes that have contributed to their professional success, five of the six women specifically mentioned StrengthsQuest, an online resource that is 
commonly used in higher education to assist individuals in the identification of their top strengths as opposed to weaknesses. For ease in reading the remainder of this section, terms identified as strengths by Strengths Quest will be italicized.

Betty's strengths include ideation. Ann's strengths include adaptability and arranger and she adds that she likes to "put things in order ... know her capacity" in terms of what is willing to take on and what not to. She is trying to be more comfortable in "rolling with it" (perhaps more important now that her life looks different as a working mother). Liana states that she possesses:

An inherent desire and drive to do well, [and has] no desire to do anything half-hearted. I've always been one to just like believe in people, and I think if a person wants to do something, they can do it.

Although Liana did not list her specific strengths, she immediately referenced the StrengthsQuest tool when answering questions related to personal attributes.

Anastasia listed a few strengths, responsibility, accountability, and harmony, and then used her own words to say she is "committed, driven, and gets shit done." Zoe listed strengths that include input, learner, deliberative, and individualization and added that being "laid-back" and having "optimism" has been helpful to her professional story. Finally, Cathy did not mention StrengthsQuest but believes an important personal attribute is her "ability to connect with people and relationship building" as well as her "helping values and wanting to contribute."

Another example from Anastasia's sketch portrays her working on her doctoral degree with a drawing of a person pushing a large ball ahead of them on a very steep incline, indicating her feelings of always working on something and often feeling like little progress is being made: 
So, this is me pushing the boulder up the hill constantly with like little to no movement or growth. Like, this constant. Not knowing, [yet] also completely owning. Doing this and working full time is hard. Doing this while doing social justice work is hard. So, it's [the image is] me constantly pushing the boulder up the hill. That's it.

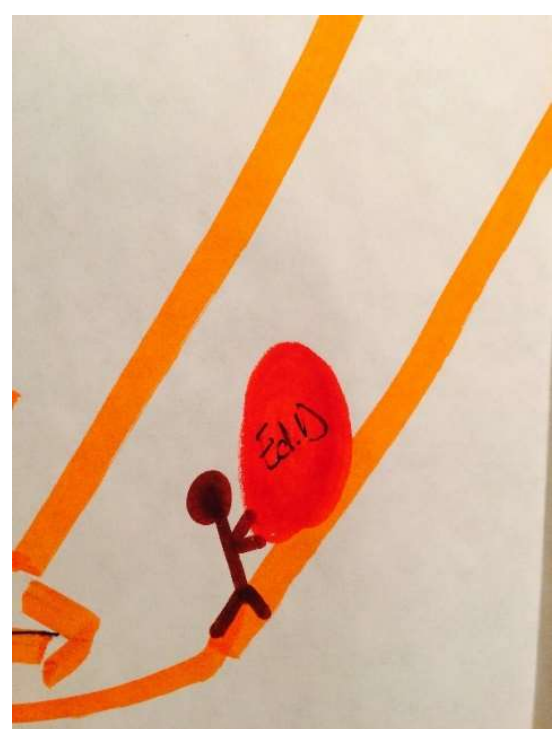

Figure 11. Boulder from Anastasia's sketch

Another time in the interview, Anastasia referenced the boulder as "with little to no movement or growth.” As she talked through her academic experiences, Anastasia and I discussed the numerous challenges associated with completing a doctoral degree, mainly the constant feelings of frustration as we attempt to meet internal and external expectations both personally and professionally.

\section{Approach to Work/Life Balance}

None of the women believe balance is attainable when it comes to prioritizing and juggling the demands of their personal and professional lives. Some have come to this conclusion based on personal experience while others have been influenced by others. Advice that Anastasia received from a professional training is that "it's ebb and flow; it's never a 
balance, it's ebb and flow. So, that's how I refer to it now. There's no such thing as balance of this life.” Betty added a similar perspective:

A woman said, "you will never be able to have true balance in life because you're never going to be able to do everything at once. You can do everything at some point in your life but you can't do it all at once" and I just really kept remembering that.

The impact of the woman's statement has resonated with Betty as she experienced job changes, moves, starting a family, and completing a doctoral degree. Although she has made significant progress towards her long-term career goals, she refers to the expectation to do it all as everpresent.

Anastasia's approach to balance has also developed over time, and has been a true learning experience. Trying new things has enabled her to learn from the past:

Early on, I didn't have a lot of healthy strategies and there weren't any healthy strategies built into the office. It was like we work, work, work, work, work, work, and you're expected to be accessible 24 hours. At some point, I realized that's not how it has to be. So, I set strict boundaries for myself about how I use my time and I have outlets for decompressing. Those are the two biggest things I find that allow me to have that ebb and flow.

A specific example of how Anastasia attempts to prioritize her work time is also a way to keep her connected to the reasons she stays in the profession. "I put lunch on my calendar. Sometimes I work through it, [but] if I can, I sit down and I can engage with students, which gets me back to why I do the work." Anastasia's passion for empowering students is evident throughout her professional story. 
Some women talked about the importance of setting boundaries, and then holding themselves and others accountable. Liana reflects, "I've learned through having two kids that I also need to set time aside for me. So, I get up; I wake up early and work out and that's my time, that's just my time." Ann's story included the commitment she and her partner have made to setting boundaries between work and personal time, and she was honest that the task is not always easy:

I think when we overstep some of those bounds in our personal life with professional things is when we've agreed we're not going to let them intrude and one or both of us still let them intrude. And that is just then we need to recommit to whatever decision we've made and, if it's that we're off the grid for four days and not answering ... it can wait. [It is] an ongoing balance of how to make it all work.

Ann's example of protecting the time when she is not at work is clearly a priority for her partner, as well. Her story has implications for how supervisors convey expectations of their staff and when tasks must be completed.

Cathy's strategy is to be intentional in how she plans her day, and evening hours:

I always try to leave myself a half an hour in the morning to just dig into e-mails. We are on a campus where people are e-mailing each other at night and I, unless it's an emergency, I don't respond to those. I've let people know, if you really need to get ahold of me, it's through a text because I try very much to turn off my e-mail in the evenings. This example of expectations around work correspondence after hours connects back to the struggle Cathy mentioned in Theme 3 pertaining to office norms.

The women also talked about the need for student affairs professionals to advocate for themselves. Anastasia notes that women in the profession have, like her, had to navigate the 
challenge of "how to say no, but also to assert [one]self" and that she is "conscious of how others perceive her." Anastasia reveals key strategies that have helped her:

This work is really difficult, and so I encourage my staff and model myself. If you have access, having a therapist is great. I have a lot of boundaries about how I can show up and how my identities can show up and sometimes I'm not allowed to fully be myself. And so, I go and work through that in therapy.

This advice Anastasia provides indicates she has reached a point in her life where she makes intentional choices in how and where she spends her time and energy. This transitions nicely to the ways the women approach professional development.

\section{Approach to Professional Development}

Regarding professional growth, several of the women mentioned looking at their resume to identify what may be missing, and carefully considering new tasks to take on. Once they have identified an area for growth, they take deliberate steps to try and learn that skill.

Ann recognizes that obtaining new skills is important to her overall career development, "I need to own where the gaps are in my professional background and focus on that." Similarly, Zoe also approaches professional development with her long-term goals in mind. Having a desire to challenge herself, she asks, "What are meaningful things I can look for? What are places that are going to challenge me?" Admitting that she is open to working in other areas of the student affairs field, keeping the bigger picture in mind is helpful to Zoe as she considers if opportunities are worth her time and effort.

Cathy attempts to engage in professional development on a regular basis, providing a unique example "I think about when I look at my week, when I look at the calendar, what scares me? Or, what am I not looking forward to? And I try to generally lean in." Cathy credits a former 
supervisor for encouraging her to fill the gaps on her resume or from her work history. Now a supervisor herself, Cathy employs this strategy with her own staff.

Their characteristics of gender and professional level resulted in these women being a natural fit for this study. However, as themes emerged from their stories, the high number of similarities among the six were thought-provoking. Shared histories and personal attributes seem to have led them to similar points in their careers, and they carry similar approaches to how they balance their time, as well as how they seek to develop themselves as professionals. Perhaps it is their lived experiences that contribute to their willingness to share insight with individuals entering the profession.

\section{Theme 7: Advice for the Future of the Field}

As the women began sharing their professional stories, much of the first part of the narrative included their educational background and training as they were socialized into the student affairs profession. Many positive and meaningful stories were shared, but simultaneously, it became evident there exist some gaps in their preparation. These examples resulted in the first subtheme of this section, administrative tasks, as a nod to what they wish they had been taught. Also, the women spoke freely about what is needed for practitioners to be successful as they move forward in student affairs. The second subtheme, bigger picture, includes suggestions for collaboration and campus buy-in applicable to all student affairs practitioners, as well as advice specific to midlevel professionals.

\section{Administrative Tasks}

Liana expressed her graduate program and assistantship failed to fully address "how to manage time, tasks, and people" and that "administrative functions in student affairs is not talked about as much." Betty had the same experience, "nobody really taught me what the difference 
was between advising and supervising; it took a lot of talking it through and me figuring out the hard way." Anastasia also struggles with the lack of training in this area:

I'm finding I need a lot of help in personnel. No one tells you how much of this work is about the humans. I need allies and mentors in the field who can help me navigate the landmines that I don't even know that I don't know.

Referring to student affairs as a "political game", Anastasia discussed the importance of professionals helping one another, and how she now attempts to do this for others.

Conversations with the women about gaps in their professional training naturally led to them offering advice for new professionals coming into the student affairs field. Several mentioned there is not enough talk within the field about how to link theory to practice. Other topics included concern that new professionals may not possess the practical skills needed to be a successful practitioner. Zoe asks [about new staff] "Can they critically think? Can they speak to someone?" Expressing that simply having a degree related to the field is not enough, Zoe emphasizes that practitioners must be able "to figure out an issue" and overall, "be good humans." Finding herself in conversations with colleagues where she must advocate for this expectation supports the need for alliances to successful do student affairs work.

\section{Bigger Picture}

Additional advice related to how to collaborate, getting buy-in from campus partners and leadership, and how to connect to the bigger picture at an institution and within the larger field. All the women have built a diverse skillset during their time as a student affairs practitioner, and some mentioned that the task was strategic. Betty shared that rather than concentrate on a certain area or functional unit, she continues to look for ways to learn, "I want to do that next level, plus I do like doing more. I didn't want to be a specialist; I want to be a generalist." 
Referring specifically to midlevel student affairs professionals, Ann feels they are not as prepared to move up in administration and encourages her peers not to use current student affairs leaders as their only example of how to navigate career advancement:

We can't use their story as our story because our stories are very different. There's more of us [midlevel professionals] now, [yet] we're still not being strategic about how we make those steps. So, this midlevel group is really interesting because we still don't have it figured out. And I think the younger group that's coming up have a little bit better of a "you do this first, you do this second, this third" and so they're kind of seeing the path. Ann's encouragement is for colleagues to think alternatively to what they already know, or from what they have been shown by more senior professionals.

A similar sentiment in being creative in approaching the future of the field comes from Liana, through her mention of the impact perspectives from other disciplines could have on our work, specifically "organizational change and organizational analysis." From her recent experiences in taking courses in these fields, Liana sees potential in incorporating them into student affairs work.

Finally, Ann sums up her perceptions of where the field is headed through this following advice for her peers:

As midlevel professionals, push forward, 'cause not everybody's asking us to come to the table. [Don't be] afraid to have conversations as a midlevel professional, that next level of knowing succession plans. I think a lot of midlevel professionals are afraid to have that conversation or voice that they're interested. Collaborate in ways that we're actually utilizing all of our resources and being the best stewards of the students that we can be with their dollars. 
Ann's advice could be well-received by practitioners seeking to take innovative paths towards their next position in student affairs. Oftentimes, learning from others who share your similar professional standing is just the encouragement needed to make important career decisions.

Considering changes to the higher education landscape since the student affairs field was created, reflections by the women in this study may be timely and welcomed by colleagues in the field. While the study focuses on the careers of women midlevel practitioners, this theme underscores the potential impact these findings could have on the research, practice, and theories of student affairs.

\section{Additional Finding}

Through the data analysis and identification of themes and subthemes, an additional finding emerged. Although not selected as a main theme, the following depicts the women's reflection on participating in the study, sharing that the process of sharing their personal narrative was meaningful, even cathartic for some.

\section{Sharing their Personal Narrative Included Emotion}

All the woman shared that they enjoyed the process of telling their professional stories and described the experience as reflective. Some became emotional during certain points of their story. Anastasia was instantly transparent about her emotion, "I always cry; I'm not going to cry," while others displayed emotion during or immediately after sharing a piece of their story. Liana began to cry after sharing that she and her partner initially planned to live in an area for a short time but became more connected than they anticipated, "we'll stay here for like 3 to 5 years and then go on to something else; then we really settled into a community and that was really good for us." While she started to offer an apology for becoming emotional, she quickly nodded, indicating she was okay, and appeared comfortable continuing with her story. 
Several of the women referred to the interviews and sketch activity as therapy. Anastasia concluded our first interview by referencing the sketch activity, saying, "this was fun; I might steal this activity from you." Others indicated that after the first interview they planned to call a former supervisor, mentor, or their parents to thank them for their support. Cathy spoke highly of her former supervisor who provided numerous learning opportunities and has remained supportive throughout her journey. After sharing more about the individual, Cathy remarked, "I need to call her tonight and thank her." Cathy later spoke of her close relationship with her family and how they have also provided continual support, "now I need to call my parents tonight, too." I followed up on this comment when I called Cathy for our second interview, and she confirmed that she made both calls.

While not all women cried, or became emotional during the process of participating in the study, it was obvious that sharing such personal moments certainly came with strong feelings, resulting in stirred emotions. In the same way that the women felt that sharing their narratives was reflective to them personally, I believe their stories are equally important to others. Next, I interpret the themes through the parameters of the theoretical framework to make meaning of the information in ways that will impact the student affairs field.

\section{Interpretation of Themes through Theoretical Framework}

It is important to briefly revisit information about the theoretical framework, the Kaleidoscope Career Model (KCM), to provide context to how the themes were interpreted. The KCM was chosen to assist in the understanding of career experiences as told by the participants through the sharing of their personal narratives. A significant part of the literature review supporting this study, the KCM parameters - authenticity, balance, and challenge - informed the interview protocol for the first interview (Appendix C) by guiding the development of individual 
questions. Intentionally omitted from the initial data analysis so that themes could emerge naturally, the second part of data analysis used the KCM parameters to interpret the themes (see Table 3).

\section{Parameters}

As explained in Chapter 2, the parameters are individually defined as:

- Authenticity relates to the need to be true to oneself, genuine, and to align behaviors and attitudes with personal values, perhaps leading them to pursue their own path;

- Balance is the need to satisfactorily manage both work and life/family, and to create a healthy equilibrium among work, relationships, caregiving roles, and other non-work aspects of life, and may result in using strategies such as adjusting their work time through part-time employment, opting out from the workforce temporarily, taking turns to opt out with their spouses, and arranging workloads in accordance with family situations; and

- Challenge is the need to improve oneself, striving for stimulating work (and stimulating assignments on the job), career advancement, and self-worth, and may include individuals who are willing to go beyond at work.

\section{Interpretation Process}

There are two significant aspects to the interpretation process. First, while each of the parameters can stand alone, it is also possible for parameters to coexist. Reflective of a kaleidoscope career, women seldom make career decisions with just one criteria in mind. In fact, the kaleidoscope as a metaphor for a career emphasizes that as women make career decisions, a myriad of factors is considered. Consequently, once the decision is made, it shapes multiple 
aspects of her life, not just her career (e.g., marriage, motherhood, family responsibilities, educational endeavors, friendships, community involvement, and professional development).

Secondly, I employed the use of a table (see Table 3) to indicate which subthemes related to each of the parameters. Since the subthemes make up the main theme, it was only necessary to interpret the former. This activity produced a visualization of the interpretation process, resulting in a format that is easy to understand, and provides a clear framework for the discussion in Chapter 6.

Table 2. Themes interpreted through the KCM

\begin{tabular}{|c|c|c|c|}
\hline $\begin{array}{l}\text { Theme } \\
\text { Subtheme(s) }\end{array}$ & Authenticity & Balance & Challenge \\
\hline \multicolumn{4}{|l|}{ Reluctance to take full credit for career success } \\
\hline Unplanned & & $\mathrm{X}$ & $\mathrm{X}$ \\
\hline Strategic & $\mathrm{X}$ & $\mathrm{X}$ & \\
\hline \multicolumn{4}{|l|}{ Defining success through feelings } \\
\hline Validated & $\mathrm{X}$ & & \\
\hline Motivated & $\mathrm{X}$ & & $\mathrm{X}$ \\
\hline Redefined & $\mathrm{X}$ & $\mathrm{X}$ & $\mathrm{X}$ \\
\hline \multicolumn{4}{|c|}{ Misalignment between supervisor philosophy \& actions } \\
\hline Office norms & & $\mathrm{X}$ & $\mathrm{X}$ \\
\hline Impact on supervisory style & & $\mathrm{X}$ & $\mathrm{X}$ \\
\hline \multicolumn{4}{|l|}{ Importance of mentoring network } \\
\hline Support from same gender & $\mathrm{X}$ & & \\
\hline Unconventional support & & $\mathrm{X}$ & \\
\hline Building a diverse network & $\mathrm{X}$ & $\mathrm{X}$ & $\mathrm{X}$ \\
\hline \multicolumn{4}{|l|}{ Similar view of gender } \\
\hline Culture dominated by men & & $\mathrm{X}$ & $\mathrm{X}$ \\
\hline Representing self & $\mathrm{X}$ & $\mathrm{X}$ & $\mathrm{X}$ \\
\hline Interactions with other women & & $\mathrm{X}$ & \\
\hline Gender as a strength & $\mathrm{X}$ & $\mathrm{X}$ & $\mathrm{X}$ \\
\hline \multicolumn{4}{|l|}{ Similar personal stories } \\
\hline Personal attributes & $\mathrm{X}$ & & $\mathrm{X}$ \\
\hline Approach to work/life balance & $\mathrm{X}$ & $\mathrm{X}$ & $\mathrm{X}$ \\
\hline Approach to professional development & $\mathrm{X}$ & & $\mathrm{X}$ \\
\hline \multicolumn{4}{|l|}{ Advice for the future of the field } \\
\hline Administrative tasks & & $X$ & $\mathrm{X}$ \\
\hline Bigger picture & $\mathrm{X}$ & $\mathrm{X}$ & $\mathrm{X}$ \\
\hline
\end{tabular}




\section{Summary of Interpretation of Findings}

A primary challenge in interpreting themes through the $\mathrm{KCM}$ was a tendency to make the case that every theme and subtheme associated with all three parameters. The broadness of the parameter names allowed room for me to consider potential connections throughout. Had I continued with this approach, however, the result would be confusing and serve unhelpful in framing the discussion of the findings. Reflecting on the purpose of the process permitted me to reexamine the interpretation process more closely, keeping the end goal of this project in mind. It is not conducive to the purpose of this study to discuss at length every subtheme, nor each instance where the subtheme connected with a parameter in the interpretation. Instead, I have identified those connections that most align with the research questions. Below is a brief review of the connecting points.

Authenticity most strongly connects to (a) strategic career decisions; (b) feelings of validation and motivation, and how the women have redefined success; (c) the impact on their own supervisory style when misalignment between a supervisor's philosophy and actions is noted; (d) mentoring from same gender and how their approach to building a diverse mentoring network; (e) similar views of gender in terms of how they represent themselves and use gender as a strength; (f) similar personal stories in terms of personal attributes, approach to work/life balance, and approach to professional development; and (g) advice related to the bigger picture in terms of the future of the field.

Balance most strongly connects to (a) both unplanned and strategic career decisions; (b) how the women have redefined success; (c) how they approach office norms and the impact on their own supervisory style when misalignment between a supervisor's philosophy and actions is noted; (d) mentoring that comes from unconventional sources and their approach to building a 
diverse mentoring network; (e) similar views of gender in terms of a culture dominated by men, how they represent themselves, and how they use gender as a strength; (f) similar personal stories in terms of approaches to work/life balance and professional development; and (g) advice for the future of the field that includes a focus on administrative tasks and looking at the bigger picture.

Challenge most strongly connects to (a) unplanned career decisions; (b) redefining definitions of success; (c) how they approach office norms and the impact on their own supervisory style when misalignment between a supervisor's philosophy and actions is noted; (d) building a diverse mentoring network; (e) similar views of gender in terms of a culture dominated by men, how they represent themselves, and how they use gender as a strength; (f) similar personal stories in terms of personal attributes, approach to work/life balance, and approach to professional development; and (g) advice for the future of the field that includes a focus on administrative tasks and looking at the bigger picture.

Given the substantial amount of connections found through the interpretation, I now transition my focus to the findings most relevant to my research questions. In the next and final chapter, I provide an analysis of these findings in relation to the literature, the significance of the study, and implications for research, practice, and theory. 


\section{Chapter 6: Discussion and Implications}

The purpose of this narrative inquiry was to better understand the careers of women midlevel student affairs professionals. More specifically, the study was guided by two goals: first, to use narrative inquiry and the parameters of the theoretical framework, the Kaleidoscope Career Model (KCM) (Mainiero \& Sullivan, 2005; 2006) to explore how women midlevel student affairs practitioners have developed the competencies needed for career success, paying attention to the role of gender in their career development; and second, to add to the research on career development in the field of student affairs by focusing on a specific understudied population.

Through an in-depth review of existing literature, strategic research design, rigorous methods and analysis, authentic reporting of findings, and a consistent connection to the theoretical framework throughout, the goals of the study were met by:

- exploring the personal narratives of six women, and through their voices, understanding what has contributed to their professional stories, including aspects of career success, and the role gender has played throughout;

- using the KCM to develop interview protocol for the first interview (Appendix C), interpret themes from the findings, and to structure the following discussion;

- providing recommendations for further research on career development and competency development in the field of student affairs; and

- providing recommendations for practice to better support women midlevel student affairs practitioners. 
In this chapter, I begin with a discussion on the significance of the study to the field of student affairs. Then, I address how the findings of this study help to answer the research questions. Finally, I conclude with implications for educational research, practice, and theory.

\section{Significance of Study}

This study contributed to a gap in the literature by taking a new direction in the research of professional competency in student affairs. Within the history of the competency discussion is the noted absence of how competencies are attained, and what the impact of this process is on career development. Rather than using existing formal competencies for the field as a starting point to understand the issue, this study instead allowed information to emerge organically through engaging current practitioners to tell their professional stories, using their own voices. Exploring the ways practitioners already working in the field develop (and have developed) competencies that have contributed to their professional development, professional mobility, and career success is helpful in the field's understanding of how student affairs careers develop to the point where practitioners believe they are successful.

Further narrowing the focus of the study was the selection of a specific subgroup of professionals as the sample. Two identifying characteristics, gender and career stage, resulted in women midlevel student affairs practitioners as the participants of the study. Gender is important primarily because although more women are reaching top leadership positions (Duffy, 2010), there still exists a large number of women in midlevel positions (J. Taylor, personal communication, June 29, 2017). In fact, women in student affairs may be clustered at the middle. As one study revealed, women have been unable to advance in higher education careers with the same "speed, ease, or height of their male counterparts" (Thomas et al., 2004, p. 63).

Additionally, midlevel student affairs professionals have received little attention in recent years, 
with only two noteworthy publications focusing specifically on this group within the last 15 years (Ackerman, 2007; Renn \& Hughes, 2004). It is important for the future career advancement of women student affairs professionals to study those who have chosen to stay in the field; findings from such research will help us know more about competencies, how they develop, and how they might continue to develop.

Taking an interdisciplinary approach to this study served great benefit, specifically the methodological choice and theoretical framework. Given the vastness of the student affairs field in comparison to the narrow focus of this study, the choice to collect personal narratives and build a meta-narrative provides a unique view into the lived experiences of practitioners. Narrative inquiry is useful for researchers interested in empowering a small number of individuals whose identities are critically important to success of the project, relevant to the small sample size of this study. In this study, six women shared their professional stories. The individual narratives had many interesting turns and nonlinear paths but revealed common threads related to the opportunities and challenges, personal decisions, advancements and setbacks, and influences of gender that have shaped their careers. Using a theoretical framework (KCM) from outside of education resulted in a new perspective on what contributes to a successful career in student affairs. Attempting to understand the student affairs career of a woman midlevel professional through the kaleidoscope metaphor resulted in findings that complement our current understanding of career development, yet revealed new information about how women describe their success, and the competencies they believe are beneficial to that success. Thus, the timing and applicability of contributing new information to the competencies discourse is significant. 


\section{Research Questions}

Throughout this study, I sought to answer the following research questions:

1. How do women midlevel student affairs practitioners describe their professional life story, particularly their current levels of success within the field?

a. To what do they attribute that success?

b. What role has gender played?

2. What do their histories reveal about key aspects of their personal attributes, skill development, and professional experiences that might help inform the field's conceptualization of competency development?

Below, I draw across the subsections identified in Chapter 5 to discuss how the findings of this study help answer these research questions. The potential still existed to connect (either formally or loosely) all interpreted findings to the research questions. Instead, I identified those findings that most align with the research questions, as well as provide a pathway to discuss implications for research, practice, and theory.

\section{Professional Life Stories}

My assumption of the transformative power of storytelling was confirmed through the process of conducting this study. As I intently processed individual narratives shared, the purpose of the study served as a consistent reminder to keep the bigger picture in mind, recalling that "meaning-making also comes from comparing stories" (Patton, 2002, p. 478). The metanarrative referred to throughout this study revealed these women possess a desire for authenticity as an aspect of career success, the necessity of balance, and the role gender has played (both supportive and challenging) in the careers of the women who participated in this study. 


\section{Career Success}

My participants appeared reluctant to take full credit for their career success. Rather, they gave a large amount of credit to the other people in their stories. Their stories began with phrases like, "I don't know that I'm a super active participant in this," that a position was "cobbled together" for them, and that things "fell into place" to describe other people providing opportunities. However, when pressed to provide details around their career decisions, each participant shared various ways she had been strategic in asking for additional job responsibilities, adjusting work and family schedules to devote more time to completing a dissertation, and changing jobs to learn different skills. While immediately evident to me, I do not believe these women picked up on the differences between the parts of their stories. Perhaps they have not been given an opportunity to reflect.

Considering the various career stages in student affairs, it would be interesting to compare this study's findings from midlevel professionals to entry-level professionals, and if the latter may be more inclined to admit they are strategic in career decisions, specifically how they approach the initial job search. Linked to previous research that used the $\mathrm{KCM}$ in education, Ervin (2015) found authenticity appeared more important in early career than in more advanced stages. On the other end of the professional spectrum, comparing midlevels to SSAOs or seasoned professionals to see if they are more willing to credit their own strategic decisions due to time in the field. But the women in this study represent the midlevel, described as having no identity (Young, 2007) and it not commonplace in the profession to formally reflect on career decisions. Thus, who would ask them to talk through their stories? Further consideration should be given to the possible benefits of talking through the aspects of how, when, and why choices are made by women midlevel practitioners, as supported by Gagnon's (2010) use of narrative 
inquiry and the $\mathrm{KCM}$ to explore mid-career professionals. Also linked the $\mathrm{KCM}$ literature is an additional thought; practitioners should be encouraged to design career paths that align with their individual goals, similar to Yourston's (2016) findings that a multitude of factors like profession, gender, and life stage should be considered in career decisions.

Regarding midlevel professionals, it is conceivable that some are content at the midcareer stage, and the field should not assume an SSAO position is not the ultimate career goal for all. Thus, this study builds on the work of Renn and Hughes (2004) on women at midcareer, supporting the power of understanding the lived experiences of others. The findings also connect to Belenky et al. (1986), highlighting the valuable insight of understanding constructed knowledge in terms of how women make decisions, and are especially relevant to how a woman's environment shapes her choices around career goals and expectations.

Finally, personal definitions of success are critical for the larger field of student affairs to understand. How women midlevel professionals define success is relevant to their desire for careers that are aligned with their personal values. Across all six narratives, feeling words were used to convey their personal definitions of success. The women midlevel professionals in my study feel validated by experiences in the workplace and comments by colleagues and students, and motivated to continue doing student affairs work. As mentioned in earlier chapters, their non-verbal communication changed (for the positive) as they shared stories that made them feel good about their work. Combined with a desire for balance resulted in many of these women redefining what success looks like at the midlevel. Noteworthy to the field is this redefinition process is done at the individual level, unprompted by anyone else. Their professional level being mid-career is likely a contributing factor to the maturity needed to redefine their definition 
of success, and their years of work experience served as reference in their perspective on what is important.

Like the recommendations made regarding the need for reflection in the field, leaders should also consider how success is regarded by the profession, and subsequently, its effect on professionals at all levels. While the importance of socialization into a student affairs career has been supported (Golde, 1998; Ortiz, 2004; Sallee, 2011), not much attention to socialization seems to be given to this phase, particularly relative to the attention given to graduate students and new professionals. Attention should be devoted to the socialization of specific populations of practitioners.

\section{Need for Balance}

As suggested by the participants in this study, women midlevel practitioners may not receive the support needed to achieve the balance they desire. There is a general understanding of student affairs as a helping profession, and individuals gravitate towards opportunities to support students. Weidman et al. (2001) shared that student affairs staff seek innovative and effective ways to support students, but it does not appear that staff benefit from the same levels of support. These women at the midlevel admit a need to satisfactorily manage both their personal and professional lives, thus influencing how they approach the culture and environment of their student affairs offices and departments. The blurred line between a practitioner's personal and professional life could be attributed to the type of tasks they are asked to perform, as well as the often-fluctuating work schedule required to complete the work. Challenges associated in managing work demands links back to Joyce-Brady’s (2004) story in Roads Taken of an often overwhelming perception by women midlevel professionals to do it all. When imbalances among aspects of their lives are noted, these women employ strategies towards better 
alignment. The assertion that perceptions of work life balance directly relating to gender and career stage is supported by previous literature using the KCM like the study by Aprianingsih (2012) which found the same connections, and builds upon the work of Tajlili (2015) in her study of college women's' attitudes on work life balance.

Personal career decisions often led the women in this study to pursue their own paths, even when their work culture may emphasize something different. As a society, and certainly within our field, the expectation to be always be available by e-mail results in practitioners feeling as they are always working. Although difficult, some of these women have made intentional steps to break this cycle to protect their personal time, echoing the story by Marshall (2004) in Roads Taken regarding strategies employed by working mothers. But supervisors add challenge to situations when there exist misalignments between what they say and do. This notion of misalignment is important for the field to consider. The long-term effects of a work environment plagued by mistrust and low morale could result in an overall lack of commitment to the institution and the field (Schaufeli \& Enzmann, 1998). The women midlevel professionals in this study admit that such experiences have influenced their own supervisory style in that they replicate the positive modeling they have received by their own supervisors. Relatedly, these women adamantly noted their intentions not to replicate their negative experiences with supervisors. Lastly, situations of supervisor misalignment also connect to how and where the women in this study seek support, employing the strategic selection of mentors to fill in the gaps of what they are missing in the workplace

Relationships with other women are important, and I would be remiss not giving adequate attention to how the participants in this study indicated their use of gender as a strength. The positive effects of women being supported by colleagues of the same gender is prominent in 
their stories, and in turn, influential as they deliberately build their diverse mentoring networks. Given the many hurdles that exist in women's career development (Hirsch \& Berman-Hills, 2004), mentorship can serve as a crucial ingredient to assist women in navigating the opportunities and challenges of a student affairs career, and strengthen their connection to the field. Though multiple professional development opportunities for women exist within higher education and student affairs, a closer examination is needed to ensure women midlevel needs are being met.

The concept of a mentoring network (Higgins \& Kram, 2001) is valuable to these women midlevel participants, and they are intentional in connecting with various individuals able to provide support both professional and personal in nature. Examples provided in this study included support from staff they supervise, colleagues from other institutions, and mentors who share their identities in terms of race and gender is valuable. An alternative to only having mentors of the same gender, an unconventional strategy mentioned by a few of the participants is the support they received from men who were colleagues in the field, or and some who reported to the women. Accordingly, the concept of a mentoring network should be explored by leaders at the institutional level to foster intentional connections between women and other campus partners, and by the professional associations in student affairs in order to encourage connections between women working at different institutions and occupying different professional levels, to name a few. Support for such concepts is found by previous KCM literature which asserts networking goals are associated with all three parameters - authenticity, balance, and challenge, and also by the Olyha's (2004) story in Roads Taken which told of the positive impact of a women's mentoring network on her own career. Additional ideas are included in the implications. 
According to stories shared by participants in this study, the student affairs field may not be making women midlevel professionals feel valued. Connected to my earlier points of these women midlevel participants being reluctant to take full credit for their career success, and my assertion that the student affairs field needs to encourage reflections, these women gave different reasons for doing student affairs work. When asked to describe what keeps them in the profession, two women replied they are unsure what else they would do, perhaps indicating a concern they would not be valued in another field. I was struck by the fact two participants provided that exact same answer (verbatim) and am curious if their supervisors and leaders are making them feel valued at work. A third participant answered, "there is still work to be done" as her reason to come back each day, and while this statement indicates a personal passion for her job, I am also curious if the strides she has made through her work to empower students is appreciated by her campus, thus contributing to her feeling of value. Yet a difference in answers is how one woman immediately articulated a clear professional development plan that involves applying for a job annually as way of ensuring she is still marketable. How her statement connects to feeling valued in her current position is unclear, but she obviously has a desire to gauge her value by those outside her institution. What contributes to the stark difference in these examples is critical for the field to explore as institutions dare they risk retaining women who have reached the midcareer point (Wilson, Liddell, Hirschy, \& Pasquesi, 2016).

\section{Role of Gender}

There is a climate and context issue regarding the gender salience noted by this group of women midlevel professionals, and the ways they have (or not) felt empowered to address related issues. In telling the stories of their lives, women frequently recount emotions, experiences, and role identities that match gendered expectations put forth by the dominant, 
patriarchal society (Bloom, 2002). Likewise, the women in this study freely shared the challenges in navigating a field they perceive as dominated by men, including their frequent encounters of gendered situations and the gendered components of leadership.

A general acknowledgement of gender bias is clear through the women's stories in this study, whereas there is less mention of blatant gender discrimination within the field than would be expected from previous decades (Cortina, 2008; Dipboye \& Halverson, 2004). When facing such bias, participants have pursued various ways to represent themselves as women midlevel professionals, particularly related to language, non-verbal cues, and assignment of duties. Examples provided in the narratives indicated divergent approaches. Several stories stressed the uneasiness associated in determining how to react to gender bias, including an assumption that addressing the behavior would be considered confrontational. This point relates to existing research regarding gender bias and microaggressions (Nadal, Meterko, Vargas, \& Wideman, 2016). In contrast, other women adopted strategies they believed were helpful in ensuring their voice was heard. Even though these women admit it feels uncomfortable to do so, some strategies include women consciously aligning their language and behavior with that of counterparts who are men, as a way of building rapport. As a field, what barriers have been created that discourage women from addressing these concerns? Such barriers can push women to pursue entirely different opportunities, as found in previous literature that used the KCM. Braches (2015) found that gendered elements found in more traditional careers influenced some women towards entrepreneurship rather than remaining in hierarchical organizations.

With regard to supervision, a noted challenge is the issue of some of these women feeling unsupported by supervisors, particularly in their need for work/life separation. Of interest to the misalignment between a supervisor's stated philosophy and the actions they display is when the 
supervisor is, in fact, also a woman. There are several examples among the narratives in this study where women supervisors blurred the lines between friendship and reporting structure, exhibited unrealistic and unfair expectations in terms of how and when staff must respond to work issues, and exhibited poor modeling in terms of healthy approaches to work/life balance. Participants shared that they felt conflicted and even let down by women supervisors, and such examples were more prevalent than stories about supervisors who men. Further study of these negative experiences related to women supervisors is intriguing, and connects to the field's shortfalls in preparing supervisors, which will be addressed in the next section.

\section{Conceptualization of Competency Development}

These women midlevel practitioners used a combination of learned skills and inherent

personal attributes and strengths in their daily student affairs work, as well as their overall career. These women's stories indicate they approach skill development using strategies such as asking for additional job responsibilities and making career changes that will enable them to broaden their skillset. They are aware of what their resumes reveal about what they have yet to do, thus consistently engage in opportunities to engage in the professional development needed to learn new skills and obtain experiences needed to fill any gaps.

The student affairs field seems fixated on a formal list of competencies needed to guide practitioners towards being successful in the field (ACPA, 2010a; ACPA, 2010b; ACPA/NASPA, 2015; Burkard, Cole, Ott, \& Stoflet, 2005; Cuyjet, Longwell-Grice, \& Molina, 2009; Hoffman \& Bresciani, 2012; Renn \& Jessup-Anger, 2008).; however, the women in this study relied more on experiences. They shared meaningful stories of times they felt successful and noted the impact of their work on others. Existing research on competencies in student affairs consistently revealed that helping skills are important, directly linking to the words these 
women used to define success (e.g., make impact on others, quantify work as positive change, serve as a role model). Also consistent is how these women describe what they believe to be the personal attributes they claim to rely upon as they make career decisions (e.g., adaptable, accountable, and a desire to do well). Perhaps more examination of student affairs professionals as individuals would be advantageous to the career advice they receive. For example, how do these personal attributes differ from competencies and how do the two concepts relate?

Is the field preparing professionals to be successful in all career stages, but especially by the time they reach mid-career? Is this the role of master's programs, doctoral programs, or ongoing professional development? These women at the midlevel disclose gaps in their own career preparation and offer insight to what the field could do differently. This comparison is analogous to the inconsistencies found between entry level professionals' perception of their preparedness with their supervisors' evaluation (Cuyjet, Longwell-Grice, \& Molina, 2009). Essentially, the participants in this study can easily identify what they were not taught, specifically related to supervising, administrative tasks, and campus politics. Consistently, these women's stories connected these gaps to the advice they provide new professionals. The results of this study questioned whether the field makes women midlevel practitioners feel valued based on the contrasting responses some women provided for their reasons to remain in the field. Investing more in career guidance across all professional levels could be helpful in the long-term professional development of our practitioners, a suggestion applicable to many entities, but to start, graduate preparation programs and leaders of our professional organizations.

From my findings, there appears to be a pattern of ignoring the midcareer voice when studying competencies in student affairs. Existing research on competencies largely overlooked the midlevel professionals, and gender does not appear to be a factor, either. The focus has 
largely been on new professionals and SSAOs. In fact, the midlevel was severely underrepresented on the ACPA/NASPA joint task force that created the Professional Competency Areas for Student Affairs Educators (ACPA/NASPA, 2015). Although these formal competencies did not play a large role in this study, the work is related. This study took a unique angle in approaching the concept of competency development. Narratives included what actual practitioners believe have contributed to their success, thus furthering our understanding of how competencies are developed, rather than producing a list of competencies. It was an intentional decision to not bring up the formal competencies with my participants until the end of the data collection. In fact, the term competency was not mentioned within correspondence about the study, nor in the first interview.

I provide one connection between this study and the Professional Competency Areas for Student Affairs Educators (ACPA/NASPA, 2015). As a closing conversation during second interviews, I asked the participants to talk about their familiarity with the document. All the women in this study acknowledge they have seen the formal competencies at some point either at a professional association or through their graduate preparation program. All the women in this study also shared a reluctance to use the competency document because they have not been shown how. The competencies do not appear to be discussed by the student affairs leaders on their respective campuses, nor in a practical way at professional development settings (professional associations). I shared the electronic version of the document with each participant, and upon review, they identified more than one competency that aligns with their work. Among the competencies mentioned most by of these women were Personal and Ethical Foundations (PPF); Values, Philosophy, and History (VPH; Organizational and Human Resource (OHR); Leadership (LEAD); and Social Justice and Inclusion (SJI). The extent to which these 
competencies hold relevance to these women should be explored more deeply, and is discussed more in the implications for practice below.

\section{Implications}

Using the above section as a foundation, I now apply these findings to the larger field of higher education and student affairs. Although this study focused on a specific population, women midlevel student affairs practitioners, the findings will be useful to individuals, groups, institutions, and professional associations within the field. As a conclusion to this study, I offer several implications for research, practice, and theory.

\section{Research}

Findings from this study lend support to subsequent studies focused on the career development in student affairs, as well as competency development. While this study barely scratched the surface in terms of the role of gender in these women's careers, gender as a strength (a subtheme of Theme 5) is noteworthy due to the different ways it emerged from the participants' professional stories. In her own studies, Gilligan (1982) sought to represent a different voice, "that the way people talk about their lives is of significance, that the language they use and the connections they make reveal the world that they see and in which they act" (p. 2). Therefore, potential studies using discourse analysis (Gee, 2011) or a feminist theoretical lens would be interesting ways of focusing on the language used by women when describing their experiences. In addition to language, studying the concept of gender performance (Goffman, 1959; West \& Zimmerman, 1987) and gender performativity (Butler, 1993) in relation to women in student affairs could provide a better understanding of women's adjustments to behavior based on gender, indicated by some examples shared in this study. 
Formally supporting what many in the field already knew, findings from this study accentuate that additional empirical research related to competencies is needed. During the conceptualization of this study, one idea was to map the experiences shared by participants back to the Professional Competency Areas for Student Affairs Educators (ACPA/NASPA, 2015). While the activity was eventually not included, there still exists potential for use in a separate study. It may also be applicable to use the document as a conceptual framework or data analysis tool. There is currently no research on how the Professional Competency Areas for Student Affairs Educators (ACPA/NASPA 2015) are being utilized in general, or at midcareer specifically, although anecdotal information suggests they are being used by graduate preparation programs to map coursework and integrated into new staff training by divisions of student affairs. Of additional interest is to compare the personal attributes these women midlevel professionals described as important to their decision making with the competencies they believe have contributed to their success in the field. More formal explorations of these concepts would be useful to practitioners, departments, units, divisions, and subgroups within the professional associations who authored both the original version of the document (ACPA/NASPA, 2010a) and the current version (ACPA/NASPA, 2015).

Linked to professional development is that of skill development, and this study revealed barriers related to the preparation of practitioners entering the field, as well as those more seasoned. Findings from this study corroborate an early study on perceived skill deficiencies among graduate preparation programs (Cooper, Mitchell, Eckerle, \& Martin, 2016) as well as studies that stress the connection between curriculum and skills needed in the profession (Cilente et al., 2006; Renn \& Jessup-Anger, 2008). Rather than limiting the scope of such studies to faculty and administrators, more attention in the literature should be focused on interviewing 
current practitioners to find out what skills they lack and how they may be obtained. As indicated by this study, midcareer professionals would be an ideal group to talk with first.

This study exposed gaps in how student affairs literature has omitted some stories, discovering that some voices are still waiting to be heard. Some of my findings link directly to populations within student affairs with valuable insight to share, yet are underrepresented in the scholarly work of our field. The work-life balance theme is a reason to study practitioners with dual responsibilities in addition to their student affairs work, such as parenting, caring for aging parents, and academic pursuits (e.g., dual-career couples in student affairs, practitioners with children, and practitioners embarking on a doctoral program as either part-time or full-time students). The theme noting misalignment between supervisor philosophy and actions suggests that studies about supervisory styles in student affairs, the importance of a supportive work culture, and relationships between supervisors and their staff are needed. Perhaps related to this topic is research on professionals who started in student affairs but left the field at mid-career. The more we learn about individual stories, the better the field can support professionals in meaningful ways.

\section{Practice}

The findings of my study suggest several practical implications for the student affairs field. While the findings revealed key themes from the experiences of women midlevel practitioners, they imply suggestions for others who interact with this population. The field needs to focus on teaching professionals how to supervise, and more clearly articulate the differences between advising and supervising. The women in this study were frank in sharing their frustrations with supervisors of all genders, yet the idea of women supervisors who were unsupportive to women staff was undoubtedly a concern. Staff pay close attention to what they 
do and do not like about how they are supervised, and they adjust their own supervisory style accordingly. Remarkably, the participants list supervising as one of the primary skills they were not taught by either their graduate program or while working in the profession. Faculty should revisit how the topic is introduced in the graduate preparation programs and through graduate assistantships, and student affairs leaders should offer additional training throughout the progressive professional levels. Revisiting Tull's (2006) work on synergistic supervision could be a guide for helping supervisors and staff cooperate within the relationship. Complementing this focus on teaching supervision are implications for how supervisors convey expectations to their staff and when tasks must be completed, and the pressing need for more distinct expectations for work/life balance.

Regarding mentoring, the individual women in this study appear to have a need for building diverse network of support, and are taking on the task themselves. It is pertinent to revisit how the field encourages, discourages, or ignores mentoring, particularly for women practitioners, midlevel practitioners, and those who identify as both. If student affairs is truly a helping field, as some of the participants suggested, administrators and supervisors should encourage mentoring through formal and informal opportunities. Assessment is needed of the existing professional development options for women that are supported by our professional organizations to gauge their effectiveness and to ensure they are inclusive. On the campus level, the strategy shared by one participant of asking colleagues, leaders, and campus partners to coffee for informational interviews could be an easy first step. This is similar to the candid conversations practice offered at the NASPA annual conference, yet the program is limited by time, space, and little potential for the development of lasting relationships. The possibility of mentoring being viewed as gendered is also interesting, contributing to the need for more 
intentional networking to build unconventional relationships. Finally, since midlevel could be a stopping point for some professionals (Donaldson \& Rosser, 2007) the field needs to talk about how that is okay, and provide appropriate mentoring and professional development opportunities that are not aimed at career advancement.

Along the lines of professional development, the Professional Competency Areas for Student Affairs Educators (ACPA/NASPA, 2015) appear to be well received by women midlevel practitioners in this study, likely due to their desire for professional development. Since all the participants indicated a desire to apply the document to their own career development, this study urges the leading professional associations to continue the development of rubrics and guidelines. Related to the larger competency discourse, this study emphasizes the value in understanding individual competency development. Formal competencies now exist for the field, but this does not mean the concept of competency development is fully understood. Practitioners can share their own accounts of the experiences and competencies that have contributed to their success, and efforts to gather and report such information back to the field should be coordinated by student affairs administrators and the professional associations.

Although the student affairs field has experienced growth and change since it was first established, we must question if we are paying enough attention to individual practitioners. Many topics are addresses by the professional organizations, whose leadership is primarily comprised of SSAOs. Even task forces, working groups, and committees are largely spearheaded by efforts at the national level. There are many topics worth revisiting through the examination of individual's professional stories, and unless intentional effort is made, one does not have direct access to others' experiences. Thus, our field risks oversimplifying issues if discussions are kept at surface level, disconnected from actual practitioners. As eluded to earlier in this 
study, there is emancipatory potential of individuals sharing stories (Elliott, 2005). A noted additional finding from this study - for some of the women, sharing their lived experience was emotional. Specifically, the process of using the sketch activity to visually portray their professional story and then using this depiction as a guide to verbally walk through each step in their path required them to revisit the past. In doing so, they revisited challenging times, exciting moments, and the various decisions they (or others) made with regard to their individual career. Thus, some participants shared emancipatory statements about the experience that it was therapeutic, cathartic, enjoyable, and in one case, encouraged one woman to express appreciation to influential mentors in her own story.

Related to gender representation within higher education and student affairs, how are we talking about gender, or are we at all? The women in this study shared examples of not feeling comfortable addressing concerns, feeling their work environment is not conducive to discussing issues of gender bias, work/life balance, and poor examples set by supervisors, contributing to my earlier claim of a climate and context issue. Supervisors and administrators should encourage reflection; practitioners at all levels should be asked to reflect regularly on their professional stories, career decisions, definitions of success, whether their work aligns with personal values, and if the field makes them feel valued as individuals. The KCM is a helpful framework based on the applicability of its three parameters to the career of a woman student affairs professional. Perhaps practical use of the kaleidoscope career metaphor, either formally or informally within student affairs, could assist our understanding of complex careers.

\section{Theory}

To my knowledge, this is the first study within student affairs to use the KCM as a guiding theoretical framework. The application of the framework to this study was an agreeable 
fit based on how the findings aligned with the KCM's parameters of authenticity, balance, and challenge. Within the data analysis, the process of interpreting the themes through the parameters resulted in many connections. As evident in the results (see Table 2), I found support for all the themes across the parameters, but not all the subthemes. While there was an initial tendency to make all fit, several potential connections did not make sense. This revelation reinforces that while the $\mathrm{KCM}$ is beneficial in recognizing the complexities of individual career development, careers are indeed complex, and may not fit easily into parameters or frameworks. The nuances of this interpretation process also support the use of thematic coding to respect the individuality of data versus using the KCM to deductively code. Thus, the choice to thematically code first, then interpret emergent themes through the KCM as a secondary analysis worked in this study, and could serve as an example for subsequent studies.

Comparing this study to previous research that used the KCM, my findings complement how it has been applied to examine gender differences in career perspectives (O'Neil, Bilimoria, \& Saatcioglu, 2003; Powell \& Mainiero, 1992) and the uniqueness of women's careers in comparison to those of men (Mainiero \& Sullivan, 2005). Regarding the few studies within education that have used the KCM, the focus of my study was similar in that they all examined a specific population (Carrahar, Crocitto, \& Sullivan, 2014; Ervin, 2015; Tajlili, 2015). The potential for additional student affairs use of the $\mathrm{KCM}$ relates to its proven helpfulness in studies about retaining women (Mainiero \& Sullivan, 2008) and family friendly environments (Mainiero \& Sullivan, 2007), both topics of interest to the field.

While I have no suggested modifications to the actual theory, I recommend a clearer understanding of its components for it to be most useful to student affairs. For example, the kaleidoscope career is often referred to as boundaryless, yet the absence of such a term within 
the student affairs field may make it a difficult concept for practitioners to understand. Even if they agree with the potential to make career decisions based on authenticity, balance, and challenge, what would those changes look like in our field? How does a professional talk with their supervisor about a desire for such a career? Additionally, authors of the KCM have also created a questionnaire called the Kaleidoscope Career Self-Inventory (KCSI) to help professionals determine what type of career they are motivated to pursue. The KCM refers to the careers as alpha and beta, more easily explained as traditional and flexible. Yet again, simply knowing what type of career one wants is much different than the possibility of it coming to fruition. More examples of how professionals have applied the $\mathrm{KCM}$ and its inventory to their careers in successful ways would be beneficial.

Finally, this study combined narrative inquiry and the KCM to examine careers of women midlevel professionals. Given the link between lived experiences and the time needed to support the reflection needed to consider the aspects of career decisions made over time, this approach may also be useful in understanding the careers of women SSAOs. Within the scope of a student affairs career, SSAOs have accumulated years of work experience that likely encompass various jobs, innumerable relationships, possible institution moves, and major life decisions. Collecting their personal narratives and interpreting through the KCM parameters could illustrate how one decision simultaneously affects multiple aspects of one's life, further the field's understanding of yet another aspect of career development in student affairs. 


\section{Conclusion}

This study advances the student affairs field's understanding of career development and how the field conceptualizes the competency development associated with career advancement and success, particularly of women midlevel practitioners. The research is timely given current discourse on competency development, the release of formal competencies, and the ongoing focus of professional development of practitioners. Although the study focused on a specific population, the findings are applicable to the entire field.

Highlighting the limited research on women at midcareer, and perhaps the previously unknown potential of knowing them better, findings from this study point to the benefits of understanding practitioners as individuals. More than simply examining decisions are considerations of how such decisions are made, particularly related to the gendered environments that permeate student affairs environments. Throughout the chapters, the key terms of voice and lived experiences are intentionally repeated to elevate their importance, as well as provide a consistent connection to the focus and significance of the study.

Finally, the benefit of taking an interdisciplinary approach to study complex issues shown by this study serves as a recommendation for future work. Narrative inquiry as the method and the Kaleidoscope Career Model as the framework enabled me to attend to the multiple interests of this study. I could focus on the importance of the individual, as well as the importance of gender, yet still acknowledge that career decisions impacted by many factors in mind. This study

showed me that competency development is a combination of what is inherently part of a person as well as what is learned. Throughout, I was encouraged by the potential impact of women sharing their own stories to influence change in the field. 
My reaction to this meta-narrative is to be inspired. I ended every interview by telling the women, "if this were my story, I would be proud," and I believe these acts of listening, understanding, and encouraging reflection is what we need more of in the field. As a selfdescribed practitioner-scholar in higher education and student affairs, my personal connection to this study motivated every decision and resulted in a renewed passion for this work. A narrative researcher once said of her personal connection to her study, "if my silence is to be broken, the first [person] I must interview is myself”' (Theobald, 1999, p. 21), emphasizing our need to understand people and their stories. By way of this insight, I asked these women, tell me your professional story. It is my hope that this study will inspire women midlevel practitioners to tell their stories, and for our leadership on campuses and within the field to listen. 
Appendix A

E-mail Invitation Protocol

Dear

You are invited to join a research study titled Women Midlevel Student Affairs Practitioners: Exploring through Narrative Inquiry the Skills, Abilities, and Expertise Needed for Career Success. You have been identified as a potential participant based on your professional reputation and the fact you adhere to the criteria established for ideal participants in this study.

I am a doctoral candidate in the Educational Leadership \& Policy Analysis department at the University of Missouri. In this qualitative research study, I am investigating the experiences of a particular population of student affairs professionals, women, midlevel practitioners. Through narrative inquiry, participants will talk through their professional life stories, and what competencies, skills, experiences, and personal attributes they believe have contributed to their individual career development, as well the role gender may have played.

\section{WHAT IS INVOLVED IN THE STUDY?}

If you decide to participate you will be asked to participate in two interviews; one in-person interview and one interview via Skype or Facetime.

Prior to the first interview, five things will occur:

1. I will e-mail you an informed consent form that explains the full details of participating in this study. Once you have reviewed the informed consent form, please let me know if you have any questions and if you are ready to participate in the study.

2. Next, I will e-mail you a link to an electronic survey to collect information related to demographics, educational experience, current professional/functional area of employment, and years of professional experience in student affairs, and more.

3. I will also collect your current resume or curriculum vitae to assist in my preparation for the interview.

4. We will schedule the first interview to take place in January or February of 2017. I will make plans to travel to your location.

5. Prior to my arrival, I ask that you use a large sheet of blank paper and pens/markers to depict your professional story through drawings and text. This activity is adapted from Nyquist (1999), therefore I will e-mail you this particular publication to provide a better understanding of the task and how the drawing will provide guidance during our subsequent conversations. Take as much time as you would like to complete this activity prior to our first interview. 
The first interview is in-person and will take place at the time and location of your choice. It is estimated the first interview will last approximately 90 minutes. The second interview will be conducted via Skype or FaceTime as a follow-up to the first interview. It is estimated the second interview will be less than an hour, but will provide the participant as much time as needed to add new information or clarify information previously shared in-person.

This study contributes to the research on career development in the student affairs profession by focusing on a specific, understudied population. Implications from this study may reveal key aspects of professional development applicable to individuals and organizations. Participation in this study is voluntary.

I appreciate your consideration of my request. Please feel free to contact me if I can provide additional information about the study and answer any remaining questions you may have. Should you be interested in participating in the study, our next step is to arrange a phone call so that we may schedule the in-person interview.

Thank you,

Laura A. Page

Doctoral Candidate | Graduate Research Assistant

Department of Educational Leadership \& Policy Analysis

University of Missouri | Columbia, MO 65211

573.355.1866 | laurapage@mail.missouri.edu 
Appendix B

Preparing for First Interview

Dear

Thank you again for your willingness to participate in the study titled, Women Midlevel Student Affairs Practitioners: Exploring through Narrative Inquiry the Skills, Abilities, and Expertise Needed for Career Success. I look forward to our interview, scheduled for , from ___ in __. To assist in preparing for the interview, I request that you complete the following tasks, at your convenience.

1. I have created a brief pre-interview questionnaire to collect information regarding participant demographics. The survey may be accessed using this link: Demographics $\underline{\text { Survey }}$

2. I ask that you e-mail me a copy of your current resume or curriculum vitae (CV). Reviewing this document will assist in the preparation of interview questions.

3. Finally, I would like to remind of you the "homework" drawing that I request you complete prior to our first interview. This activity is adapted from Nyquist (1999), and I have attached the publication to this e-mail message. Although Nyquist used this activity with graduate students, I believe it is applicable to helping my participants think through their professional career path, up to the current time. It is not necessary that you read the full article, but skimming portions of it will provide a better understanding of the task and how the drawing will provide guidance during our subsequent conversations. The activity asks that you use a large sheet of blank paper and pens/markers to depict your professional story through drawings and text. There are no set expectations for what this will look like, and examples are included in the article. Take as much time as you would like to complete this activity prior to our first interview.

Feel free to contact me if you have any questions or concerns about the pre-interview questionnaire, the resume/CV request, or the homework activity.

Thank you, and see you on the

Laura A. Page

Doctoral Candidate | Graduate Research Assistant

Department of Educational Leadership \& Policy Analysis

University of Missouri | Columbia, MO 65211

573.355.1866 | laurapage@mail.missouri.edu 


\section{Appendix C}

\section{Interview \#1 Protocol}

- Talk me through your professional story (use sketch as a starting point).

- Reflecting on the different aspects of your work, what parts do you find meaningful?

○ How does your work align with your personal values?

- Regarding your personal and professional life, how do you set priorities?

○ What strategies have worked?

- Who are the people who you engage when making changes to your career?

- Tell me about your professional growth? (listen for specifics to emerge and follow-up).

- How do you identify areas for improvement?

○ How do you identify professional development opportunities?

- How do you define success?

- Tell me the key experiences you felt made you successful. Perhaps have them talk through some examples - a story when they felt really effective/in the flow.

- What do you need to know or be able to do to be successful in your position?

○ Talk to me about what you did yesterday.

- (If gender has not emerged in the conversation, ask)... How has gender played a role in your career?

- Finally, what keeps you in the profession? 


\section{Appendix D}

Member Checking and Second Interview Scheduling

$\mathrm{Hi}$

I have your transcript ready for your review from our first interview. Sending this to my participants is part of the member checking portion of my study. It is completely optional to you if you want to edit, and please feel free to let me know if I misinterpreted a point you made, if I need to clarify, or if you have something to add to a particular section. As a reminder, this transcript will only be seen by me as part of my coding of data.

In addition, I would like to schedule a second interview. If you are willing, this would be no more than 30 minutes and would be conducted over the phone. After I completed my first round of interviews will all participants, I realized there are just a few more specific questions I would like to go back and ask.

Please let me know if this is something we could schedule for the week of Thanks so much,

Laura A. Page

Doctoral Candidate | Graduate Research Assistant

Department of Educational Leadership \& Policy Analysis

University of Missouri | Columbia, MO 65211

573.355.1866 | laurapage@mail.missouri.edu 


\section{Appendix E}

Preparing for Second Interview

Dear

I look forward to our second interview tomorrow, . I will call you at at I am planning on half an hour for our interview.

To make the best use of our time together, I have attached my list of second interview questions. It is possible we addressed some of these points in our first interview, but I am using standard questions for all my study participants for consistency. In addition, I have attached a copy of the ACPA/NASPA Professional Competency Areas for your review, as it may be helpful as you answer one specific question on the list.

Please note that we may not have time to work through all the questions on the list, so let's be sure to talk about those that strike your interest the most.

Thank you so much for making the time for this important conversation!

Laura A. Page

Doctoral Candidate | Graduate Research Assistant

Department of Educational Leadership \& Policy Analysis

University of Missouri | Columbia, MO 65211

573.355.1866 | laurapage@mail.missouri.edu 


\section{Appendix F}

\section{Interview \# 2 Protocol}

- Professionally, what scares you, or challenges you?

- Perhaps consider areas you'd like to develop but have been hesitant or haven't been provided the opportunity yet.

- Thinking of your supervisors, how did their philosophies and actions align (or not)? Example...did they say one thing but not actually display it?

○ Does your answer differ depending on if the supervisor was male or female?

- What could the field have done differently to prepare you?

- What could the field do now?

- What do you believe are some of your strongest personal attributes or skills that have contributed to your success?

- Thinking of the ACPA/NASPA Professional Competencies (attached) which do you relate to most? Relate to least? 
Appendix G

IRB Consent Form

\section{INFORMED CONSENT}

You are invited to join a research study titled Women Midlevel Student Affairs Practitioners: Exploring through Narrative Inquiry the Skills, Abilities, and Expertise Needed for Career Success. Your participation in this study is voluntary.

I am a doctoral candidate in the Educational Leadership \& Policy Analysis department at the University of Missouri. In this qualitative research study, I am investigating the experiences of a particular population of student affairs professionals, women, midlevel practitioners. Through narrative inquiry, participants will talk through their professional life stories, and what skills, experiences, abilities, and expertise they believe have contributed to their individual career development, as well the role gender may have played.

You have been identified as a potential participant based on your professional reputation and the fact you meet the criteria established for ideal participants in this study.

WHAT IS INVOLVED IN THE STUDY? If you decide to participate you will be asked to participate in two interviews; one in-person interview and one interview via Skype or Facetime.

Prior to the first interview, the following steps will occur:

6. Please review this informed consent form in its entirety. Let me know if you have any questions, and if you are ready to participate in the study.

7. Next, I will e-mail you a link to an electronic survey to collect information related to demographics, educational experience, current professional/functional area of employment, and years of professional experience in student affairs, and more.

8. I will also collect your current resume or curriculum vitae to assist in my preparation for the interview.

9. We will schedule the first interview to take place in January or February of 2017. I will make plans to travel to your location.

10. Prior to my arrival, I ask that you use a large sheet of blank paper and pens/markers to depict your professional story through drawings and text. This activity is adapted from Nyquist (1999), therefore I will e-mail you this particular publication to provide a better understanding of the task and how the drawing will provide guidance during our subsequent conversations. Take as much time as you would like to complete this activity prior to our first interview. 
The first interview is in-person and will take place at a time and location of your choice. It is estimated the first interview will last approximately 90 minutes.

The second interview will be conducted via Skype or FaceTime as a follow-up to the first interview. It is estimated the second interview will be less than an hour, but will provide the participant as much time as needed to add new information or clarify information previously shared in-person.

RISKS: This study involves no physical risks. This study has the potential to involve psychological risks should emotions arise as participants share their story. There may also be other risks that we cannot predict. You can stop participating at any time. If you stop you will not lose any benefits.

BENEFITS TO TAKING PART IN THE STUDY? This study contributes to the research on careers in the student affairs profession by focusing on a specific, understudied population. Implications from this study may reveal key aspects of professional development applicable to individuals and organizations. There are no individual benefits to participating in this study beyond any benefits associated with personal reflection and sharing one's own story.

CONFIDENTIALITY: I will take the following steps to keep information about you confidential, and to protect it from unauthorized disclosure, tampering, or damage:

- Participants will be assigned a pseudonym to protect privacy. I will ensure confidentiality to the best of my ability in the final version of the dissertation and future publications.

- Steps will be taken to protect electronic data (pre-interview questionnaire, $\mathrm{CV}$, audio files of interviews). Files will be immediately transferred to a password protected computer on a secure network. The master list of participant names and assigned pseudonyms will be stored in a locked file cabinet.

- Only the principal investigator will have access to the above listed information. Should I have questions about my data analysis, I would only share hard copy interview transcripts with my doctoral supervisor. Original, electronic files will never be shared.

- Participants will have an opportunity to review the interview transcripts and identify any components that they think would be personally identifying. For my dissertation and future publications, I will do my best to use quotes that are not personally identifying, or I will alter quotes as needed to remove any specific names or institutions.

\section{YOUR RIGHTS AS A RESEARCH PARTICIPANT?}

Participation in this study is voluntary. You have the right not to participate at all or to leave the study at any time. Deciding not to participate or choosing to leave the study will not result in any penalty or loss of benefits to which you are entitled, and it will not harm your relationship with the principal investigator. Should you have questions or concerns about the study, you may contact Laura Page, Principal Investigator, at (573) 355-1866 or laurapage@mail.missouri.edu. You may also contact my doctoral advisor, Dr. Casandra Harper Morris at (573) 882-2818 or harpercas@missouri.edu. Should you have questions regarding your rights as a human subject research participant, you may contact the Institutional Review Board (IRB) at the University of Missouri at 573-882-3181 or irb@missouri.edu. 


\section{References}

AAU. (2017). Association of American Universities. Our members. Retrieved from https://www.aau.edu/

ACE. (1937). The student personnel point of view. Retrieved from http://www.myacpa.org/sites/default/files/student-personnel-point-of-view-1937.pdf

ACE (2017). American Council on Education. About. Retrieved from http://www.acenet.edu/about-ace/Pages/default.aspx

Ackerman, R. L. (Ed.). (2007). The mid-level manager in student affairs: Strategies for success. Washington, DC: National Association of Student Personnel Administrators (NASPA).

ACPA. (1996). The student learning imperative: Implications for student affairs. Washington, DC: Author. Retrieved from http://www.myacpa.org/sites/default/files/ACPA\%27s\%20Student\%20Learning\%20Impe rative.pdf

ACPA (2017). College Student Educators International. Mid-level community of practice. Retrieved from http://www.myacpa.org/mlcop

ACPA/NASPA. (2010a). Professional competency areas for student affairs practitioners. Washington, DC: ACPA and NASPA.

ACPA/NASPA. (2010b). Envisioning the future of student affairs: Final report of the task force on the future of student affairs. Retrieved from https://www.naspa.org/images/uploads/main/Task_Force_Student_Affairs_2010_Report. pdf

ACPA/NASPA. (2015). Professional competency areas for student affairs educators. Washington, D. C.: Authors. Retrieved from 
http://www.naspa.org/images/uploads/main/ACPA_NASPA_Professional_Competencies FINAL.pdf

Altbach, P. G. (2003). Globalization and the university: Myths and realities in an unequal world. Current Issues in Catholic Higher Education, 23(1), 5-26.

Andrews, M., Squire, C., \& Tamboukou, M. (Eds.). (2008). What is narrative research. London, England: Sage.

Aprianingsih, A. (2012). The Kaleidoscope Career Model and work family conflict: An exploration across career stages (Doctoral dissertation). Retrieved from ProQuest Dissertations and Theses. (UMI 3534993).

Bandura, A. (1977). Self-efficacy: Toward a unifying theory of behavioral change. Psychological review, 84(2), 191-215.

Banta, T. W. (2001). Assessing competence in higher education. In C. Palomba \& T. Banta (Eds.), Assessing student competence in accredited disciplines: Pioneering approaches to assessment in higher education (pp. 1-12). Sterling, VA: Stylus.

Barr, M. (2004). Foreward. In K. A. Renn \& C. Hughes (Eds.), Roads taken: Women in student affairs at mid-career (pp. xi-xvii). Sterling, VA: Stylus.

Bass, J. H. (2006). Vice president for student affairs and dean of students: Is it possible to do it all?. New Directions for Student Services, 2006(116), 45-52.

Baxter-Magolda, M. B. (1992). Knowing and reasoning in college: Gender-role patterns in students' intellectual development. San Francisco: Jossey-Bass.

Belch, H. A., \& Strange, C. C. (1995). Views from the bottleneck: Middle managers in student affairs. NASPA Journal, 32(3), 208-222. 
Belenky, M., Clinchy, B., Goldberger, N., \& Tarule, J. (1986). Women's Ways of Knowing: the development of self, voice, and mind. New York: Basic Books.

Bers, T. H. (2001). Measuring and reporting competencies. New Directions for Institutional Research, 110, 29-40.

Blackhurst, A., Brandt, J., \& Kalinowski, J. (1998). Effects of personal and work-related attributes on the organizational commitment and life satisfactions of women student affairs administrators. NASPA Journal, 35(2), 86-99.

Bloom (2002). From self to society: Reflections on the power of narrative inquiry. In S. B. Merriam (Ed.), Qualitative research in practice: Examples for discussion and analysis, pp. 310-313. San Francisco, CA: Jossey-Bass.

Braches, B. (2015). Gender in career transitions from corporate management to entrepreneurship (Doctoral dissertation). Retrieved from ProQuest Dissertations and Theses. (UMI 10180445).

Bresciani, M. L. (2008). Global competencies in student affairs/services professionals: A literature synthesis. College Student Journal, 42(3), 906.

Broughton, J. M. (1992). A critique of gender dualism in Gilligan's theory of moral development. In M. J. Larrabee (Ed.). An ethic of care: Feminist and interdisciplinary perspectives (p. 112-139). New York: Routledge.

Bruner, J. (1990). Culture and human development: A new look. Human Development, 33(6), 344-355.

Burbank, M. D., \& Kauchak, D. (2003). An alternative model for professional development: Investigations into effective collaboration. Teaching and Teacher Education, 19(5), 499514. 
Burkard, A. W., Cole, D. C., Ott, M., \& Stoflet, T. (2005). Entry-level competencies of new student affairs professionals: A delphi study. Journal of Student Affairs Research and Practice, 42(3), 545-571.

Burmeister, E., \& Aitken, L. M. (2012). Sample size: How many is enough? Australian Critical Care, 25(4), 271-274.

Butler, J. (1993). Bodies that matter. London, England: Routledge.

Carpenter, S., \& Haber-Curran, P. (2013). The role of research and scholarship in the professionalization of student affairs. Journal of Student Affairs in Africa, 1(1-2), 1-9.

Carraher, S. M., Crocitto, M. M., \& Sullivan, S. (2014). A kaleidoscope career perspective on faculty sabbaticals. Career Development International, 19(3), 295-313.

Chapman, K. J. (2014). The application of ACPA/NASPA professional competencies within student affairs divisions: A qualitative study (Unpublished doctoral dissertation). Texas Tech University, Lubbock, TX.

Cilente, K., Henning, G., Skinner Jackson, J., Kennedy, D., \& Sloan, T. (2006). Report on the New Professional Needs Study. Washington, DC: American College Personnel Association. Retrieved from http://www.myacpa.org/docs/npsfinalsurveyreportpdf

Clandinin, D. J., \& Connelly, F. M. (2000). Narrative inquiry: Experience and story in qualitative research. San Francisco, CA: Jossey-Bass.

Clifton, D. O., \& Anderson, E. (2001). StrengthsQuest: Discover and develop your strengths in academics, career, and beyond. (1st ed.). Washington, DC: Gallup Organization.

Cooper, J., Mitchell Jr, D., Eckerle, K., \& Martin, K. (2016). Addressing perceived skill deficiencies in student affairs graduate preparation programs. Journal of Student Affairs Research and Practice, 53(2), 107-117. 
Cortina, L. M. (2008). Unseen injustice: Incivility as modern discrimination in organizations. Academy of management review, 33(1), 55-75.

Craig, Cheryl J. (2009). Research in the midst of organized school reform: Versions of teacher community in tension. American Educational Research Journal, 46(2), 598-619. doi: $10.3102 / 0002831208330213$.

Creswell, J. W. (2013). Research design: Qualitative, quantitative, and mixed methods approaches. Thousand Oaks, CA: Sage.

Crotty, M. (2013). The foundations of social research: Meaning and perspective in the research process. London, England: Sage.

Cuyjet, M. J., Longwell-Grice, R., \& Molina, E. (2009). Perceptions of new student affairs professionals and their supervisors regarding the application of competencies learned in preparation programs. Journal of College Student Development, 50(1), 104-119.

Czarniawska, B. (2004). Narratives in social science research. London: Sage.

Daiute, C. (2014). Narrative inquiry: Dynamic narrating in life and research. Thousand Oaks, CA: Sage.

Davis, Kathy (1992). Toward a feminist rhetoric: The Gilligan debate revisited. Women's Studies International Forum, 15(2), 219-231.

Denzin, N. K., \& Lincoln, Y. S. (2011). The SAGE handbook of qualitative research. Thousand Oaks, CA: Sage.

Dickerson, A. M., Hoffman, J. L., Anan, B. P., Brown, K. F., Vong, L. K., Bresciani, M. J., Monzon, R., \& Oyler, J. (2011). A comparison of senior student affairs officer and student affairs preparatory program faculty expectations of entry-level professionals' competencies. Journal of Student Affairs Research and Practice, 48(4), 463-479. 
Dipboye, R. L., \& Halverson, S. K. (2004). Subtle (and not so subtle) discrimination in organizations. The dark side of organizational behavior, 16, 131-158.

Donaldson, J. F., \& Rosser, V. J. (2007). Midlevel leaders of continuing higher education:

Exploring their satisfaction, morale, and intentions to stay in or leave their positions. The Journal of Continuing Higher Education 55(1), 2-15.

Duffy, J. (2010). How women impacted the historical development of student affairs. College Student Affairs Journal, 28(2), 235-250.

Dungy, G. 2003. Organization and functions of student affairs. In S. R. Komives \& D. B. Woodard, Jr. (Eds.), Student services: A handbook for the profession (pp. 339-357). San Francisco: Jossey-Bass.

Eaton, P. W. (2016). The competency-based movement in student affairs: Implications for curriculum and professional development. Journal of College Student Development, 57(5), 573-589.

Elliott J (2005) Using narrative in social research: Qualitative and quantitative approaches. London: Sage.

Erlandson, D. A. (1993). Doing naturalistic inquiry: A guide to methods. Newbury Park, CA: Sage.

Ervin, S. (2015). Exploring the career needs of intercollegiate head coaches: A Kaleidoscope Career Perspective (Doctoral dissertation). Retrieved from ProQuest Dissertations and Theses. (UMI 3710154).

Fey, C. J., \& Carpenter, D. S. (1996). Mid-level student affairs administrators: Management skills and professional development needs. NASPA Journal, 33(3), 218-231. 
Forney, D. S. (1994). A profile of student affairs master's students: Characteristics, attitudes, and learning styles. Journal of College Student Development, 35(5), 337-345

Forret, M. L., Sullivan, S. E., \& Mainiero, L. A. (2010). Gender role differences in reactions to unemployment: Exploring psychological mobility and boundaryless careers. Journal of Organizational Behavior, 31(5), 647-666.

Freeman, S., Hagedorn, L. S., Goodchild, L. \& Wright, D. (Eds.). (2014). Advancing higher education as a field of study: In quest of doctoral degree guidelines - commemorating 120 years of excellence. Sterling, VA: Stylus.

Fusch, P. I., \& Ness, L. R. (2015). Are we there yet? Data saturation in qualitative research. The Qualitative Report, 20(9), 1408.

Gagnon, A. M. (2010). Resilient career narratives: An analysis of mid-career managers' longterm unemployment narratives (Doctoral dissertation). Retrieved from ProQuest Dissertations and Theses. (UMI 3397546).

Gansemer-Topf, A. M., \& Ryder, A. (2017). Competencies needed for entry-level student affairs work: Views from mid-level professionals. College Student Affairs Journal, 35(1), 40-54.

Gardner, S. K., \& Barnes, B. J. (2007). Graduate student involvement: Socialization for the professional role. Journal of College Student Development, 48(4), 369-387.

Gee, J. P. (2011). Discourse analysis: What makes it critical? In Rogers, R. (Ed.). An introduction to critical discourse analysis in education (pp. 23-45). New York, NY: Routledge.

Geertz, C. (1973). The interpretation of cultures. New York: Basic Books.

Gerda, J. J. (2006). Gathering together: A view of the earliest student affairs professional organizations. NASPA Journal, 43(4), 147-163. 
Gilligan, C. (1982). In a different voice. Cambridge, MA: Harvard University Press.

Goffman, E. (1959). The moral career of the mental patient. Psychiatry, 22(2), 123-142.

Golde, C. M. (1998). Beginning graduate school: Explaining first-year doctoral attrition. In M. S. Anderson (Ed.), The experience of being in graduate school: An exploration (pp. 55-64). San Francisco: Jossey-Bass.

Golde, C. M. (2000). Should I stay or should I go? Student descriptions of the doctoral attrition process. The Review in Higher Education, 23(2), 199-227.

Golde, C. M., \& Dore, T. (2001). At cross purposes: What the experiences of today's doctoral students reveal about doctoral education. Philadelphia: Pew Charitable Trusts.

Grube, S. A., Cedarholm, K., Jones, C., \& Dunn, M. (2005). Master's student life: The balance between student and professional. College Student Affairs Journal, 24(2), 152-161.

Guba, E. G., \& Lincoln, Y. S. (1994). Competing paradigms in qualitative research. In N. K. Denzin \& Y. S. Lincoln (Eds.), The handbook of qualitative research (pp. 105-117). Thousand Oaks, CA: Sage

Guthrie, V. L., Woods, E., Cusker, C., \& Gregory, M. (2005). A portrait of balance: Personal and professional balance among student affairs educators. College Student Affairs Journal, 24(2), 110.

Haessig, C. \& LaPotin, A. (2000). Outcomes assessment for dietetics educators. Chicago, IL: Commission of Accreditation for Dietetics Education, The American Dietetics Association.

Hall, R. M., \& Sandier, B. (1982). The classroom climate: A chilly one for women? Project on the Status of Women. Washington, DC: Association of American Colleges and Universities. 
Hart, J. (2016). Dissecting a gendered organization: Implications for career trajectories for midcareer women in STEM. Journal of Higher Education, 87(5), 605-634.

Herdlein, R. J. (2004). Survey of chief student affairs officers regarding relevance of graduate preparation of new professionals. Journal of Student Affairs Research and Practice, 42(1), 51-71.

Herdlein, R., Riefler, L., \& Mrowka, K. (2013). An integrative literature review of student affairs competencies: A meta-analysis. Journal of Student Affairs Research and Practice, 50(3), 250-269.

Higgins M. C., \& Kram K. E. (2001). Reconceptualizing mentoring at work: A developmental network perspective. Academy of Management Review, 26, 264-288.

Hirsch, M. S. \& Berman-Hills, L. (2004). Strategic women. In K. A. Renn \& C. Hughes (Eds.), Roads taken: Women in student affairs at mid-career (pp. 189-197). Sterling, VA: Stylus.

Hoffman, J. L., \& Bresciani, M. J. (2012). Identifying what student affairs professionals value: An analysis of professional competencies listed in job descriptions. Research and Practice in Assessment, 7(1), 26-40.

Horton, S. S. (2004). I've made it: What now? In K. A. Renn \& C. Hughes (Eds.), Roads taken: Women in student affairs at mid-career (pp. 153-160). Sterling, VA: Stylus.

Howard-Hamilton, M. F. (2004). Considering the doctorate: Introduction. In K. A. Renn \& C. Hughes (Eds.), Roads taken: Women in student affairs at mid-career (pp. 3-11). Sterling, VA: Stylus.

Hughes (2004). "I've arrived”: It's the journey, not the destination: Introduction. In K. A. Renn \& C. Hughes (Eds.), Roads taken: Women in student affairs at mid-career (pp. 135-142). Sterling, VA: Stylus. 
Hunter, S. V. (2010). Analysing and representing narrative data: The long and winding road. Current Narratives, 1(2), 44-54.

Janosik, S. M., Cooper, D. L., Saunders, S. A., \& Hirt, J. B. (2014). Learning through supervised practice in student affairs. New York: Routledge.

Jones, J. M. (2016). Gallup-Lumina Foundation. Americans still say postsecondary education very important. Retrieved from http://www.gallup.com/poll/190580/americans-saypostsecondary-education-important.aspx

Jones, S. R. (2004). A degree of significance: Personal and professional consideration in pursuing a Ph.D. In K. A. Renn \& C. Hughes (Eds.), Roads taken: Women in student affairs at mid-career (pp. 13-21). Sterling, VA: Stylus.

Jones, S. R., \& Komives, S. R. (2001). Contemporary issues of women as senior student affairs officers In J. Nidiffer \& C. T. Bashaw (Eds.), Women administrators in higher education (pp. 231-248). Albany: SUNY Press.

Josselson, R. (2011). Narrative research. In F. J. Wertz, K. Charmaz, L. M. McMullen, R. Josselson, R. Anderson, \& E. McSpadden (Eds.), Five ways of doing qualitative analysis (pp. 224-242). New York, NY: Guilford.

Joyce-Brady, J. (2004). How did I arrive here? A journey with family and student affairs. In K. A. Renn \& C. Hughes (Eds.), Roads taken: Women in student affairs at mid-career (pp. 113-122). Sterling, VA: Stylus.

June, A. W. (2007). Presidents: Same look, Different decade. Chronicle of Higher Education, 53(24), A33. 
Keeling, R. P. (Ed.). (2004). Learning reconsidered: A campus-wide focus on the student experience. Washington, DC: National Association of Student Personnel Administrators and American College Personnel Association.

Keeling, R. P. (2006). Learning reconsidered 2: Implementing a campus-wide focus on the student experience. Washington, DC: American College Personnel Association, Association of College and University Housing Officers International, Association of College Unions-International, National Academic Advising Association, National Association for Campus Activities, National Association of Student Personnel Administrators, and National Intramural Recreational Sports Association.

Kerber, L. K. (1986). Some cautionary words for historians. Signs: Journal of Women in Culture and Society, 11(2), 304-310.

Kezar, A. J., Carducci, R., \& Contreras-McGavin, M. (2006). Rethinking the " $L$ " word in higher education: The revolution in research on leadership. ASHE Higher Education Report, Vol. 31, No. 6. San Francisco: Jossey-Bass.

Kolb, D. (1984). Experiential learning as the science of learning and development. Eaglewood Cliffs, NJ: Prentice Hall

Kretovics, M. (2002). Entry-level competencies: What student affairs administrators consider when screening candidates. Journal of College Student Development, 43(6), 912-920.

Kuk, L., \& Banning, J. H. (2016). Student Affairs Leadership: Defining the Role Through an Ecological Framework. Sterling, VA: Stylus.

Kuk, L., Cobb, B., \& Forrest, C. S. (2007). Perceptions of competencies of entry-level practitioners in student affairs. Journal of Student Affairs Research and Practice, 44(4), 1061-1088. 
Lent, R. W., Brown, S. D., \& Hackett, G. (1994). Toward a unifying social cognitive theory of career and academic interest, choice, and performance. Journal of vocational behavior, 45(1), 79-122.

Levinson, S. C. (2000). Presumptive meanings: The theory of generalized conversational implicature. Cambridge, MA: MIT Press.

Liamputtong, P., \& Ezzy, D. (2005). In depth interviews. In P. Liamputtong \& Ezzy (Eds.), Qualitative research methods (2nd ed., pp. 54-73). Victoria, Australia: Oxford.

Lilley, K., Barker, M., \& Harris, N. (2017) The global citizen conceptualized: Accommodating ambiguity. Journal of Studies in International Education, 21(2), 6-21.

Lincoln, Y.S., \& Guba, E.G. (1985). Naturalistic inquiry. Newbury Park, CA: Sage.

Lincoln Y., Guba E. (2002). The only generalization is: There is no generalization. In R. Gomm, M. Hammersley, \& P. Foster (Eds.), Case study method (pp. 27-44). London: Sage.

Lofland, J. \& Lofland, L. H. (1995). Analyzing social settings: A guide to qualitative observation and analysis ( $3^{\text {rd }}$ ed.). Belmont, CA: Wadsworth.

Love, P. (2003). ACPA. Considering a career in student affairs. Retrieved from http://www.myacpa.org/considering-career-student-affairs

Love, P. G. \& Guthrie, V. L. (Eds.). (1999). Understanding and applying cognitive development theory. New Directions for Student Services, No. 88. San Francisco: Jossey-Bass.

Mainiero, L. A., \& Sullivan, S. E. (2005). Kaleidoscope careers: An alternate explanation for the "opt-out "revolution. The Academy of Management Executive, 19(1), 106-123.

Mainiero, L. A. \& Sullivan, S. E. (2006). The opt-out revolt: Why people are leaving companies to create kaleidoscope careers. Mountain View, CA: Davis-Black. 
Mannay, D. (2015). Visual, narrative and creative research methods: Application, reflection and ethics. New York: Routledge.

Manning, K., Kinzie, J., \& Schuh, J. H. (2015). One size does not fit all: Traditional and innovative models of student affairs practice. New York: Routledge.

Marshall, S. (2004). Motherhood and student affairs: The skillful art of managing work and family: Introduction. In K. A. Renn \& C. Hughes (Eds.), Roads taken: Women in student affairs at mid-career (pp. 89-100). Sterling, VA: Stylus.

Mather, P. C., Smith, K. J., \& Skipper, S. (2010). A matter of competence: A national study of the preparation of new student affairs professionals. International Journal of Educational Leadership Preparations, 5(4), 1-15.

Maurer, C. \& Sheets, T. E. (1998). Encyclopedia of associations. Detroit: Gale Research.

McNair, D. E., Miguel, K, Sobers-Young, S. T., Bechtel, M., Jacobson, S. (2013). Women in student affairs: Past, present, and future leaders. NASPA Journal About Women in Higher Education. 6(2), 231-250.

Merriam, S. B. (2009). Qualitative research: A guide to design and implementation: Revised and expanded from qualitative research and case study applications in education. San Francisco: Jossey-Bass.

Mills, D. B. (2007). Middle managers: Roles and responsibilities spanning the student affairs career. In G. S. McClellan, J. Stringer, \& Associates (Eds.), The handbook of student affairs administration, (pp. 355-370). Washington, DC: NASPA.

Nadal, K. L., Meterko, V., Vargas, V. M., \& Wideman, M. (2016). Empowering women leaders to rise above microaggressions. In M. A. Paludi (Ed.). Why congress needs women: Bringing sanity to the house and senate. (p. 121-136). Santa Barbara: Praeger 
Nash, R. J., \& Bradley, D. L. (2012). The writer is at the center of the scholarship: Partnering me-search and research. About Campus, 17(1), 2-11. doi: 10.1002/abc.21067

NASPA (2017a) Student Affairs Administrators in Higher Education. Mid-Level Institute at the Joint IV-WE Conference. Retrieved from https://www.naspa.org/events/MLIinSTL

NASPA (2017b) Student Affairs Administrators in Higher Education. NASPA Journal About Women in Higher Education. Retrieved from https://www.naspa.org/publications/journals/naspa-journal-about-women-in-highereducation

NASPA (2017c). Student Affairs Administrators in Higher Education. Graduate Program Directory. Retrieved from https://www.naspa.org/careers/graduate/graduate-programdirectory

National Center for Education Statistics (2016). College Navigator. Retrieved from https://nces.ed.gov/collegenavigator/

Nelson, K. (1989). Narratives from the crib. Cambridge, MA: Harvard University Press.

Nuss, E.M. (2003). The development of student affairs. In S. R. Komives \& D. B. Woodard, Jr. (Eds.), Student services: A handbook for the profession (4th ed.), (pp. 65-88). San Francisco: Jossey-Bass.

Nyquist, J. D., Manning, L., Wulff, D. H., Austin, A. E., Sprague, J., Fraser, P. K., Calcagno, C., \& Woodford, B. (1999). On the road to becoming a professor: The graduate student experience. Change, 31(3), 18-27.

Olyha, G. P. (2004). New York to New England and seas in between: On the way to twenty years in one place. In K. A. Renn \& C. Hughes (Eds.), Roads taken: Women in student affairs at mid-career (pp. 1143-152). Sterling, VA: Stylus. 
O’Neil, D., Bilimoria, D., \& Saatcioglu, A. (2003). Women's ways of instituting careers: A typology of women's career development. Paper presented at the Academy of Management, Best Conference Paper Careers Division, Seattle, WA.

Ortiz, A. M. (2004). Arriving at tenure. In K. A. Renn \& C. Hughes (Eds.), Roads taken: Women in student affairs at mid-career (pp. 161-170). Sterling, VA: Stylus.

Ott, M., Baca, E., Cisneros, J., \& Bates, E. (2015). A competency-based approach to the master's degree preparation of higher education professionals. Journal of Case Studies in Accreditation and Assessment (4). Retrieved from http://www.aabri.com/manuscripts/141948.pdf

Otter S (1995). Higher level NVQs/SVQs: Their possible implications for higher education, Competence and Assessment, 29(6), 33-38

Patton, M. Q. (1980). Qualitative evaluation methods. Beverly Hills, CA: Sage.

Patton, M. Q. (2002): Qualitative research and evaluation methods. Thousand Oaks, CA: Sage.

Perez, R. J. (2014). Exploring the cognitive dimensions of professional socialization in student affairs (Doctoral dissertation). Retrieved from Deep Blue, University of Michigan Library http://deepblue.lib.umich.edu/handle/2027.42/108829

Phoenix, A. (2008). Analysing narrative contexts. In M. Andrews, C. Squire, M. Tamboukou (Eds.) Doing narrative research (pp. 64-77). London: Sage.

Pinnegar, S., \& Daynes, G. (2007). Locating narrative inquiry historically. In D. J. Clandinin (Ed.), Handbook of narrative inquiry (pp. 3-34). London: Sage.

Polkinghorne, D. E. (2005). Language and meaning: Data collection in qualitative research. Journal of Counseling Psychology, 52(2), 137-145. 
Powell, G. N., \& Mainiero, L. A. (1992). Cross-currents in the river of time: Conceptualizing the complexities of women's careers. Journal of Management, 18, 215-237.

Qualtrics, L.L.C. (2017). About us. Retrieved from https://www.qualtrics.com/about/

Reason, R. D., \& Kimball, E. W. (2012). A new theory-to-practice model for student affairs: Integrating scholarship, context, and reflection. Journal of Student Affairs Research and Practice, 49(4), 359-376.

Renn, K. A., \& Hodges, J. (2007). The first year on the job: Experiences of new professionals in student affairs. Journal of Student Affairs Research and Practice, 44(2), 604-628.

Renn, K. A., \& Hughes, C. (2004). Roads taken: Women in student affairs at mid-career. Sterling, VA: Stylus.

Renn, K. A., \& Jessup-Anger, E. R. (2008). Preparing new professionals: Lessons for graduate preparation programs from the national study of new professionals in student affairs. Journal of College Student Development, 49(4), 319-335.

Rhatigan, J.J. (2000). The handbook of student affairs administration (2nd ed.). San Francisco, CA: Jossey-Bass.

Rickard, S. T. (1985). Career pathways of chief student affairs officers: Making room at the top for females and minorities. NASPA Journal, 22(4), 52-60.

Riessman, C. K. (1993). Narrative analysis. Newbury Park, CA: Sage.

Riessman, C. K. (2008). Narrative methods for the human sciences. Thousand Oaks, CA: Sage. Rosser, V. J. (2000). Midlevel administrators: What we know. In L. Johnsrud and V. Rosser (Eds.), Understanding the work and career paths of midlevel administrators, New Directions for Higher Education, 111. (pp. 5-13). San Francisco: Jossey-Bass. 
Rosser, V. J., \& Javinar, J. M. (2003). Midlevel student affairs leaders' intentions to leave:

Examining the quality of their professional and institutional work life. Journal of College Student Development, 44(6), 813-830.

Sallee, M. W. (2011). Performing masculinity: Considering gender in doctoral student socialization. The Journal of Higher Education, 82(2), 187-216

Salter, D. W. (2003). Exploring the "chilly classroom" phenomenon as interactions between psychological and environmental types. Journal of College Student Development, 44(1), $110-121$.

Sandeen, A. (1991). The chief student affairs officer: Leader, manager, mediator, educator. San Francisco, CA: Jossey-Bass.

Sandeen, A. (2004). Educating the whole student: The growing academic importance of student affairs. Change: The Magazine of Higher Learning, 36(3), 28-33.

Sandeen, A., \& Barr, M. J. (2014). Critical issues for student affairs: Challenges and opportunities. Hoboken, NJ: John Wiley \& Sons.

Sandler, B. R. (1986). The campus climate revisited: Chilly for women faculty, administrators, and graduate students [final report]. Washington, DC: Fund for the Improvement of Postsecondary Education.

Schaufeli, W. B., \& Enzmann, D. (1998). The burnout companion to study and research: A critical analysis. London, UK: Taylor and Francis.

Schensul, J. J., LeCompte, M. D., Trotter II, R. T., Cromley, E. K., \& Singer, M. (1999). Mapping social networks, Spacial data, and hidden populations. Ethnographers toolkit. Walnut Creek, CA: Altamira Press. 
Schulz, S. A., Lee, J. J., Cantwell, B. J., McClellan, G., \& Woodard, D. (2007). Moving toward a global community: An analysis of the internationalization of student affairs graduate preparation programs. NASPA Journal, 44(3), 610-632.

Schwartz, R. A., \& Bryan, W. A. (1998). Strategies for staff development: Personal and professional education in the 21st Century. San Francisco, CA: Jossey-Bass.

Scott, J. E. (2000). Creating effective staff development programs. In M. J. Barr \& M. K. Desler, (Eds.), The handbook of student affairs administration, (pp. 477-491). San Francisco, CA: Jossey-Bass.

Simmons, J. (2012). The Kaleidoscope Career Model: An investigation of authenticity, balance, and challenge and their relationship with networking behavior (Doctoral dissertation). Retrieved from ProQuest Dissertation and Theses. (UMI 3534994).

Spence, D. P. (1982). Narrative truth and theoretical truth. The psychoanalytic quarterly, 51(1), 43.

Sponsler, B., \& Wesaw, A. (2014). Vice president for student affairs census. NASPA Research and Policy Institute. Washington, DC: NASPA. Retrieved from http://census.naspa.org/

Squire, C., Andrews, M., \& Tamboukou, M. (2008). Doing narrative research. Los Angeles, CA: Sage.

Sriram, R. (2014). The development, validity, and reliability of a psychometric instrument measuring competencies in student affairs. Journal of Student Affairs Research and Practice, 51(4), 349-363.

Stake, R. E. (2010). Qualitative research: Studying how things work. New York: Guilford Press. 
Sullivan, S. E., Forret, M. L., Carraher, S. M., \& Mainiero, L. A. (2009). Using the Kaleidoscope Career Model to examine generational differences in work attitudes. Career Development International, 14(3), 284-302.

Sullivan, S. E., Forret, M. L., Mainiero, L. A., \& Terjesen, S. (2007). What motivates entrepreneurs? An exploratory study of the kaleidoscope career model and entrepreneurship. Journal of Applied Management and Entrepreneurship, 12(4), 4.

Sullivan, S. E., \& Mainiero, L. A. (2007). Kaleidoscope careers: Benchmarking ideas for fostering family-friendly workplaces. Organizational Dynamics, 36(1).

Sullivan, S. E., \& Mainiero, L. (2007). Women's kaleidoscope careers: A new framework for examining women's stress across the lifespan. In P. L. Perrew \& D. C. Ganster (Eds.), Exploring the work and nonwork interface, research in occupational stress: Volume 6 (pp. 205-238). Greenwich, CT: JAI.

Sullivan, S. E., \& Mainiero, L. A. (2008). Using the kaleidoscope career model to understand the changing patterns of women's careers: Designing HRD programs that attract and retain women. Advances in Developing Human Resources, 10(1), 32-49.

Tajlili, M. H. (2015). The future work-life balance attitudes scale: Creation of an instrument to assess millennial college women's attitudes on work-life balance (Doctoral dissertation). Retrieved from ProQuest Dissertations and Theses. (UMI 3710701)

Taub, D. J., \& McEwen, M. K. (2006). Decision to enter the profession of student affairs. Journal of College Student Development, 47(2), 206-216.

The Carnegie Classification of Institutions of Higher Education. (n.d.). About Carnegie Classification. Retrieved from http://carnegieclassifications.iu.edu/. 
Thelin, J. R. (2011). A history of American higher education. Baltimore, MD: Johns Hopkins University Press.

Theobald, M. (1999). Teachers, memory and oral history. In K. Weiler and S. Middleton (Eds.) Telling women's lives: Narrative inquiries in the history of women's education (9-24). Buckingham, England: Open University Press.

Thomas K. M., Bierema L., Landau H. (2004). Advancing women's leadership in academe: New directions for research and HRD practice. Equal Opportunities International, 27(7/8), 6277.

Tull, A. (2006). Synergistic supervision, job satisfaction, and intention to turnover of new professionals in student affairs. Journal of College Student Development, 47(4), 465-480.

Tull, A., \& Kuk, L. (2012). New realities in the management of student affairs: Emerging specialist roles and structures for changing times. Sterling, VA: Stylus.

U. S. Department of Education (n.d.). Federal TRIO programs. Retrieved from https://www2.ed.gov/about/offices/list/ope/trio/index.html

U. S. Department of Labor (2014). Bureau of Labor Statistics. Retrieved from https://www.bls.gov/ooh/management/postsecondary-education-administrators.htm

Voorhees, R. A. (2001). Competency-based learning models: A necessary future. New directions for institutional research, 2001(110), 5-13.

Weidman, J. C., Twale, D. J., and Stein, E. L. (2001). Socialization of graduate and professional students in higher education: A perilous passage? ASHE-ERIC Higher Education Report, 28. Jossey-Bass, San Francisco.

West, C., \& Zimmerman, D. H. (1987). Doing gender. Gender \& society, 1(2), 125-151. 
Wheeler, P., \& Haertel, G. D. (1993). Resource handbook on performance assessment and measurement: A tool for students, practitioners, and policymakers. Berkeley, CA: Owl Press.

Williamson, E. G., Blaesser, W. W., Bragdon, H. D., Carlson, W. S., Cowley, W. H., Feder, D. D., Fisk, H. G., Kirkpatrick, F. H., Lloyd-Jones, E., McConnell, T. R., Merriam, T. W., \& Shank, D. J. (1949). The student personnel point of view. Retrieved from https://www.naspa.org/images/uploads/main/Student_Personnel_Point_of_View_1949.pd $\mathrm{f}$

Wilson, M. E., Liddell, D. L., Hirschy, A. S., \& Pasquesi, K. (2016). Professional identity, career commitment, and career entrenchment of midlevel student affairs professionals. Journal of College Student Development, 57(5), 557-572.

Wodak, R., \& Meyer, M. (Eds.). (2015). Methods of critical discourse studies (3rd ed.). Los Angeles, CA: Sage.

Wolcott, H. F. (2010). Ethnography lessons: A primer. Walnut Creek, CA: Left Coast Press. Wong, J. M. (2004). My life A.D. (after divorce). In K. A. Renn \& C. Hughes (Eds.), Roads taken: Women in student affairs at mid-career (pp. 35-40). Sterling, VA: Stylus.

Yakaboski, T., \& Donahoo, S. (2010). In (re)search of women in student affairs administration. In P. A. Pasque \& S. E. Nicholson (Eds.), Women in higher education and student affairs: Research and practice from feminist perspectives. Sterling, VA: Stylus.

Young, R. B. (Ed.). (1990). The invisible leaders: Student affairs mid-managers. Washington, DC: National Association of Student Personnel Administrators. 
Yourston, D. (2016). An investigation into the role of generational differences in the career types, progression and success of British managers (Doctoral dissertation). Retrieved from ProQuest Dissertations and Theses. (UMI 10594759) 


\section{VITA}

Laura Alexander Page is a native of the North Carolina mountains with deep roots in both of the Carolinas. Always committed to the pursuit of education, her undergraduate student experience influenced the paths she would take both personally and professionally. Laura's keen interest in people and communities led her to pursue a Bachelor of Science in Sociology with a Minor in Guidance Counseling at Western Carolina University, and her involvement as a student leader there connected her to the student affairs field as a future career. She received formal education in student affairs at Clemson University by completing a Master of Education in Counseling and Guidance Services with an emphasis in Student Affairs Administration, while also receiving practical training through graduate assistantships, internships, practicums, and a study abroad experience where she worked in student services at the Exmouth campus of the University of Plymouth in England. While at Clemson, she met her husband, also a student affairs educator, and together they worked at Clemson, the University of South Carolina, and the University of Missouri. After twelve years in a variety of professional roles in enrollment management, student life, and fundraising and external relations, teaching first year seminar courses, and professional involvement both regionally and nationally, Laura returned to the classroom as a full-time doctoral student at the University of Missouri. Through balancing advanced graduate work with a family and continued campus and professional involvement, Laura completed a Ph.D. in Educational Leadership and Policy Analysis with a concentration in Higher Education Administration and a Minor in College Teaching (to be conferred in August 2017). She currently lives in Columbia, Missouri, with her husband and three young daughters. 